\title{
Foundations of finite element methods for wave equations of Maxwell type*
}

\author{
Snorre H. Christiansen ${ }^{\dagger \ddagger}$
}

\begin{abstract}
The first part of the paper is an overview of the theory of approximation of wave equations by Galerkin methods. It treats convergence theory for linear second order evolution equations and includes studies of consistency and eigenvalue approximation. We emphasize differential operators, such as the curl, which have large kernels and use $\mathrm{L}^{2}$ stable interpolators preserving them. The second part is devoted to a framework for the construction of finite element spaces of differential forms on cellular complexes. Material on homological and tensor algebra as well as differential and discrete geometry is included. Whitney forms, their duals, their high order versions, their tensor products and their $h p$-versions all fit.
\end{abstract}

\section{Introduction}

The Yee scheme [62] is a very efficient finite difference scheme for simulating the initial/boundary value problem for Maxwell's equations. It is used in many industrial codes for problems ranging from antenna design, to electromagnetic compatibility and to medical imaging. However it is only second order accurate, it treats boundary conditions in a rough way (stair-casing) and it is not clear how it should be formulated for anisotropic materials. All three problems can be addressed in the finite element framework. For electromagnetics, mixed finite elements [53] [49] [50] [15], have been found to give the best results.

Finite elements come with a natural way of deriving error estimates. Stability of the method is linked to energy conservation, which is almost automatic for variational Galerkin methods. Charge conservation is also ensured weakly by these methods. Through stability, the convergence of the method is reduced to the problem of estimating errors of best approximation on the finite element space. They are usually obtained from interpolation operators.

\footnotetext{
*to appear in "Applied Wave Mathematics - Selected Topics in Solids, Fluids, and Mathematical Methods" edited by Ewald Quak and Tarmo Soomere, Springer Verlag.

$\dagger$ CMA, University of Oslo, PO Box 1053 Blindern, NO-0316 Oslo, Norway.

$\ddagger$ This work, conducted as part of the award "Numerical analysis and simulations of geometric wave equations" made under the European Heads of Research Councils and European Science Foundation EURYI (European Young Investigator) Awards scheme, was supported by funds from the Participating Organizations of EURYI and the EC Sixth Framework Program.
} 
The main drawback of the finite element method is that the mass matrix is not diagonal leading to (linearly) implicit methods. In some circumstances a procedure known as mass-lumping solves this problem. In particular one can recover the Yee scheme in this way.

One of the reasons for the success of the Yee scheme and mixed finite elements is that they respect the geometry of Maxwell's equations [12] [59]. The matrix of the differential operators grad, curl and div in the standard basis of lowest order mixed finite elements are the incidence matrices of algebraic topology. Mixed finite elements are equipped with interpolation operators which behave well with respect to these differential operators: they form commuting diagrams linking continuous and discrete complexes of spaces.

The present paper is divided in two parts. The first part is devoted to convergence analysis of the Galerkin method for waves assuming just some basic properties of the discretization spaces. We start with the variational (weak) formulation of wave equations, adopting a general framework that covers scalar waves as well as electromagnetic waves. Convergence is proved using energy estimates, with an emphasis on rough data. Then we study consistency, which is the theoretical foundation for mass-lumping. Finally we treat the eigenvalue problem, basing the theory on the existence of $\mathrm{L}^{2}$ stable interpolators vanishing on the kernel of the bilinear form.

The second part is devoted to the construction of finite element spaces. We first review some tools from algebraic topology, such as the long exact sequence and the five lemma, and tensor algebra including the Kunneth theorem. Then we introduce differential forms which enables one to treat the grad, curl and div operators in a uniform manner, as well as boundary conditions for various types of fields. Finally we develop a framework for the construction and study of finite element spaces of differential forms on cellular complexes as in [23]. The dual of a simplicial complex is not a simplicial complex, nor is the product of two simplicial complexes. However duals and products of cellular complexes are again cellular complexes, so that we gain some flexibility from this level of generality. Tensor products of high order Whitney forms, which correspond to mixed finite elements on products of simplexes, fit the framework as well as the dual finite elements introduced in [18]. Even $h p$-finite elements [35] and projection based interpolation are accommodated.

A number of surveys on these topics have been published in the last years, in particular [40], [42], [48] and [5]. The algebra can be found in textbooks but is most often mixed with considerations on homology (e.g. singular) and modules. The claim to originality of the present study, if any, is the emphasis on rough data through $\mathrm{L}^{2}$ stable interpolation and the introduction of general constructions on cellular complexes.

\section{Analysis of the finite element method for waves}

\subsection{Linear wave equations}

When dealing with a time dependent quantity $u$ we denote its first and second time derivatives by $\dot{u}$ and $\ddot{u}$ respectively. Let $U$ be an open bounded domain in $\mathbb{R}^{n}$. The linear wave equation for a function $u: \mathbb{R} \times U \rightarrow \mathbb{R}$, 
with forcing term $f: \mathbb{R} \times U \rightarrow \mathbb{R}$ is:

$$
\ddot{u}=\Delta u+f,
$$

where $\Delta$ is the Laplace operator on $U$. We will consider homogeneous Dirichlet boundary conditions, namely $u(t, x)=0$ for all $t \in \mathbb{R}$ and $x \in$ $\partial U$. Initial conditions $u_{0}$ and $\dot{u}_{0}$ are supplied for $u(0)$ and $\dot{u}(0)$. Our point of view is that $u_{0}, \dot{u}_{0}$ and $f$ constitute the data of the problem whereas $u$ is the unknown function we want to compute a reasonable approximation of. It will be necessary to interpret both the wave equation and initialvalue/boundary conditions in a weak sense (not pointwise).

The $\mathrm{L}^{2}$ scalar product is denoted $\langle\cdot, \cdot\rangle$ both on functions and vectorfields on $U$ (assuming the standard scalar product on vectors in $\mathbb{R}^{n}$ ). Recall that for smooth functions $u, v$ satisfying the Dirichlet boundary condition we have:

$$
\langle\Delta u, v\rangle=-\langle\operatorname{grad} u, \operatorname{grad} v\rangle .
$$

The energy of $u$ at time $t$ is:

$$
\mathcal{E}(u)(t)=1 / 2\langle\dot{u}(t), \dot{u}(t)\rangle+1 / 2\langle\operatorname{grad} u(t), \operatorname{grad} u(t)\rangle .
$$

When the forcing term $f$ is 0 , energy is conserved, for smooth enough solutions. We will always deal with finite energy solutions, that is, functions for which $\mathcal{E}(u)$ is essentially bounded.

The weak formulation of (1) in space, is to require that $u(t) \in \mathrm{H}_{0}^{1}(U)$ and for all test-functions $u^{\prime}$ :

$$
\left\langle\ddot{u}(t), u^{\prime}\right\rangle=-\left\langle\operatorname{grad} u(t), \operatorname{grad} u^{\prime}\right\rangle+\left\langle f(t), u^{\prime}\right\rangle .
$$

It remains to give a meaning to $\ddot{u}(t)$ and the initial conditions. We solve: given $u_{0} \in \mathrm{H}_{0}^{1}(U)$ and $\dot{u}_{0} \in \mathrm{L}^{2}(U)$ find:

$$
u \in C\left([0, T] ; \mathrm{H}_{0}^{1}(U)\right) \cap C^{1}\left([0, T] ; \mathrm{L}^{2}(U)\right),
$$

satisfying the initial conditions and such that (2) holds in some sense. For instance we can require that for all $u^{\prime} \in C_{0}^{2}\left(\left[0, T\left[; \mathrm{H}_{0}^{1}(U)\right)\right.\right.$ :

$$
\begin{aligned}
\int\left\langle u(t), \ddot{u}^{\prime}(t)\right\rangle \mathrm{d} t+ & \left\langle\dot{u}_{0}, u^{\prime}(0)\right\rangle-\left\langle u_{0}, \dot{u}^{\prime}(0)\right\rangle= \\
& -\int\left\langle\operatorname{grad} u(t), \operatorname{grad} u^{\prime}(t)\right\rangle \mathrm{d} t+\int\left\langle f(t), u^{\prime}(t)\right\rangle \mathrm{d} t .
\end{aligned}
$$

When $f \in \mathrm{L}^{1}\left([0, T] ; \mathrm{L}^{2}(U)\right)$, this problem has a unique solution. This can be proved by the Galerkin method, to which we now turn our attention.

More generally we consider the following situation. Let $H$ be a Hilbert space with scalar product $\langle\cdot, \cdot\rangle$ and norm $|\cdot|$. Suppose $H$ contains a Hilbert space $X$ which is dense and such that the inclusion $X \rightarrow H$ is continuous. The wave equation (1) corresponds to the choices $H=\mathrm{L}^{2}(U)$ equipped with the standard $\mathrm{L}^{2}$ product and $X=\mathrm{H}_{0}^{1}(U)$. Suppose $a$ is a continuous symmetric bilinear form on $X$ such that for all $u \in X$ :

$$
a(u, u) \geq 0,
$$

and:

$$
\langle\cdot, \cdot\rangle+a(\cdot, \cdot) \text { is coercive on } X
$$


The scalar wave equation corresponds to the choice:

$$
a(u, v)=\int \operatorname{grad} u \cdot \operatorname{grad} v .
$$

Notice that we allow $a$ to have a possibly infinite-dimensional kernel. This will be the case when we apply the abstract setting to Maxwell's equations. As a compatible norm on $X$ we can take the one defined by:

$$
\|u\|^{2}=\langle u, u\rangle+a(u, u) .
$$

We are interested in the initial value problem for the second order evolution equation:

$$
\forall u^{\prime} \in X \quad\left\langle\ddot{u}, u^{\prime}\right\rangle=-a\left(u, u^{\prime}\right)+\left\langle f, u^{\prime}\right\rangle,
$$

Given $u_{0} \in X$ and $\dot{u}_{0} \in H$ as well as $f \in \mathrm{L}^{1}([0, T] ; H)$ we look for $u \in C([0, T] ; X) \cap C^{1}([0, T] ; H)$ such that for all $u^{\prime} \in C_{0}^{2}([0, T[; X)$ :

$$
\begin{aligned}
\int\left\langle u(t), \ddot{u}^{\prime}(t)\right\rangle \mathrm{d} t+ & \left\langle\dot{u}_{0}, u^{\prime}(0)\right\rangle-\left\langle u_{0}, \dot{u}^{\prime}(0)\right\rangle= \\
& -\int a\left(u(t), u^{\prime}(t)\right) \mathrm{d} t+\int\left\langle f(t), u^{\prime}(t)\right\rangle \mathrm{d} t .
\end{aligned}
$$

We call this the abstract wave equation. The energy of a function $u$ is defined by :

$$
\mathcal{E}(u)(t)=1 / 2\langle\dot{u}(t), \dot{u}(t)\rangle+1 / 2 a(u(t), u(t)) .
$$

This level of generality includes wave equations in media with variable, possibly discontinuous, coefficients and also the Maxwell's equations. We sketch this last point.

We work with an open domain $U$ in $\mathbb{R}^{3}$, filled with vacuum. The unknowns are two time-dependent vectorfields $E$ and $B$ on $U$ called the electric and magnetic field. The first pair of Maxwell's equations is:

$$
\begin{aligned}
\dot{B} & =-\operatorname{curl} E, \\
\operatorname{div} B & =0 .
\end{aligned}
$$

The second pair is:

$$
\begin{aligned}
\dot{E} & =\operatorname{curl} B+J, \\
\operatorname{div} E & =Q .
\end{aligned}
$$

The right hand side are a time-dependent scalar field $Q$ called charge and a time-dependent vector field $J$ called current.

Notice that we must have conservation of charge:

$$
\dot{Q}=\operatorname{div} J
$$

The first pair of equations guarantees the existence (at least on simply connected domains) of a magnetic potential $A$ which is a time dependent vectorfield on $U$ such that:

$$
\begin{aligned}
& E=-\dot{A}, \\
& B=\operatorname{curl} A .
\end{aligned}
$$


The first pair of equations is then automatically satisfied. The second pair of equations can be written in terms of $A$ :

$$
\begin{aligned}
\ddot{A} & =-\operatorname{curl} \operatorname{curl} A-J, \\
\operatorname{div} \dot{A} & =-Q .
\end{aligned}
$$

Notice that if the divergence constraint (7) is satisfied initially, then the evolution equation (6) and the conservation of charge (5) guarantee that the divergence constraint is satisfied at all times. The usual boundary condition for $A$ is that its tangential component $A_{\mathrm{T}}$ on $\partial U$ should vanish. Then $E$ also has zero tangential component on $\partial U$ - this is the perfect conductor boundary condition.

The second order evolution equation (6) can be cast into the above framework. We take $H$ to be the space of square integrable vector fields equipped with its usual scalar product. We define:

$$
X=\left\{u \in H \quad: \operatorname{curl} u \in H \text { and } u_{\mathrm{T}}=0\right\},
$$

It is a non-trivial fact that the vanishing of the tangential component $u_{\mathrm{T}}$ of $u$ on the boundary makes sense in this topology [48]. The bilinear form $a$ is defined on $X$ by:

$$
a(u, v)=\langle\operatorname{curl} u, \operatorname{curl} v\rangle .
$$

\subsection{Convergence theory for linear equations}

We now study the construction of approximate solutions to the abstract wave equation by the Galerkin method. The literature on the topic includes [46], [47], [31] and [63]. I was particularly inspired by [42].

We suppose we are given a family $\left(X_{h}\right)$ of finite dimensional subspaces of $X$ with the property that for all $v \in X$ :

$$
\lim _{h \rightarrow 0} \inf _{v_{h} \in X_{h}}\left\|v-v_{h}\right\|_{X}=0 .
$$

We now look for $u_{h} \in \mathrm{C}^{1}\left([0, T] ; X_{h}\right)$ such that $\dot{u}_{h}$ is absolutely continuous and for almost every $t \in[0, T]$ we have:

$$
\forall u^{\prime} \in X_{h} \quad\left\langle\ddot{u}_{h}(t), u^{\prime}\right\rangle=-a\left(u_{h}(t), u^{\prime}\right)+\left\langle f(t), u^{\prime}\right\rangle .
$$

Initial conditions are approximated in $X_{h}$. This problem has a unique solution by ODE theory.

The first result is a stability estimate. We denote the time-derivative of a quantity $\mathcal{U}$, by $\mathcal{U}^{\bullet}$. We have:

$$
\mathcal{E}\left(u_{h}\right)^{\bullet}(t)=\left\langle f(t), \dot{u}_{h}(t)\right\rangle \leq 2^{1 / 2}|f(t)| \mathcal{E}(u)(t)^{1 / 2} .
$$

From this it follows that:

$$
\mathcal{E}(u)(t)^{1 / 2}-\mathcal{E}(u)(0)^{1 / 2} \leq 1 / 2^{1 / 2} \int_{0}^{t}|f(s)| \mathrm{d} s .
$$

From the stability one can conclude that at a subsequence converges weakstar in the space of bounded energy functions:

$$
\left\{u \in \mathrm{L}^{\infty}([0, T] ; X): \dot{u} \in \mathrm{L}^{\infty}([0, T] ; H)\right\},
$$

where the time derivative is defined a priori in the sense of $X$-valued distributions. 
One can show that the weak limit is a solution. Proving uniqueness and continuity/differentiability of the solution requires extra work. Assuming now that these results for the continuous problem have been established we wish to examine the strong convergence of $u_{h}$ to $u$ in the natural norm provided by the energy.

Set $e_{h}=u-u_{h}$. We have for any $e^{\prime} \in X_{h}$ :

$$
\left\langle\ddot{e}_{h}(t), e^{\prime}\right\rangle+a\left(e_{h}(t), e^{\prime}\right)=0 .
$$

Let $v_{h}$ be any smooth enough function $[0, T] \rightarrow X_{h}$. At almost every time $t$ we have:

$$
\begin{aligned}
\mathcal{E}(u)^{\bullet} & =\left\langle\ddot{e}_{h}, \dot{u}-\dot{u}_{h}\right\rangle+a\left(e_{h}, \dot{u}-\dot{u}_{h}\right), \\
& =\left\langle\ddot{e}_{h}, \dot{u}-\dot{v}_{h}\right\rangle+a\left(e_{h}, \dot{u}-\dot{v}_{h}\right), \\
& =\left\langle\dot{e}_{h}, \dot{u}-\dot{v}_{h}\right\rangle^{\bullet}-\left\langle\dot{e}_{h}, \ddot{u}-\ddot{v}_{h}\right\rangle+a\left(e_{h}, \dot{u}-\dot{v}_{h}\right) .
\end{aligned}
$$

The two last terms are bounded by:

$$
\left|\dot{e}_{h}\right|\left|\ddot{u}-\ddot{v}_{h}\right|+a\left(e_{h}, e_{h}\right)^{1 / 2} a\left(\dot{u}-\dot{v}_{h}, \dot{u}-\dot{v}_{h}\right)^{1 / 2} \leq 2 \mathcal{E}\left(e_{h}\right)^{1 / 2} \mathcal{E}\left(\dot{u}-\dot{v}_{h}\right)^{1 / 2} .
$$

Inserting this in (11) and integrating gives:

$\mathcal{E}\left(e_{h}\right)(t)-\mathcal{E}\left(e_{h}\right)(0) \leq\left[\left\langle\dot{e}_{h}, \dot{u}-\dot{v}_{h}\right\rangle\right]_{0}^{t}+2\left(\int_{0}^{t} \mathcal{E}\left(e_{h}\right)\right)^{1 / 2}\left(\int_{0}^{t} \mathcal{E}\left(\dot{u}-\dot{v}_{h}\right)\right)^{1 / 2}$

The first term on the right hand side is bounded by:

$$
2 \mathcal{E}\left(e_{h}\right)(t)^{1 / 2} \mathcal{E}\left(u-v_{h}\right)(t)^{1 / 2}+2 \mathcal{E}\left(e_{h}\right)(0)^{1 / 2} \mathcal{E}\left(u-v_{h}\right)(0)^{1 / 2} .
$$

Thus we get:

$$
\begin{gathered}
(1 / 2) \mathcal{E}\left(e_{h}\right)(t) \leq 2 \mathcal{E}\left(u-v_{h}\right)(t)+2 \mathcal{E}\left(e_{h}\right)(0)+\mathcal{E}\left(u-v_{h}\right)(0)+ \\
\int_{0}^{t} \mathcal{E}\left(e_{h}\right)+\int_{0}^{t} \mathcal{E}\left(\dot{u}-\dot{v}_{h}\right) .
\end{gathered}
$$

Gronwall's lemma then shows that there is $C$ depending only on $T$ such that:

$$
\sup _{0 \leq t \leq T} \mathcal{E}\left(e_{h}\right)(t) \leq C\left(\mathcal{E}\left(e_{h}\right)(0)+\sup _{0 \leq t \leq T} \mathcal{E}\left(u-v_{h}\right)(t)+\int_{0}^{T} \mathcal{E}\left(\dot{u}-\dot{v}_{h}\right)(s) \mathrm{d} s\right) .
$$

For any $p$ denote:

$$
\mathcal{X}^{p}=\left\{u \in C^{p}([0, T] ; X): u^{(p+1)} \in C([0, T] ; H)\right\} .
$$

We also define:

$$
\mathcal{X}_{h}^{p}=\left\{u \in C^{p+1}\left([0, T] ; X_{h}\right)\right\} .
$$

One checks that for all $p$ and for all $u \in \mathcal{X}^{p}$ one has :

$$
\lim _{h \rightarrow 0} \inf _{v_{h} \in \mathcal{X}_{h}^{p}}\left\|u-v_{h}\right\|_{\mathcal{X}^{p}}=0 .
$$

To get convergence in (14) it is therefore enough to have $u$ in $\mathcal{X}^{1}$. This is a stronger condition than just to be in the energy space $\mathcal{X}^{0}$. It is guaranteed if the data is more regular than we assumed initially: $\dot{u}(0) \in$ $X, \ddot{u}(0) \in H$ and $\dot{f} \in \mathrm{L}^{1}(0, T ; H)$ will do, since then $\dot{u}$ satisfies an abstract 
wave equation, with finite energy initial data and with forcing term $\dot{f}$. Remark also that if $u(0)$ is in the domain of $a$, that is $u(0) \in X$ and :

$$
\sup _{u^{\prime} \in X}\left|a\left(u(0), u^{\prime}\right)\right| /\left|u^{\prime}\right|<+\infty,
$$

then the abstract wave equation for $u$ guarantees that the condition $\ddot{u}(0) \in$ $H$ is satisfied.

Finally since we have stability in $\mathcal{X}^{0}$ for data of the type $u_{0} \in X$, $\dot{u}_{0} \in H$ and $f \in \mathrm{L}^{1}(0, T ; H)$, and norm-convergence in $\mathcal{X}^{0}$ for a dense subset of these, we get norm-convergence in $\mathcal{X}^{0}$ in general.

Orders of convergence are usually deduced from (14) by assuming a certain amount of extra regularity on the data, deducing regularity of $u$ in various Sobolev spaces, letting $v_{h}=\Pi_{h} u$ for a certain $X_{h}$-valued interpolation operator $\Pi_{h}$ and using known error estimates for $u-\Pi_{h} u$ given this Sobolev regularity of $u$.

We now turn to time discretization of (8). We will consider only the most popular scheme which is the leap-frog scheme, also called the Störmer-Verlet scheme. The time-step is denoted $\Delta t$. Approximations $u_{h}^{n} \approx u_{h}(n \Delta t)$ are obtained by the recursion formula (for the time being we omit the subscript $h$ ):

$$
\left\langle\frac{u^{n+1}-2 u^{n}+u^{n-1}}{(\Delta t)^{2}}, u^{\prime}\right\rangle=-a\left(u^{n}, u^{\prime}\right)+\left\langle f^{n}, u^{\prime}\right\rangle .
$$

Here we put $f^{n}=f(n \Delta t)$. Inserting:

$$
u^{\prime}=u^{n+1}-u^{n-1}=\left(u^{n+1}-u^{n}\right)+\left(u^{n}-u^{n-1}\right),
$$

into this formula gives:

$$
\begin{gathered}
\left|\frac{u^{n+1}-u^{n}}{\Delta t}\right|^{2}-\left|\frac{u^{n}-u^{n-1}}{\Delta t}\right|^{2}= \\
-a\left(u^{n}, u^{n+1}\right)+a\left(u^{n}, u^{n-1}\right)+ \\
\left\langle f^{n}, u^{n+1}-u^{n-1}\right\rangle .
\end{gathered}
$$

We introduce the discrete energy of $\left(u^{n}\right)$

$$
\mathcal{E}^{n+1 / 2}=1 / 2\left|\frac{u^{n+1}-u^{n}}{\Delta t}\right|^{2}+1 / 2 a\left(u^{n}, u^{n+1}\right) .
$$

When $f$ is zero it is conserved. Unfortunately it need not even be positive due to the second term. We can write:

$$
2 a\left(u^{n}, u^{n+1}\right)=a\left(u^{n+1}, u^{n+1}\right)+a\left(u^{n}, u^{n}\right)-a\left(u^{n+1}-u^{n}, u^{n+1}-u^{n}\right) .
$$

We wish to control the third term on the right hand side. In finite element theory, if $h$ denotes the mesh-width (the largest diameter of a cell of a given mesh), and $a$ is the bilinear form associated with a second order operator (so is continuous on $\mathrm{H}^{1}(U)$ ) there exists a constant $C>0$ such that for all $h$ and all $u, v \in X_{h}$ :

$$
a(u, v) \leq C h^{-2}|u||v| .
$$

Such bounds are called inverse estimates. Notice that the bound blows up as $h \rightarrow 0$, in accordance with the possible non-continuity of $a$ on $\mathrm{L}^{2}(U)$. We henceforth suppose that such an estimate exists in our abstract setting. Suppose also that with this constant $C, \Delta t$ is chosen so small that:

$$
1-C h^{-2}(\Delta t)^{2} / 2 \geq \delta>0 .
$$


In finite element theory the interpretation is that $\Delta t$ must be smaller than something comparable to the mesh-width $h$. It is known as the CFL condition. Then we have:

$$
\mathcal{E}^{n+1 / 2} \geq \delta / 2\left|\frac{u^{n+1}-u^{n}}{\Delta t}\right|^{2}+1 / 4\left(a\left(u^{n+1}, u^{n+1}\right)+a\left(u^{n}, u^{n}\right)\right),
$$

consisting of non-negative terms.

From (16) we deduce:

$$
\begin{aligned}
2\left(\mathcal{E}^{n+1 / 2}-\mathcal{E}^{n-1 / 2}\right) & =\left\langle f^{n}, u^{n+1}-u^{n}\right\rangle+\left\langle f^{n}, u^{n}-u^{n-1}\right\rangle, \\
& \leq(2 / \delta)^{1 / 2} \Delta t\left|f^{n}\right|\left(\left(\mathcal{E}^{n+1 / 2}\right)^{1 / 2}+\left(\mathcal{E}^{n-1 / 2}\right)^{1 / 2}\right) .
\end{aligned}
$$

Therefore:

$$
\left(\mathcal{E}^{n+1 / 2}\right)^{1 / 2}-\left(\mathcal{E}^{n-1 / 2}\right)^{1 / 2} \leq 1 /(2 \delta)^{1 / 2}\left|f^{n}\right| \Delta t,
$$

yielding:

$$
\left(\mathcal{E}^{n+1 / 2}\right)^{1 / 2}-\left(\mathcal{E}^{1 / 2}\right)^{1 / 2} \leq 1 /(2 \delta)^{1 / 2} \sum_{k=1}^{n}\left|f^{k}\right| \Delta t .
$$

This is a stability estimate comparable to (9) but we must require that $f$ is Riemann integrable (with values in $H$ ) rather than merely Lebesgue integrable.

Next we wish to establish convergence. We estimate the distance from the semidiscrete $u_{h}(n \Delta t)$ to the fully discrete $u_{h}^{n}$. Set $e_{h}^{n}=u_{h}(n \Delta t)-u_{h}^{n}$. It turns out that $e_{h}^{n}$ satisfies:

$$
\left\langle\frac{e_{h}^{n+1}-2 e_{h}^{n}+e_{h}^{n-1}}{(\Delta t)^{2}}, e^{\prime}\right\rangle=-a\left(e_{h}^{n}, u^{\prime}\right)+\left\langle\epsilon_{h}^{n}, e^{\prime}\right\rangle,
$$

with:

$$
\epsilon_{h}^{n}=\frac{u_{h}((n+1) \Delta t)-2 u_{h}(n \Delta t)+u_{h}((n-1) \Delta t)}{(\Delta t)^{2}}-\ddot{u}_{h}(n \Delta t) .
$$

Put:

$$
\hat{\mathcal{E}}_{h}^{n+1 / 2}=1 / 2\left|\frac{e_{h}^{n+1}-e_{h}^{n}}{\Delta t}\right|^{2}+1 / 2 a\left(e_{h}^{n}, e_{h}^{n+1}\right) .
$$

As before we get:

$$
\left(\hat{\mathcal{E}}^{n+1 / 2}\right)^{1 / 2}-\left(\hat{\mathcal{E}}^{1 / 2}\right)^{1 / 2} \leq 1 /(2 \delta)^{1 / 2} \sum_{k=1}^{n}\left|\epsilon_{h}^{k}\right| \Delta t .
$$

We want to bound $\epsilon_{h}^{k}$. One has the formula:

$$
\epsilon_{h}^{k}=\int_{0}^{1}\left(\ddot{u}_{h}((k+s) \Delta t)-2 \ddot{u}_{h}(k \Delta t)+\ddot{u}_{h}((k-s) \Delta t)\right)(1-s) \mathrm{d} s .
$$

For any $T>0$ if $\dot{u}(0) \in X, \ddot{u}(0) \in H$ and $\dot{f} \in \mathrm{L}^{1}([0, T] ; H)$ we get convergence of $u_{h}$ to $u$ in $\mathrm{C}^{2}([0, T] ; H)$. Actually we need to be careful with the way initial conditions are handled to guarantee this. We need to choose $u_{h}(0) \in X_{h}$ and $\dot{u}_{h}(0) \in X_{h}$ so that:

$$
\begin{aligned}
& u_{h}(0) \rightarrow u(0) \text { in } X, \\
& \dot{u}_{h}(0) \rightarrow \dot{u}(0) \text { in } H,
\end{aligned}
$$


but in addition we need that whenever $\dot{u}(0) \in X$ and $\ddot{u}(0) \in H$ we have:

$$
\begin{aligned}
& \dot{u}_{h}(0) \rightarrow \dot{u}(0) \text { in } X, \\
& \ddot{u}_{h}(0) \rightarrow \ddot{u}(0) \text { in } H .
\end{aligned}
$$

To guarantee $(20)$ and (21) we can approximate $\dot{u}_{h}(0)$ using projections $H \rightarrow X_{h}$ which are stable not only in $H$ but also in $X$. We will comment later on the construction of such projections for finite element spaces. To guarantee (19) and (22) we can use the scalar product $\langle\cdot, \cdot\rangle+a(\cdot, \cdot)$ to project $u(0)$ to $X_{h}$. Then we have for all $u^{\prime} \in X_{h}$ :

$$
\begin{aligned}
\left\langle\ddot{u}_{h}(0), u^{\prime}\right\rangle & =-a\left(u_{h}(0), u^{\prime}\right)+\left\langle f(0), u^{\prime}\right\rangle, \\
& =-a\left(u(0), u^{\prime}\right)-\left\langle u(0), u^{\prime}\right\rangle+\left\langle u_{h}(0), u^{\prime}\right\rangle+\left\langle f(0), u^{\prime}\right\rangle, \\
& =\left\langle\ddot{u}(0), u^{\prime}\right\rangle-\left\langle u(0)-u_{h}(0), u^{\prime}\right\rangle,
\end{aligned}
$$

from which (22) follows.

Since now $u_{h}$ converges to $u$ in $\mathrm{C}^{2}([0, T] ; H)$ and $\ddot{u}:[0, T] \rightarrow H$ is uniformly continuous, we have:

$$
\sup \left\{\left|\ddot{u}_{h}\left(t^{\prime}\right)-\ddot{u}_{h}(t)\right|: 0 \leq t, t^{\prime} \leq T \text { and }\left|t^{\prime}-t\right| \leq \Delta t\right\} \rightarrow 0,
$$

which thanks to (18) gives:

$$
\sum_{k=1}^{\lfloor T / \Delta t\rfloor}\left|\epsilon_{h}^{k}\right| \Delta t \rightarrow 0 .
$$

From this one deduces convergence of the linear interpolant of the sequence $\left(u_{h}^{n}\right)$ to $u$ in the space of bounded energy functions defined in (10) under the CFL condition.

Finally stability for all finite energy initial conditions and Riemann integrable $f$ together with convergence for a dense subset of such data imply convergence for all such data.

\subsection{Consistency}

Usually the bilinear forms involved are not just restricted to the Galerkin space, they are also approximated. This is necessary when material parameters vary for instance. It can also have advantages. Equation (15) requires solving a linear system involving the mass matrix (the matrix of $\langle\cdot, \cdot\rangle$ in the chosen basis) at each time-step. If the mass matrix can be approximated by a diagonal matrix, without loss of accuracy, a lot of work is saved. We investigate such approximations in general. We start with stationary problems.

Let $X, Y$ be Banach spaces and $a$ a continuous bilinear form $X \times Y \rightarrow$ $\mathbb{R}$. It induces a map:

$$
A:\left\{\begin{aligned}
X & \rightarrow Y^{\star} \\
u & \mapsto a(u, \cdot) .
\end{aligned}\right.
$$

If it is invertible the following number is positive:

$$
\left\|A^{-1}\right\|^{-1}=\inf _{u \in X} \sup _{v \in Y} \frac{|a(u, v)|}{\|u\|\|v\|} .
$$

Suppose $\left(X_{h}\right)$ and $\left(Y_{h}\right)$ are families of finite dimensional subspaces of $X$ and $Y$ respectively. We suppose $\operatorname{dim} X_{h}=\operatorname{dim} Y_{h}$. We suppose that $\left(X_{h}\right)$ is approximating in the sense that:

$$
\forall u \in X \quad \inf _{u_{h} \in X_{h}}\left\|u-u_{h}\right\| \rightarrow 0,
$$


We make a similar hypothesis for $\left(Y_{h}\right)$. Let $a_{h}$ be bilinear forms $X_{h} \times Y_{h} \rightarrow$ $\mathbb{R}$.

Given $l \in Y^{\star}$ we want to find approximations to the solution $u \in X$ to:

$$
\forall v \in Y \quad a(u, v)=l(v) .
$$

We construct linear forms $l_{h} \in Y_{h}^{\star}$ which approximate the restriction of $l$. We find $u_{h} \in X_{h}$ such that:

$$
\forall v \in Y_{h} \quad a_{h}\left(u_{h}, v\right)=l_{h}(v) .
$$

Two conditions play a crucial role in the analysis of the convergence of $u_{h}$ : The inf-sup condition [7][14], and consistency [58].

- Inf-sup condition. There is $C>0$ such that for all $h$ :

$$
\inf _{u \in X_{h}} \sup _{v \in Y_{h}} \frac{\left|a_{h}(u, v)\right|}{\|u\|\|v\|} \geq 1 / C .
$$

- Consistency. For any $u \in X$ there is a sequence $\tilde{u}_{h} \in X_{h}$ such that $\tilde{u}_{h} \rightarrow u$ in $X$ as $h \rightarrow 0$ and:

$$
\sup _{v \in Y_{h}} \frac{\left|a(u, v)-a_{h}\left(\tilde{u}_{h}, v\right)\right|}{\|v\|} \rightarrow 0 \text { as } h \rightarrow 0 .
$$

Going back to the analysis of (23), we suppose that the inf-sup condition and the consistency hold. Choosing $\tilde{u}_{h} \in X_{h}$ as in the definition of consistency we have:

$$
\begin{aligned}
\left\|u_{h}-\tilde{u}_{h}\right\| & \leq C \sup _{v \in Y_{h}} \frac{\left|a_{h}\left(u_{h}-\tilde{u}_{h}, v\right)\right|}{\|v\|}, \\
& \leq C \sup _{v \in Y_{h}} \frac{\left|l_{h}(v)-l(v)+a(u, v)-a_{h}\left(\tilde{u}_{h}, v\right)\right|}{\|v\|}, \\
& \leq C\left(\left\|l-l_{h}\right\|_{Y_{h}^{\star}}+\sup _{v \in Y_{h}} \frac{\left|a(u, v)-a_{h}\left(\tilde{u}_{h}, v\right)\right|}{\|v\|}\right) .
\end{aligned}
$$

We suppose $\left\|l-l_{h}\right\|_{Y_{h}^{\star}} \rightarrow 0$ and obtain $u_{h} \rightarrow u$.

A diagonal argument shows that it is enough to ensure the consistency requirement for all $u$ in a dense subset $X_{0}$ of $X$. There usually are such dense subsets for which one obtains rather explicit rates of convergence in (24). When the solution to the continuous problem is in such a subset, the above computation gives convergence rates for $u_{h} \rightarrow u$.

Reciprocally if convergence $u_{h} \rightarrow u$ is to hold for any $l \in Y^{\star}$ (with simply $l_{h}=\left.l\right|_{Y_{h}}$ ) then the inf-sup condition and the consistency must hold. We prove this. First concerning the inf-sup condition we do the following. We define the mappings:

$$
A_{h}:\left\{\begin{aligned}
X_{h} & \rightarrow Y_{h}^{\star} \\
u & \mapsto a_{h}(u, \cdot)
\end{aligned}\right.
$$

They must be invertible. Let $r_{h}: Y^{\star} \rightarrow Y_{h}^{\star}$ be the restriction map. We suppose that for all $l \in Y^{\star}$ we have:

$$
A_{h}^{-1} r_{h} l \rightarrow A^{-1} l \text {. }
$$

By the uniform boundedness principle there must be $C$ such that for all $h$ :

$$
\left\|A_{h}^{-1} r_{h}\right\|_{Y^{\star} \rightarrow X} \leq C .
$$


By the Hahn-Banach theorem it follows that:

$$
\left\|A_{h}^{-1}\right\|_{Y_{h}^{\star} \rightarrow X_{h}} \leq C .
$$

This can be rephrased as:

$$
\inf _{u \in X_{h}} \sup _{v \in Y_{h}} \frac{\left|a_{h}(u, v)\right|}{\|u\|\|v\|} \geq 1 / C .
$$

So we get the inf-sup condition. That consistency must hold is clear: For a given $u$ simply let $\tilde{u}_{h}=u_{h}$ be the discrete solution:

$$
\forall v \in Y_{h} \quad a_{h}\left(u_{h}, v\right)=a(u, v) .
$$

Since the inf-sup condition is a stability condition we get an instance of the Lax equivalence principle: convergence is equivalent to stability and consistency.

Usually there is $C>0$ such that for all $h$ :

$$
\forall u \in X_{h} \forall v \in Y_{h} \quad\left|a_{h}(u, v)\right| \leq C\|u\|\|v\| .
$$

If the method is consistent and this equiboundedness condition holds, we have that for any $u \in X$ and any sequence $\tilde{u}_{h} \in X_{h}$ such that $\tilde{u}_{h} \rightarrow u$ in $X$ as $h \rightarrow 0$ :

$$
\sup _{v \in Y_{h}} \frac{\left|a(u, v)-a_{h}\left(\tilde{u}_{h}, v\right)\right|}{\|v\|} \rightarrow 0 \text { as } h \rightarrow 0 .
$$

Reciprocally if this last condition holds then (25) holds and the method is consistent. Indeed suppose (25) does not hold. Then there are subsequences $u_{h} \in X_{h}, v_{h} \in X_{h}$ such that $\left|a_{h}\left(u_{h}, v_{h}\right)\right|=1,\left\|u_{h}\right\| \rightarrow 0$ and $\left\|v_{h}\right\|=1$. This contradicts (26).

We now return to the wave equation, in its abstract formulation. We suppose we have constructed bilinear forms:

$$
\langle\cdot, \cdot\rangle_{h}, a_{h}(\cdot, \cdot): X_{h} \times X_{h} \rightarrow \mathbb{R},
$$

with the properties that for some $C$ we have for all $h$ :

$$
\forall u \in X_{h} \quad 1 / C\langle u, u\rangle \leq\langle u, u\rangle_{h} \leq C\langle u, u\rangle,
$$

and:

$$
\forall u \in X_{h} \quad 1 / C a(u, u) \leq a_{h}(u, u) \leq C a(u, u) .
$$

We require $\langle\cdot, \cdot\rangle_{h}$ to be a consistent discretization of $\langle\cdot, \cdot\rangle$ with respect to the $H$ norm and $a_{h}(\cdot, \cdot)$ to be a consistent discretization of $a(\cdot, \cdot)$ with respect to the $X$ norm.

The new semidiscrete equation we solve is:

$$
\left\langle\ddot{u}_{h}(t), u^{\prime}\right\rangle_{h}=-a_{h}\left(u_{h}(t), u^{\prime}\right)+\left\langle f(t), u^{\prime}\right\rangle .
$$

Notice that we don't do anything with the $f$ term, though I suppose this is possible.

Let $\mathcal{E}_{h}$ be the corresponding energy:

$$
\mathcal{E}_{h}(u)(t)=1 / 2\langle\dot{u}(t), \dot{u}(t)\rangle_{h}+1 / 2 a_{h}(u(t), u(t)) .
$$

We get stability for this energy as before. Stability for the true energy follows from the comparison estimates (27) and (28). 
To get convergence we first make the usual extra assumptions that $u(0) \in X$ is in the domain of $a, \dot{u}(0) \in X$ and $\dot{f} \in \mathrm{L}^{1}([0, T] ; H)$. They guarantee that $u \in \mathcal{X}^{1}$. We compare $u_{h}$ with some function $v_{h}:[0, T] \rightarrow$ $X_{h}$. We have:

$$
\begin{aligned}
\mathcal{E}_{h}\left(u_{h}-v_{h}\right)^{\bullet}= & \left\langle\ddot{u}_{h}-\ddot{v}_{h}, \dot{u}_{h}-\dot{v}_{h}\right\rangle_{h}+a_{h}\left(u_{h}-v_{h}, \dot{u}_{h}-\dot{v}_{h}\right), \\
= & \left\langle\ddot{u}, \dot{u}_{h}-\dot{v}_{h}\right\rangle-\left\langle\ddot{v}_{h}, \dot{u}_{h}-\dot{v}_{h}\right\rangle_{h}+ \\
& a\left(u, \dot{u}_{h}-\dot{v}_{h}\right)-a_{h}\left(v_{h}, \dot{u}_{h}-\dot{v}_{h}\right) .
\end{aligned}
$$

We can ensure the convergence of $v_{h}$ to $u$ in $\mathcal{X}^{1}$. We also have boundedness of $\left(u_{h}\right)$ in the space $\mathcal{X}^{1}$ by the stability argument applied to the time-differentiated equation. Consistency augmented by a compactness argument then shows:

$$
\sup _{0 \leq t \leq T} \mathcal{E}_{h}\left(u_{h}-v_{h}\right)^{\bullet}(t) \rightarrow 0 .
$$

This gives convergence in $\mathcal{X}^{0}$. As usual stability for general data and convergence on a dense subset give convergence in general.

The treatment of the time-discretized case can be carried out along the path sketched for the case of exact bilinear forms.

Finally we include a proof that the procedure known as mass lumping gives, on scalar degree one continuous elements, a stable and consistent discretization of the $\mathrm{L}^{2}$ scalar product on functions. The techniques involved are detailed for instance in [30]. For the extension to higher order finite element methods see [32], for the case of vectorial finite elements in relation to the Yee scheme see [47].

The computational domain $U \subseteq \mathbb{R}^{n}$ is equipped with a regular simplicial mesh $\mathcal{T}_{h}$. The space $X_{h}$ consists of the functions that are continuous and piecewise affine with respect to $\mathcal{T}_{h}$. The parameter $h$ is identified with the largest diameter of a cell of $\mathcal{T}_{h}$. We denote by $I_{h}$ the nodal interpolator, which is defined on continuous functions and is a projection onto $X_{h}$. The mass-lumped $\mathrm{L}^{2}$ product is defined on continuous functions by:

$$
\langle u, v\rangle_{h}=\int I_{h}(u v)
$$

Notice that in the standard basis of $X_{h}$, the matrix of this bilinear form is diagonal. A rather explicit formula is:

$$
\langle u, v\rangle_{h}=\sum_{T \in \mathcal{T}_{h}} \frac{|T|}{n+1} \sum_{x \in T} u(x) v(x) .
$$

Here the points $x$ in the sum are the $n+1$ vertices of $T$.

Consider a reference element $\hat{T}$. By equivalence of norms in finite dimensions and unisolvence of the vertex degrees of freedom on affine functions, there are constants $C, C^{\prime}$ such that for any affine function $u$ on $T$ :

$$
1 / C^{\prime} \int_{\hat{T}}|u|^{2} \leq \frac{|\hat{T}|}{n+1} \sum_{x \in \hat{T}}|u(x)|^{2} \leq C \int_{\hat{T}}|u|^{2} .
$$

Transporting this to all elements $T$ of $\mathcal{T}_{h}$ and adding gives, for all $h$ and all $u \in X_{h}$ :

$$
1 / C^{\prime}\langle u, u\rangle \leq\langle u, u\rangle_{h} \leq C\langle u, u\rangle
$$

which ensures stability and equiboundedness.

Let $l>n / 2$, which ensures that $\mathrm{H}^{l}(U)$ is continuously injected into the space of continuous functions on $\bar{U}$. Standard Sobolev norms will be 
denoted $\|\cdot\|$ whereas seminorms are denoted $|\cdot|$. We shall prove that there is $C>0$ such that for all $u \in \mathrm{H}^{l}(U)$, all $h$ and all $v \in X_{h}$ :

$$
\left|\langle u, v\rangle-\langle u, v\rangle_{h}\right| \leq C h\|u\|_{\mathrm{H}^{l}(U)}\|v\|_{\mathrm{L}^{2}(U)} .
$$

We want to estimate the errors $E_{T}(u v)$ with $E_{T}$ defined by:

$$
E_{T}(w)=\int_{T} w-\frac{|T|}{n+1} \sum_{x \in T} w(x) .
$$

The above integration rule is exact for polynomials of degree at most 1. Working on the reference element, a Bramble Hilbert lemma gives an estimate of the form, for all $w \in \mathrm{H}^{l}(\hat{T})$ :

$$
\left|E_{\hat{T}}(w)\right| \leq C\left(|w|_{\mathrm{H}^{2}(\hat{T})}^{2}+|w|_{\mathrm{H}^{l}(\hat{T})}^{2}\right)^{1 / 2}
$$

We apply a Leibniz rule for differentiation of products. For $u \in \mathrm{H}^{l}(\hat{T})$ and $v$ confined to the finite-dimensional space of affine functions on $\hat{T}$, we may deduce:

$$
\left|E_{\hat{T}}(u v)\right| \leq C\left(|u|_{\mathrm{H}^{1}(\hat{T})}^{2}+\cdots+|u|_{\mathrm{H}^{l}(\hat{T})}^{2}\right)^{1 / 2}\|v\|_{\mathrm{L}^{2}(\hat{T})} .
$$

Transporting this estimate to a simplex $T$ of diameter $h$ gives:

$$
\left|E_{T}(u v)\right| \leq C h\|u\|_{\mathrm{H}^{l}(T)}\|v\|_{\mathrm{L}^{2}(T)} .
$$

Summing these estimates and applying a Cauchy-Schwartz inequality gives (30).

The same arguments also show:

$$
\left|\langle u, v\rangle-\langle u, v\rangle_{h}\right| \leq C h^{2}\|u\|_{\mathrm{H}^{l}(U)}\|v\|_{\mathrm{H}^{1}(U)} .
$$

\subsection{Eigenvalue approximation}

Linear evolution problems are closely linked to eigenvalue problems. For instance the solution of the wave equation (1) can be expressed quite simply in terms of the eigenvalues and eigenvectors of the Laplace operator. Similarly the discrete solutions of the wave equation obtained by the Galerkin method can be expressed in terms of discrete eigenvalues and eigenvectors. In this section we comment on the relation between continuous and discrete eigenproblems. The emphasis is on problems of Maxwell type, for which the bilinear form $a$ will have a large kernel. References on the subject include [43], [9], [10] and [19]. For $p$-version finite elements there are some new developments [33] [41].

We introduce the following notations, which construct a decomposition of $X$ generalizing the Helmholtz decomposition of vectorfields (into a gradient and a curl) to our abstract setting. We let $W$ be the kernel of $a$ defined by:

$$
W=\left\{u \in X \quad: \forall u^{\prime} \in X \quad a\left(u, u^{\prime}\right)=0\right\} .
$$

Notice that by (3) we have, for all $u \in X$ :

$$
u \in W \Longleftrightarrow a(u, u)=0 .
$$

We also let $V$ be the orthogonal of $W$ in $X$ with respect to $\langle\cdot, \cdot\rangle$ :

$$
V=\{u \in X \quad: \forall w \in W \quad\langle u, w\rangle=0\} .
$$


Thus $V$ and $W$ are closed subspaces of $X$ and:

$$
X=V \oplus W .
$$

We make the additional assumption that the injection $V \rightarrow H$ is compact (when $V$ is equipped with the norm inherited from $X$ ). In particular we get a Poincaré-Friedrichs inequality. There is $C>0$ such that:

$$
\forall v \in V \quad a(v, v) \geq 1 / C|v|^{2} .
$$

Coercivity of $a$ on $V$ follows.

Notice that $W$ is a closed subspace of $H$. Let $\bar{V}$ be the orthogonal of $W$ in $H$ with respect to $\langle\cdot, \cdot\rangle$ :

$$
\bar{V}=\{u \in H \quad: \forall w \in W \quad\langle u, w\rangle=0\} .
$$

The notation is justified by the fact that $\bar{V}$ is the closure of $V$ in $H$. We have the decomposition:

$$
H=\bar{V} \oplus W .
$$

In $H$ let $P$ denote the projector with range $\bar{V}$ and kernel $W$. It is orthogonal. Notice that if $u \in X$ then $P u \in X$ since $u-P u \in W \subseteq X$. On $X$, $P$ is the projector with range $V$ and kernel $W$. It is characterized by the property that for any $u \in X, P u$ is the element of $V$ solving:

$$
\forall u^{\prime} \in V \quad a\left(P u, u^{\prime}\right)=a\left(u, u^{\prime}\right) .
$$

This equation is well posed by (31). When it holds it actually holds for all $u^{\prime} \in X$. Thus we get:

$$
\forall u, u^{\prime} \in X \quad a\left(P u, P u^{\prime}\right)=a\left(u, u^{\prime}\right) .
$$

In this setting look for pairs $(\lambda, u) \in \mathbb{R} \times X$ with $u \neq 0$ such that:

$$
\forall u^{\prime} \in X \quad a\left(u, u^{\prime}\right)=\lambda\left\langle u, u^{\prime}\right\rangle .
$$

For intance $(0, w)$ is an eigenpair of $a$ for any non-zero $w \in W ; 0$ is an eigenvalue with associated eigenspace $W$ (when $W \neq 0$ ).

We introduce the operator $T: H \rightarrow V$ defined defined as follows. For any $u \in H, T u$ is the element of $V$ satisfying:

$$
\forall u^{\prime} \in V \quad a\left(T u, u^{\prime}\right)=\left\langle u, u^{\prime}\right\rangle .
$$

That $T$ is well defined and continuous follows from the coercivity on $V$ of $a$, compare with (31). If we consider $T$ to be an operator $H \rightarrow H$ it is immediate that $T$ is compact and symmetric. Thus $H$ has an orthonormal basis consisting of eigenvectors in $X$. The eigenspace of 0 is $W$ (when $W \neq 0$ ). The non-zero eigenvalues of $T$ correspond to elements of $V$ and can be ordered in a decreasing sequence converging to 0 . Moreover $\lambda$ is a non-zero eigenvalue of $a$ iff $1 / \lambda$ is a non-zero eigenvalue of $T$ and the corresponding eigenspaces are the same.

Discrete eigenvalues are defined as follows. For each $h$ we look for pairs $(\lambda, u) \in \mathbb{R} \times X_{h}$ with $u \neq 0$ such that:

$$
\forall u^{\prime} \in X_{h} \quad a\left(u, u^{\prime}\right)=\lambda\left\langle u, u^{\prime}\right\rangle
$$

In general this eigenvalue problem is not so well-behaved: there are cases where discrete eigenvalues cluster as $h \rightarrow 0$ at values which are not eigenvalues of the continuous problem (this cannot happen if $W$ is finite dimensional). The approximation of eigenvalues requires more properties of the Galerkin spaces than does the approximation of the evolution problem. We make the following assumption: 
- There are projectors $\Pi_{h}: H \rightarrow H$ with range $X_{h}$ which are uniformly bounded in $H$ and have the property that $W$ is mapped to $W$.

We define:

$$
W_{h}=\left\{u \in X_{h}: \forall u^{\prime} \in X_{h} \quad a\left(u, u^{\prime}\right)=0\right\}
$$

and:

$$
V_{h}=\left\{u \in X_{h}: \forall w \in W_{h} \quad\langle u, w\rangle=0\right\} .
$$

Thus we have decompositions:

$$
X_{h}=V_{h} \oplus W_{h} .
$$

We have that $W_{h}=X_{h} \cap W$, but a crucial point is that $V_{h}$ need not be a subspace of $V$. Using our assumption we shall prove that $V_{h}$ is close to $V$, in a precise sense.

We have by the approximation property of $\left(X_{h}\right)$ - which holds in $X$ and therefore in $H$ by density - and the uniform boundedness of the projectors $\Pi_{h}$ :

$$
\forall u \in H \quad \Pi_{h} u \rightarrow u \text { in } H .
$$

Since the injection $V \rightarrow H$ is compact, there is a sequence $\epsilon_{h}$ converging to 0 as $h \rightarrow 0$ such that:

$$
\forall u \in V \forall h \quad\left|u-\Pi_{h} u\right| \leq \epsilon_{h}\|u\| .
$$

Choose $v_{h}$ in $V_{h}$. We aim to prove that $v_{h}-P v_{h}$ is small. Write:

$$
\left|v_{h}-P v_{h}\right| \leq\left|v_{h}-\Pi_{h} P v_{h}\right|+\left|P v_{h}-\Pi_{h} P v_{h}\right| .
$$

We have:

$$
\left|P v_{h}-\Pi_{h} P v_{h}\right| \leq \epsilon_{h}\left\|P v_{h}\right\| \leq \epsilon_{h}\left\|v_{h}\right\| .
$$

Remark that $v_{h}-P v_{h} \in W$ so, by our hypothesis on $\Pi_{h}$, we have:

$$
v_{h}-\Pi_{h} P v_{h}=\Pi_{h}\left(v_{h}-P v_{h}\right) \in W_{h} .
$$

Therefore $v_{h}$ and $P v_{h}$ are both orthogonal to $v_{h}-\Pi_{h} P v_{h}$ :

$$
\begin{aligned}
\left|v_{h}-\Pi_{h} P v_{h}\right|^{2} & =\left\langle v_{h}-\Pi_{h} P v_{h}, P v_{h}-\Pi_{h} P v_{h}\right\rangle, \\
& \leq\left|v_{h}-\Pi_{h} P v_{h}\right|\left|P v_{h}-\Pi_{h} P v_{h}\right|,
\end{aligned}
$$

so that:

$$
\left|v_{h}-\Pi_{h} P v_{h}\right| \leq\left|P v_{h}-\Pi_{h} P v_{h}\right| .
$$

Thus we get:

$$
\left|v_{h}-P v_{h}\right| \leq 2\left|P v_{h}-\Pi_{h} P v_{h}\right| \leq 2 \epsilon_{h}\left\|v_{h}\right\|
$$

In particular:

$$
\left\|v_{h}-P v_{h}\right\| \leq 2 \epsilon_{h}\left\|v_{h}\right\| .
$$
$X$ :

This property can be rephrased in terms of gaps between subspaces of

$$
\delta\left(V_{h}, V\right) \leq 2 \epsilon_{h}
$$

Since $a$ is coercive on $V$ it follows that there is $C$ such that for all $h$ :

$$
\forall v_{h} \in V_{h} \quad a\left(v_{h}, v_{h}\right) \geq 1 / C\left\|v_{h}\right\|^{2},
$$


More precisely we can write, for all small enough $h$ and all $v_{h} \in V_{h}$ :

$$
\begin{aligned}
a\left(v_{h}, v_{h}\right) & =a\left(P v_{h}, P v_{h}\right), \\
& \geq 1 / C\left\|P v_{h}\right\|^{2}, \\
& \geq 1 / C\left(1-2 \epsilon_{h}\right)^{2}\left\|v_{h}\right\|^{2} .
\end{aligned}
$$

Estimate (32) follows for small $h$. For a finite number of large $h$ one uses non-negativity of $a$.

We introduce the discrete analogue of $T$, which is the map $T_{h}: H \rightarrow V_{h}$ taking any $u \in H$ to the element $T_{h} u \in V_{h}$ such that:

$$
\forall u^{\prime} \in V_{h} \quad a\left(T_{h} u, u^{\prime}\right)=\left\langle u, u^{\prime}\right\rangle .
$$

The operator $T_{h}$ has finite-dimensional range and is symmetric. Remark that $\lambda$ is a non-zero discrete eigenvalue (of $a$ on $X_{h}$ ) iff $1 / \lambda$ is a non-zero eigenvalue of $T_{h}$. The corresponding eigenspaces are the same. In order to relate the discrete eigenvalues of $a$ to the continuous ones, we aim to prove:

$$
\left\|T-T_{h}\right\|_{H \rightarrow H} \rightarrow 0 .
$$

Let $P_{h}: X \rightarrow V_{h}$ be the projector defined by, for all $u \in X, P_{h} u$ is the element of $V_{h}$ such that:

$$
\forall u^{\prime} \in V_{h} \quad a\left(P_{h} u, u^{\prime}\right)=a\left(u, u^{\prime}\right) .
$$

This equation then holds for all $u^{\prime} \in X_{h}$. Notice that we have, for all $u \in H$ all $h$ and all $u^{\prime} \in V_{h}$ :

$$
a\left(T_{h} u, u^{\prime}\right)=\left\langle u, u^{\prime}\right\rangle=a\left(T u, u^{\prime}\right)=a\left(P_{h} T u, u^{\prime}\right) .
$$

Therefore:

$$
T_{h}=P_{h} T
$$

Now we remark that $T: H \rightarrow V$ is compact. Indeed if $\left(u_{n}\right)$ converges weakly to 0 in $H$ then we have already claimed that $\left(T u_{n}\right)$ converges strongly to 0 in $H$ (compactness of $T: H \rightarrow H$ ). In addition:

$$
a\left(T u_{n}, T u_{n}\right)=\left\langle u_{n}, T u_{n}\right\rangle \rightarrow 0 .
$$

Therefore $\left(T u_{n}\right)$ converges strongly to 0 in $X$. This shows that $T: H \rightarrow V$ is compact.

Choose $u \in V$. We shall prove that $P_{h} u \rightarrow u$ in $X$. Let $u_{h}$ be elements of $X_{h}$ converging to $u$. The following is a Cea type argument.

$$
\begin{aligned}
\left\|u-P P_{h} u\right\|^{2} & \leq C a\left(u-P P_{h} u, u-P P_{h} u\right), \\
& \leq C a\left(u-P_{h} u, u-P_{h} u\right), \\
& \leq C a\left(u-P_{h} u, u-u_{h}\right), \\
& \leq C a\left(u-P P_{h} u, u-u_{h}\right), \\
& \leq C\left\|u-P P_{h} u\right\|\left\|u-u_{h}\right\| .
\end{aligned}
$$

Hence:

$$
\left\|u-P P_{h} u\right\| \leq C\left\|u-u_{h}\right\| \rightarrow 0 .
$$

We also have stability of $P_{h}$ by (32). Hence:

$$
\left\|P_{h} u-P P_{h} u\right\| \leq C \epsilon_{h}\|u\| \rightarrow 0 .
$$


Combining the above estimates gives:

$$
\left\|u-P_{h} u\right\| \rightarrow 0
$$

Compactness of $T: H \rightarrow V$ and pointwise convergence of $P_{h}: V \rightarrow X$ combine to prove that:

$$
\left\|T-T_{h}\right\|_{H \rightarrow X} \rightarrow 0
$$

which is slightly stronger than we need. Whenever (33) holds we have (see [8]) that for any $\delta>0$ not is the spectrum of $T$, the eigenvalues of $T_{h}$ which are above $\delta$ can be split into sets, each set converging to an eigenvalue of $T$ (in the sense of Hausdorff distance). If one such set converges to say $\lambda$ the sum of the corresponding discrete eigenspaces converges to the continuous eigenspace of $\lambda$ (in the sense of gaps between subspaces of $H$ ).

\section{Construction of finite element spaces}

We expressed Maxwell's equations in terms of vectorfields. The relevant operators on scalar and vectorfields are the gradient, the curl and the divergence. For any of these three operators op one defines:

$$
\mathrm{H}_{\mathrm{op}}=\left\{u \in \mathrm{L}^{2} \quad: \text { op } u \in \mathrm{L}^{2}\right\} \text {. }
$$

We then have a diagram of spaces linked by operators:

$$
\mathrm{H}_{\text {grad }} \stackrel{\text { grad }}{\longrightarrow} \mathrm{H}_{\text {curl }} \stackrel{\text { curl }}{\longrightarrow} \mathrm{H}_{\text {div }} \stackrel{\text { div }}{\longrightarrow} \mathrm{H}
$$

An important property is that the compositions of any two consecutive operators is 0 . One says that the spaces form a complex.

To obtain good discretizations of Maxwell's equations one now tends not only to construct subspaces $X_{h}^{1}$ of $\mathrm{H}_{\text {curl }}$ but also subspaces $X_{h}^{0}$ of $\mathrm{H}_{\text {grad }}, X_{h}^{2}$ of $\mathrm{H}_{\mathrm{div}}$ and $X_{h}^{3}$ of $\mathrm{H}$, also forming, for each $h$ a complex for the same operators. Moreover one wishes to relate the two complexes by projections onto these subspaces, forming a commuting diagram:

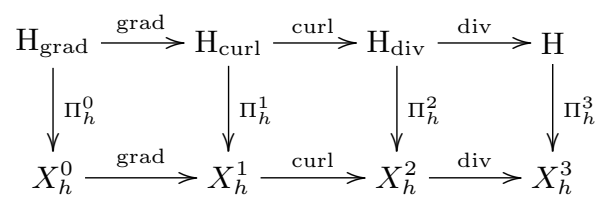

Commutativity of the diagram means that one can follow the arrows along any path between two spaces and still obtain the same operator between these spaces.

We first give some results concerning such diagrams of complexes and tensor product constructions. Then we recall the definition and basic properties of differential forms on manifolds. They provide a more flexible framework than vector fields. We also define cochains associated with decompositions of space into cells which are at the basis of discretizations. Finally we apply these concepts to the construction of spaces $X_{h}^{k}$ as above. 


\section{$2.1 \quad$ Algebra}

Homological algebra We now recall some definitions and facts from homological algebra, and refer the reader to Lang [45] and Gelfand-Manin [36] for thorough expositions. In this paper by a complex we mean a sequence $A^{\bullet}$ of vectorspaces equipped with linear operators $d^{k}: A^{k} \rightarrow$ $A^{k+1}$ called differentials and satisfying, for each $k, d^{k+1} d^{k}=0$. The cohomology group $\mathrm{H}^{k} A^{\bullet}$ is (the vectorspace) defined by:

$$
\mathrm{H}^{k} A^{\bullet}=\left(\operatorname{ker} d^{k}: A^{k} \rightarrow A^{k+1}\right) /\left(\operatorname{im} d^{k-1}: A^{k-1} \rightarrow A^{k}\right)
$$

Most often the index $k$ in $d^{k}$ is dropped and the complex is represented by a diagram:

$$
\cdots \rightarrow A^{k-1} \rightarrow A^{k} \rightarrow A^{k+1} \rightarrow \cdots,
$$

where it is implicit that the arrows represent instances of the differential $d$.

A complex $A^{\bullet}$ is said to be exact at an index $k$ if $\mathrm{H}^{k} A^{\bullet}=0$, or equivalently:

$$
\operatorname{ker} d^{k}: A^{k} \rightarrow A^{k+1}=\operatorname{im} d^{k-1}: A^{k-1} \rightarrow A^{k} .
$$

It is said to be exact if it is exact at each index where the cohomology group is defined.

Example 2.1 The complex $A^{0} \rightarrow A^{1} \rightarrow 0$ is exact iff the first arrow is surjective.

The complex $0 \rightarrow A^{0} \rightarrow A^{1}$ is exact iff the second arrow is injective.

If $B \subseteq A$ we have an exact sequence $0 \rightarrow B \rightarrow A \rightarrow A / B \rightarrow 0$.

If $C=A \oplus B$ we also have an exact sequence of the form $0 \rightarrow A \rightarrow C \rightarrow$ $B \rightarrow 0$.

Remark 2.1 If we have an exact sequence of finite dimensional spaces $0 \rightarrow A \rightarrow B \rightarrow C \rightarrow 0$ we have $\operatorname{dim} A-\operatorname{dim} B+\operatorname{dim} C=0$ and any two terms of the sequence determine the third term up to isomorphism. A similar result holds for longer exact sequences.

If $A^{\bullet}$ and $B^{\bullet}$ are two complexes, a morphism of complexes $f^{\bullet}: A^{\bullet} \rightarrow$ $B^{\bullet}$, is a sequence of linear operators $f^{k}: A^{k} \rightarrow B^{k}$ such that the following diagrams commute:

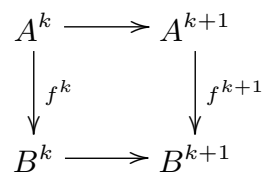

Proposition 2.1 A morphism of complexes $f^{\bullet}: A^{\bullet} \rightarrow B^{\bullet}$ induces a sequence of linear maps $\mathrm{H}^{k} f^{\bullet}: \mathrm{H}^{k} A^{\bullet} \rightarrow \mathrm{H}^{k} B^{\bullet}:$ to the class of $u \in \operatorname{ker} d^{k}$ : $A^{k} \rightarrow A^{k+1}$ we associate the class of $f^{k} u$.

- Proof: This is based on two facts:

If $u \in A^{k}$ satisfies $d^{k} u=0$ then $d^{k} f^{k} u=f^{k+1} d^{k} u=0$.

Also if $u=d^{k-1} v$ for some $v \in A^{k-1}$ then $f^{k} u=f^{k} d^{k-1} v=d^{k-1} f^{k-1} v$ with $f^{k-1} v \in B^{k-1}$, hence the class of $u$ determines the class of $f^{k} u$.

We have that $\mathrm{H}^{k}\left(f^{\bullet} g^{\bullet}\right)=\left(\mathrm{H}^{k} f^{\bullet}\right)\left(\mathrm{H}^{k} g^{\bullet}\right)$ and $\mathrm{H}^{k}(\mathrm{id})=\mathrm{id}$.

As an illustration of the above concepts we notice:

Remark 2.2 Suppose $A^{\bullet}$ is a complex and, for each $k, B^{k}$ is a subspace of $A^{k}$ such that the differential $A^{k} \rightarrow A^{k+1}$ induces differentials $B^{k} \rightarrow B^{k+1}$ by restriction. Suppose furthermore we have projections $p^{k}: A^{k} \rightarrow B^{k}$ commuting with the differential. Then $p^{\bullet}$ induces surjections in cohomology. 
- Proof: If $u \in B^{k}$ satisfies $d u=0$ then $u \in A^{k}$ satisfies $d u=0$ and $p u=u$.

Given two morphisms of complexes $f^{\bullet}, g^{\bullet}: A^{\bullet} \rightarrow B^{\bullet}$, a homotopy operator from $f^{\bullet}$ to $g^{\bullet}$ is a family $h^{\bullet}$ of linear operators $h^{k}: A^{k} \rightarrow B^{k-1}$ such that:

$$
g^{k}-f^{k}=h^{k+1} d^{k}+d^{k-1} h^{k} .
$$

Proposition 2.2 If $f^{\bullet}$ and $g^{\bullet}$ are homotopic in the sense that a homotopy operator exists from one to the other, they induce the same maps $\mathrm{H}^{k} A^{\bullet} \rightarrow \mathrm{H}^{k} B^{\bullet}$.

- Proof: Pick $u \in A^{k}$ such that $d u=0$. Then $g u-f u=d h u$ so $g u$ and $f u$ determine the same class modulo $\mathrm{d} B^{k-1}$ in $\mathrm{H}^{k} B^{\bullet}$.

Recall that if $\alpha: A \rightarrow A^{\prime}$ then coker $\alpha=A^{\prime} /(\alpha A)$.

We will use the snake lemma:

Lemma 2.1 Suppose we have a commuting diagram:

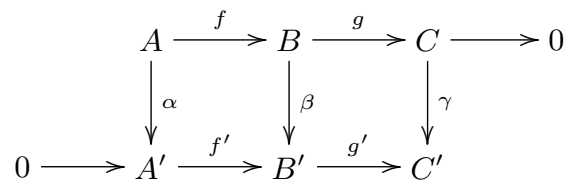

We suppose each row is exact. Then we have a well-defined morphism:

$$
\delta: \operatorname{ker} \gamma \rightarrow \operatorname{coker} \alpha
$$

It is defined on any $w \in C$ such that $\gamma w=0$, by taking first any reciprocal $v$ of $w$ by $g$, then mapping $v$ to $v^{\prime}=\beta v$, then taking the reciprocal $u^{\prime}$ of $v^{\prime}$ by $f^{\prime}$ and finally considering the equivalence class of $u^{\prime}$ modulo $\alpha A$.

- Proof: Pick $w \in C$ such that $d w=0$ and choose $v \in B$ such that $g v=w$.

We have $g^{\prime} \beta v=\alpha g v=d w=0$, so there is $u^{\prime} \in A^{\prime}$ such that $f^{\prime} u^{\prime}=\beta v$. If we had chosen $\hat{v} \in B$ instead of $v$, we would have obtained say $\hat{u}^{\prime} \in A^{\prime}$. We have $g(\hat{v}-v)=0$ so $\hat{v}-v=f u$ for a $u \in A$.

Then $f^{\prime}\left(\hat{u}^{\prime}-u^{\prime}\right)=\beta(\hat{v}-v)=\beta f u=f^{\prime} \alpha u$, so $\hat{u}^{\prime}-u^{\prime}=\alpha u$.

Theorem 2.3 Suppose we are given three complexes $A^{\bullet}, B^{\bullet}$ and $C^{\bullet}$, and morphisms of complexes $f^{\bullet}: A^{\bullet} \rightarrow B^{\bullet}$ and $g^{\bullet}: B^{\bullet} \rightarrow C^{\bullet}$, providing for each $k$ a short exact sequence:

$$
0 \rightarrow A^{k} \rightarrow B^{k} \rightarrow C^{k} \rightarrow 0 .
$$

Then one can construct a long exact sequence linking the cohomology groups:

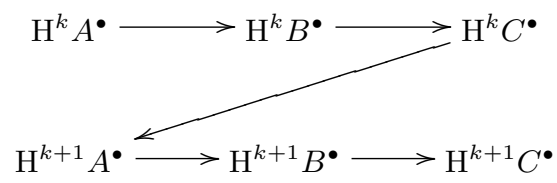

Here the first, second, fourth and fifth arrows are the natural ones and the third, diagonal, one - called connecting morphism and usually denoted $\delta^{k}$ - is constructed by the snake lemma.

- Proof: We denote by $f^{k}$ and $g^{k}$ the maps $f^{k}: A^{k} \rightarrow B^{k}$ and $g^{k}: B^{k} \rightarrow$ $C^{k}$. The proof consists of a series of straightforward verifications, to the effect that we have a complex and that it is exact. 
Exactness at $\mathrm{H}^{k} B^{\bullet}$ :

Pick $v^{k} \in B^{k}$ such that $\mathrm{d} v^{k}=0$ and $g^{k} v^{k}=d w^{k-1}$ for some $w^{k-1} \in C^{k-1}$. Choose $v^{k-1} \in B^{k-1}$ such that $g^{k-1} v^{k-1}=w^{k-1}$.

We have $g^{k}\left(v^{k}-d v^{k-1}\right)=d w^{k-1}-d g^{k-1} v^{k-1}=0$.

Put $v^{k}-d v^{k-1}=f^{k} u^{k}$ for some $u^{k} \in A^{k}$.

We have $f^{k+1} d u^{k}=d f^{k} u^{k}=d\left(v^{k}-d v^{k-1}\right)=0$, hence $d u^{k}=0$.

Construction of $\delta^{k}$ :

Recall that given $w^{k} \in C^{k}$ such that $d w^{k}=0$ we choose any $v^{k} \in B^{k}$ such that $g^{k} v^{k}=w^{k}$. Then we take the $u^{k+1} \in A^{k+1}$ such that $f^{k+1} u^{k+1}=$ $d v^{k}$, and consider the class of $u^{k+1}$.

If $w^{k}=d w^{k-1}$ for some $w^{k-1} \in C^{k-1}$ we have $w^{k-1}=g^{k-1} v^{k-1}$ with $v^{k-1} \in B^{k-1}$. Then $g^{k} d v^{k-1}=d g^{k-1} v^{k-1}=d w^{k-1}=w^{k}$, so we may suppose $v^{k}=d v^{k-1}$. Then $d v^{k}=d d v^{k-1}=0$ so $u_{k+1}=0$.

Remark also that $f^{k+2} d u^{k+1}=d f^{k+1} u^{k+1}=d d v^{k}=0$, so $d u^{k+1}=0$.

Concerning $\delta^{k} \mathrm{H}^{k} g^{\bullet}$ :

If $w^{k}=g^{k} v^{k}$ for an $v^{k}$ such that $d v^{k}=0$, then $f^{k+1} u^{k+1}=d v^{k}=0$ so $u^{k+1}=0$, hence the composition is zero.

If $u^{k+1}=d u^{k}$ for some $u^{k} \in A^{k}$, then $g^{k}\left(v^{k}-f^{k} u^{k}\right)=w^{k}$ and $d\left(v^{k}-\right.$ $\left.f^{k} u^{k}\right)=d v^{k}-f^{k+1} d u^{k}=d v^{k}-f^{k+1} u^{k+1}=0$.

Concerning $\mathrm{H}^{k+1} f^{\bullet} \delta^{k}$ :

We have $f^{k+1} u^{k+1}=d v^{k}$ hence the composition is zero.

If $f^{k+1} u^{k+1}=d v^{k}$ for some $v^{k} \in B^{k}$, then $d g^{k} v^{k}=g^{k+1} d v^{k}=g^{k+1} f^{k+1} u^{k+1}=$ 0 and $\delta^{k} g^{k} v^{k}=u^{k+1}$.

Such proofs are called diagram chasing.

Example 2.2 If we are given a morphism of complexes $g^{\bullet}: B^{\bullet} \rightarrow C^{\bullet}$ consisting of surjections and define $A^{k}$ to be the kernel of $g^{k}$, we remark that $A^{\bullet}$ is a subcomplex of $B^{\bullet}$ called the kernel complex, which has trivial cohomology if and only if $g^{\bullet}$ induces isomorphisms in cohomology $\mathrm{H}^{k} B^{\bullet} \rightarrow$ $\mathrm{H}^{k} C^{\bullet}$.

Example 2.3 Suppose $A^{\bullet}$ is a complex. Define $Z^{k}, B^{k} \subseteq A^{k}$ by:

$$
\begin{aligned}
Z^{k} & =\left\{u \in A^{k}: d u=0\right\}, \\
B^{k} & =\left\{d u: u \in A^{k-1}\right\} .
\end{aligned}
$$

These are both subcomplexes of $A^{\bullet}$ and their differentials are the 0 morphisms. We have short exact sequences:

$$
0 \rightarrow Z^{k} \rightarrow A^{k} \rightarrow B^{k+1} \rightarrow 0
$$

Moreover the arrows here are morphisms of complexes. We thus get a long exact sequence:

$$
\cdots B^{k} \rightarrow Z^{k} \rightarrow \mathrm{H}^{k} A^{\bullet} \rightarrow B^{k+1} \rightarrow Z^{k+1} \cdots
$$

It turns out that the connecting morphism is the inclusion $B^{k} \subseteq Z^{k}$ which is injective, so we get exact sequences:

$$
0 \rightarrow B^{k} \rightarrow Z^{k} \rightarrow \mathrm{H}^{k} A^{\bullet} \rightarrow 0 .
$$

which confirm, if need be, that:

$$
\mathrm{H}^{k} A^{\bullet} \simeq Z^{k} / B^{k} .
$$

Remark 2.3 Suppose we have an exact sequence:

$$
A^{1} \stackrel{\phi}{\longrightarrow} A^{2} \longrightarrow A^{3} \longrightarrow A^{4} \stackrel{\psi}{\longrightarrow} A^{5} \text {. }
$$


Then we have a short exact sequence:

$$
0 \rightarrow \operatorname{coker} \phi \rightarrow A_{3} \rightarrow \operatorname{ker} \psi \rightarrow 0 .
$$

In particular, full knowledge of the connecting morphism associated with a short exact sequence of complexes determines the cohomology of the middle complex.

The construction of the connecting morphism $\delta$ has the following property. Suppose that the above situation holds for complexes $A_{0}^{\bullet}, B_{0}^{\bullet}$ and $C_{0}^{\bullet}$ (with $f_{0}^{\bullet}$ and $g_{0}^{\bullet}$ ) and also for $A_{1}^{\bullet}, B_{1}^{\bullet}$ and $C_{1}^{\bullet}$ (with $f_{1}^{\bullet}$ and $g_{1}^{\bullet}$ ). Suppose furthermore that we have morphisms of complexes $\alpha^{\bullet}: A_{0}^{\bullet} \rightarrow A_{1}^{\bullet}$, $\beta^{\bullet}: B_{0}^{\bullet} \rightarrow B_{1}^{\bullet}$ and $\gamma^{\bullet}: C_{0}^{\bullet} \rightarrow C_{1}^{\bullet}$ intertwining $f_{1}^{\bullet}$ with $f_{0}^{\bullet}$ and $g_{1}^{\bullet}$ with $g_{0}^{\bullet}$. Explicitly we have commuting diagrams:

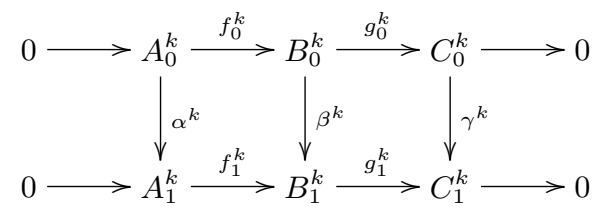

Then the following diagram commutes:

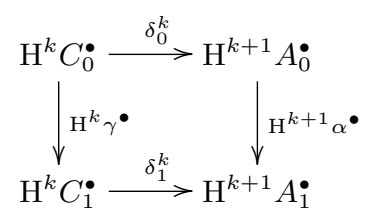

(This property is a functoriality property of $\delta$.)

The following result is known as the five lemma.

Lemma 2.2 Suppose we have a commuting diagram:

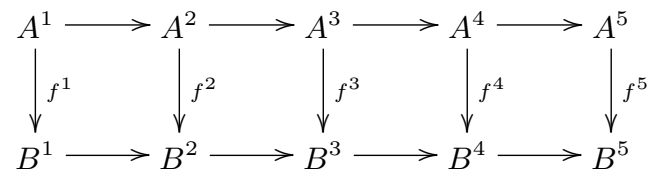

where each row is exact. If $f^{1}, f^{2}, f^{4}$ and $f^{5}$ are isomorphisms, then $f^{3}$ is also an isomorphism.

- Proof: We let $u^{i}$ denote elements of $A^{i}$ and $v^{i}$ those of $B^{i}$. All horizontal arrows are denoted $d$. We prove:

If $f^{1}$ is surjective and $f^{2}, f^{4}$ are injective, then $f^{3}$ is injective:

Suppose $f^{3} u^{3}=0$. Then $f^{4} d u^{3}=d f^{3} u^{3}=0$ hence $d u^{3}=0$. Put $u^{3}=d u^{2}$.

We have $d f^{2} u^{2}=f^{3} d u^{2}=f^{3} u^{3}=0$. Put $f^{2} u^{2}=d v^{1}$.

We have $f^{2} d u^{1}=d f^{1} u^{1}=d y^{1}=f^{2} u^{2}$. Hence $d u^{1}=u^{2}$.

We have $u^{3}=d d u^{1}=0$.

If $f^{5}$ is injective and $f^{2}, f^{4}$ are surjective, then $f^{3}$ is surjective:

Pick $v^{3}$. Write $d v^{3}=f^{4} u^{4}$.

We have $f^{5} d u^{4}=d f^{4} u^{4}=d d v^{3}=0$ hence $d u^{4}=0$. Put $u^{4}=d u^{3}$.

We have $d v^{3}=f^{4} u^{4}=f^{4} d u^{3}=d f^{3} u^{3}$ hence $v^{3}-f^{3} u^{3}=d v^{2}$ and $v^{2}=f^{2} u^{2}$.

We have $v^{3}=f^{3} u^{3}-d v^{2}=f^{3} u^{3}-d f^{2} u^{2}=f^{3}\left(u^{3}-d u^{2}\right)$. 
Multilinear algebra and tensor products Differential forms (which will be defined later) involve multilinear maps on the tangent spaces of a manifold. The tensor products of multilinear maps provides a convenient tool for studying such maps. Moreover differential forms on a product manifold have a special structure best described in terms of tensor products of spaces.

If $U$ is a vector space $U^{\star}$ denotes its dual. If $U$ and $V$ are vectorspaces $\mathrm{L}(U, V)$ is the space of linear maps $U \rightarrow V$. If $U, V$ and $W$ are vectorspaces $\mathrm{B}(U, V ; W)$ is the space of bilinear maps $U \times V \rightarrow W$. Recall that if $b: U \times V \rightarrow W$ is bilinear, $b(U, V)$ designates the subspace of $W$ generated by elements of the form $b(u, v)$, with $u \in U$ and $v \in V$.

Let $U, V$ be vectorspaces. We say that $(W, \otimes)$ is a tensorproduct of $U$ and $V$, if $W$ is a vectorspace and $\otimes: U \times V \rightarrow W$ is bilinear and for any $W^{\prime}$ and any $b: U \times V \rightarrow W^{\prime}$ there is a unique $\beta: W \rightarrow W^{\prime}$ such that for all $u \in U$ and all $v \in V$ :

$$
b(u, v)=\beta(u \otimes v) .
$$

Thus tensorproducts, when they exist, are unique up to canonical isomorphism.

Remark $2.4(W, \otimes)$ is a tensorproduct of $U$ and $V$ iff the following two conditions hold. First, for any free families $\left(u_{i}\right)_{i \in I}$ in $U$ and $\left(v_{j}\right)_{j \in J}$ in $V$, the family $\left(u_{i} \otimes v_{j}\right)_{(i, j) \in I \times J}$ is free in $W$. Second, the vectorspace $W$ is generated by elements of the form $u \otimes v$ for $u \in U$ and $v \in V$.

Let $U, V$ be vectorspaces. Put $W=\mathrm{B}(U, V)^{\star}$ and define, for $u \in U$, $v \in V$ and $b \in \mathrm{B}(U, V)$ :

$$
(u \otimes v)(b)=b(u, v) .
$$

Then $\otimes: U \times V \rightarrow W$ is bilinear and $(U \otimes V, \otimes)$ is a tensorproduct of $U$ and $V$. Thus tensorproducts exist. Unless otherwise specified this is the tensorproduct we will use.

When $U, V$ and $W$ are vectorspaces and a tensor product of $U$ and $V$ is chosen, we have a canonical isomorphism:

$$
\mathrm{L}(U \otimes V, W) \rightarrow \mathrm{B}(U, V ; W) .
$$

Let $V$ be a vectorspace. $\mathrm{L}^{k}(V)$ denotes the space of $k$-linear maps $V^{k} \rightarrow \mathbb{R}$. In particular $\mathrm{L}^{1}(V)$ is the dual of $V$, also denoted $V^{\star}$.

If $u \in \mathrm{L}^{k}(V)$ and $v \in \mathrm{L}^{l}(V)$ then $u \otimes v \in \mathrm{L}^{k+l}(V)$ is defined by:

$$
u \otimes v\left(\xi_{1}, \cdots, \xi_{k+l}\right)=u\left(\xi_{1}, \cdots, \xi_{k}\right) v\left(\xi_{k+1}, \cdots, \xi_{k+l}\right) .
$$

Lemma 2.3 If $\left(u_{i}\right)_{i \in I}$ is free in $\mathrm{L}^{k}(V)$ and $\left(v_{j}\right)_{j \in J}$ is free in $\mathrm{L}^{l}(V)$ then the family $\left(u_{i} \otimes v_{j}\right)_{(i, j) \in I \times J}$ is free in $\mathrm{L}^{k+l}(V)$.

Comparing dimensions we find that if $V$ is finite-dimensional $\left(\mathrm{L}^{k+l}(V), \otimes\right)$ is a tensorproduct of $\mathrm{L}^{k}(V)$ and $\mathrm{L}^{l}(V)$.

Suppose $f_{i}: U_{i} \rightarrow V_{i}$ for $i=0,1$. Define $f_{0} \otimes f_{1}: U_{0} \otimes U_{1} \rightarrow V_{0} \otimes V_{1}$ by:

$$
\left(f_{0} \otimes f_{1}\right)\left(u_{0} \otimes u_{1}\right)=\left(f_{0} u_{0}\right) \otimes\left(f_{1} u_{1}\right)
$$

It is called the Kronecker product of $f_{0}$ and $f_{1}$. When all spaces are finite dimensional, $\mathrm{L}\left(U_{0} \otimes U_{1}, V_{0} \otimes V_{1}\right)$ equipped with the Kronecker product, is indeed a tensor product of $\mathrm{L}\left(U_{0}, V_{0}\right)$ and $\mathrm{L}\left(U_{1}, V_{1}\right)$ 
Remark 2.5 If $b: U \times V \rightarrow Z$ is bilinear any $w \in b(U, V)$ can be written $w=\sum_{i j} \lambda_{i j} b\left(u_{i}, v_{j}\right)$ with $\left(u_{i}\right)$ and $\left(v_{j}\right)$ free.

The following canonical isomorphisms are useful. If $U \subseteq V$ we have for any $W$ :

$$
(V \otimes W) /(U \otimes W) \simeq(V / U) \otimes W .
$$

If $U_{i} \subseteq V_{i}$ for $i=0,1$ we have:

$$
\left(V_{0} \oplus V_{1}\right) /\left(U_{0} \oplus U_{1}\right) \simeq\left(V_{0} / U_{0}\right) \oplus\left(V_{1} / U_{1}\right) .
$$

An element $u \in \mathrm{L}^{k}(V)$ is alternating iff for any permutation $\sigma \in \mathfrak{S}_{k}$ :

$$
u\left(\xi_{\sigma_{1}}, \cdots, \xi_{\sigma_{k}}\right)=\epsilon(\sigma) u\left(\xi_{1}, \cdots, \xi_{k}\right) .
$$

The subspace of $\mathrm{L}^{k}(V)$ consisting of alternating forms is denoted $\mathrm{L}_{a}^{k}(V)$. The canonical projection in $\mathrm{L}^{k}(V)$ onto $\mathrm{L}_{a}^{k}(V)$ is denoted $\alpha_{k}$.

$$
\left(\alpha_{k} u\right)\left(\xi_{1}, \cdots, \xi_{k}\right)=\frac{1}{k !} \sum_{\sigma} \epsilon(\sigma) u\left(\xi_{\sigma_{1}}, \cdots, \xi_{\sigma_{k}}\right),
$$

where the sum extends over the set of permutations of $\{1, \cdots, k\}$.

The wedge product of $u \in \mathrm{L}_{a}^{k}(V)$ and $v \in \mathrm{L}_{a}^{l}(V)$ is $u \wedge v \in \mathrm{L}_{a}^{k+l}(V)$ defined by:

$$
u \wedge v=\frac{(k+l) !}{k ! l !} \alpha_{k+l}(u \otimes v) .
$$

The wedge product is bilinear and associative. We have, for $u_{i} \in V^{\star}$ :

$$
\left(u_{1} \wedge \cdots \wedge u_{k}\right)\left(\xi_{1}, \cdots, \xi_{k}\right)=\sum_{\sigma} \epsilon(\sigma) u_{1}\left(\xi_{\sigma_{1}}\right) \cdots u_{k}\left(\xi_{\sigma_{k}}\right) .
$$

Moreover if $u \in \mathrm{L}_{a}^{k}(V)$ and $v \in \mathrm{L}_{a}^{l}(V)$ :

$$
v \wedge u=(-1)^{k l} u \wedge v \text {. }
$$

This property is known as graded commutativity.

Suppose $V$ is $n$-dimensional. If $\left(e_{i}\right)_{1 \leq i \leq n}$ is a basis of $V^{\star}$ then the following family is a basis of $A_{k}(V)$ :

$$
e_{i_{1}} \wedge \cdots \wedge e_{i_{k}} \text { for } 1 \leq i_{1}<\cdots<i_{k} \leq n
$$

It may be regarded as being indexed by the set of subsets of $\{1, \cdots, n\}$ with cardinality $k$. In particular:

$$
\operatorname{dim} \mathrm{L}_{a}^{k}(V)=\left(\begin{array}{l}
n \\
k
\end{array}\right) .
$$

If $F: V_{0} \rightarrow V_{1}$ is linear and $u \in \mathrm{L}^{k}\left(V_{1}\right)$ then $F^{\star} u \in \mathrm{L}^{k}\left(V_{0}\right)$ is defined by:

$$
F^{\star} u\left(\xi_{1}, \cdots, \xi_{k}\right)=u\left(F \xi_{1}, \cdots, F \xi_{k}\right) .
$$

If $u \in \mathrm{L}_{a}^{k}\left(V_{1}\right)$ then $F^{\star} u \in \mathrm{L}_{a}^{k}\left(V_{0}\right)$.

Suppose $V=V_{0} \oplus V_{1}$. Let $P_{0}$ be the projection onto $V_{0}$ with kernel $V_{1}$ and $P_{1}$ be the projection onto $V_{1}$ with kernel $V_{0}$. Then the spaces $P_{0}^{\star} \mathrm{L}_{a}^{k_{0}}\left(V_{0}\right) \otimes P_{1}^{\star} \mathrm{L}_{a}^{k_{1}}\left(V_{1}\right)$ are in direct sum.

Proposition 2.4 With a slight abuse of notations, the following map is an isomorphism:

$$
\alpha_{k}: \bigoplus_{k_{0}+k_{1}=k} \mathrm{~L}_{a}^{k_{0}}\left(V_{0}\right) \otimes \mathrm{L}_{a}^{k_{1}}\left(V_{1}\right) \rightarrow \mathrm{L}_{a}^{k}(V) .
$$


- Proof: Prove injectivity and remark that, by the formula:

$$
\sum_{k_{0}+k_{1}=k}\left(\begin{array}{l}
n_{0} \\
k_{0}
\end{array}\right)\left(\begin{array}{l}
n_{1} \\
k_{1}
\end{array}\right)=\left(\begin{array}{l}
n \\
k
\end{array}\right),
$$

where $n=n_{0}+n_{1}$, the dimensions are equal.

A scalar product on $V$ is a $g \in \mathrm{L}^{2}(V)$ which is symmetric and positive definite. It induces a scalar product on $V^{\star}$ by requiring that $u \mapsto g(u, \cdot)$ is an isometry. In other words if $l, l^{\prime} \in V^{\star}$ are represented as $l=g(u, \cdot)$ and $l^{\prime}=g\left(u^{\prime}, \cdot\right)$ then we define $g\left(l, l^{\prime}\right)=g\left(u, u^{\prime}\right)$. There is a unique scalar product on multilinear forms such that:

$$
g\left(u_{1} \otimes \cdots \otimes u_{k}, v_{1} \otimes \cdots \otimes v_{k}\right)=g\left(u_{1}, v_{1}\right) \cdots g\left(u_{k}, v_{k}\right) .
$$

If we restrict this scalar product to alternating forms we notice:

$$
g\left(u_{1} \wedge \cdots \wedge u_{k}, v_{1} \wedge \cdots \wedge v_{k}\right)=k ! \sum_{\sigma} \epsilon(\sigma) g\left(u_{1}, v_{\sigma_{1}}\right) \cdots g\left(u_{k}, v_{\sigma_{k}}\right) .
$$

Actually one scales this scalar product and define:

$$
g\left(u_{1} \wedge \cdots \wedge u_{k}, v_{1} \wedge \cdots \wedge v_{k}\right)=\operatorname{det} g\left(u_{i}, v_{j}\right) .
$$

With this scaling, if $\left(e_{i}\right)_{1 \leq i \leq n}$ is an orthonormal basis of $V^{\star}$, then the family:

$$
e_{i_{1}} \wedge \cdots \wedge e_{i_{k}} \text { for } 1 \leq i_{1}<\cdots<i_{k} \leq n,
$$

indexed by subsets of $\{1, \cdots, n\}$ of cardinality $k$, is orthonormal in $\mathrm{L}_{a}^{k}(V)$.

A scalar product and an orientation of $V$ give rise to a volume form $\omega$, namely the determinant in any orthonormal oriented basis. Suppose $\operatorname{dim}(V)=n$. The Hodge star is the map $\star: \mathrm{L}_{a}^{k}(V) \rightarrow \mathrm{L}_{a}^{n-k}(V)$ uniquely determined by:

$$
u \wedge \star v=g(u, v) \omega .
$$

If $\left(e_{i}\right)_{1 \leq i \leq n}$ is an orthonormal basis of $V^{\star}$ then for any set $i_{1}<\cdots<i_{k}$ with complement $j_{1}<\cdots<j_{n-k}$ we have:

$$
\star\left(e_{i_{1}} \wedge \cdots \wedge e_{i_{k}}\right)=\epsilon(I, J) e_{j_{1}} \wedge \cdots \wedge e_{j_{n-k}},
$$

where $\epsilon(I, J)$ is the sign of the permutation $\left(i_{1}, \cdots, i_{k}, j_{1}, \cdots, j_{n-k}\right)$.

One checks that: $\star \star= \pm$ id and $\star^{\prime}= \pm \star$

We now turn to the interplay between tensorproducts and homological algebra.

A graded vectorspace is a sequence $A^{\bullet}=\left(A^{k}\right)_{k \in \mathbb{Z}}$ of vector spaces, identified with the space:

$$
A=\bigoplus_{k} A^{k} .
$$

Thus a complex is a particular kind of graded vectorspace, as well as the family of its cohomology groups. The map H taking a complex to its cohomology sequence is a functor from complexes to graded vectorspaces.

If $F$ and $G$ are graded vectorspaces, their tensor product is by definition the graded vectorspace defined by:

$$
(F \otimes G)^{l}=\bigoplus_{k} F^{k} \otimes G^{l-k},
$$

and

$$
(u \otimes v)^{l}=\sum_{k} u^{k} \otimes u^{l-k}
$$


If $F$ and $G$ are both complexes (i.e. they are equipped with differentials) then $F \otimes G$ is a complex when equipped with the differential defined on $F^{k} \otimes G^{l-k}$ by:

$$
d(u \otimes v)=(d u) \otimes v+(-1)^{k} u \otimes(d v) .
$$

If $A$ is graded we introduce the map $\sigma: A \rightarrow A$ defined on $A^{k}$ by $\sigma u=(-1)^{k} u$. Thus $\sigma \sigma=\operatorname{id}_{A}$ and if $A$ is equipped with a differential $d$ we notice that $d \sigma=-\sigma d$.

With this definition we can write for any $u \in F$ and $v \in G$ :

$$
d(u \otimes v)=(d u) \otimes v+(\sigma u) \otimes(d v) .
$$

This gives a proof that this is indeed a differential:

$$
\begin{aligned}
d d(u \otimes v) & =d((d u) \otimes v+(\sigma u) \otimes(d v)), \\
& =d d u \otimes v+(\sigma d u+d \sigma u) \otimes(d v)+(\sigma \sigma u) \otimes(d d v), \\
& =0 .
\end{aligned}
$$

Remark 2.6 Suppose $F_{i}$ and $G_{i}$ are complexes for $i=0,1$ and $f_{i}: F_{i} \rightarrow$ $G_{i}$ are morphisms of complexes. Then the Kronecker product $f_{0} \otimes f_{1}$ is a morphism of complexes $F_{0} \otimes F_{1} \rightarrow G_{0} \otimes G_{1}$.

The following is the algebraic Kunneth theorem.

Theorem 2.5 We have a canonical isomorphism:

$$
\mathrm{H}(F) \otimes \mathrm{H}(G) \simeq \mathrm{H}(F \otimes G) .
$$

- Proof: We will use the following remark. Suppose that $A$ is a graded vectorspace equipped with the 0 differential. Then $A \otimes G$ has the differential:

$$
d: A^{k} \otimes G^{l-k} \rightarrow A^{k} \otimes G^{l+1-k} \text { with } u \otimes v \mapsto(-1)^{k} u \otimes d v .
$$

Thus we get:

$$
\mathrm{H}^{l}(A \otimes G) \simeq(A \otimes \mathrm{H}(G))^{l} .
$$

Return now to the case of $F \otimes G$. Recalling the construction of Example 2.3 , we denote by $B^{k}, Z^{k} \subseteq F^{k}$ the image and kernel of the differential of $F$. We have short exact sequences:

$$
0 \rightarrow Z^{k} \rightarrow F^{k} \rightarrow B^{k+1} \rightarrow 0 .
$$

Tensoring with the identity of $G^{l-k}$ we get short exact sequences, for each $l$ :

$$
0 \rightarrow \bigoplus_{k} Z^{k} \otimes G^{l-k} \rightarrow \bigoplus_{k} F^{k} \otimes G^{l-k} \rightarrow \bigoplus_{k} B^{k+1} \otimes G^{l-k} \rightarrow 0
$$

Thus we have a short exact sequence of complexes:

$$
0 \rightarrow(Z \otimes G)^{\bullet} \rightarrow(F \otimes G)^{\bullet} \rightarrow(B \otimes G)^{\bullet+1} \rightarrow 0
$$

It gives a long exact sequence:

$\mathrm{H}^{l}(B \otimes G) \rightarrow \mathrm{H}^{l}(Z \otimes G) \rightarrow \mathrm{H}^{l}(F \otimes G) \rightarrow \mathrm{H}^{l+1}(B \otimes G) \rightarrow \mathrm{H}^{l+1}(Z \otimes G)$.

Using the above remark we get:

$(B \otimes \mathrm{H}(G))^{l} \rightarrow(Z \otimes \mathrm{H}(G))^{l} \rightarrow \mathrm{H}^{l}(F \otimes G) \rightarrow(B \otimes \mathrm{H}(G))^{l+1} \rightarrow(Z \otimes \mathrm{H}(G))^{l+1}$ 
The connecting morphism, appearing here as the first and last arrow, is the Kronecker product of the inclusion $B^{k} \subseteq Z^{k}$ with the identity of $\mathrm{H}(G)$. It is injective. Thus we get short exact sequences:

$$
0 \rightarrow(B \otimes \mathrm{H}(G))^{l} \rightarrow(Z \otimes \mathrm{H}(G))^{l} \rightarrow \mathrm{H}^{l}(F \otimes G) \rightarrow 0 .
$$

But we have:

$$
(Z \otimes \mathrm{H}(G))^{l} /(B \otimes \mathrm{H}(G))^{l} \simeq \bigoplus_{k}\left(Z^{k} / B^{k}\right) \otimes \mathrm{H}^{l-k}(G) .
$$

Thus we get:

$$
\mathrm{H}^{l}(F \otimes G) \simeq(\mathrm{H}(F) \otimes \mathrm{H}(G))^{l},
$$

which is what we want.

\subsection{Differential geometry}

In this section we outline continuous geometry (differential forms), discrete geometry (cellular complexes) and the relation between the two. For details see [44], [61], [54] and [13].

Continuous geometry Let $M$ be a topological space. A chart on $M$ is a continuous map $F: U \rightarrow M$ where $U$ is open in some normed vector (half-) space, such that $F(U)$ is open in $M$ and the induced map $U \rightarrow F(U)$ is a homeomorphism. An atlas on $M$ is a family of charts $F_{i}: U_{i} \rightarrow M$ such that the sets $F_{i}\left(U_{i}\right)$ cover $M$.

A manifold is a topological space equipped with an atlas. Suppose $\left(F_{i}: U_{i} \rightarrow M\right)$ is an atlas. Put $V_{i}=F\left(U_{i}\right)$ and $V_{i j}=V_{i} \cap V_{j}$. The atlas is said to be differentiable if the maps:

$$
F_{j i}=F_{j}^{-1} F_{i}: F_{i}^{-1}\left(V_{i j}\right) \rightarrow F_{j}^{-1}\left(V_{i j}\right),
$$

are differentiable. These maps are called transition maps.

Put $V_{i j k}=V_{i} \cap V_{j} \cap V_{k}$. Then, on $F_{i}^{-1}\left(V_{i j k}\right)$, we have well defined maps satisfying:

$$
F_{k i}=F_{k j} F_{j i}
$$

Notice also that:

$$
F_{i i}=\mathrm{id} .
$$

The manifold $M$ can be identified with the disjoint union $\cup_{i \in I}\{i\} \times U_{i}$ quotiented by the finest relation such that:

$$
(i, x) \sim\left(j, F_{j i}(x)\right),
$$

whenever $F_{j i}$ is defined on $x$. Explicitly an identification is well-defined by:

$$
(i, x) \mapsto F_{i}(x) .
$$

The tangent manifold is the disjoint union $\cup_{i \in I}\{i\} \times U_{i} \times V$ quotiented by the relation:

$$
(i, x, \xi) \sim\left(j, F_{j i}(x), \mathrm{D} F_{j i}(x) \xi\right)
$$

The canonical projection $\pi: \mathrm{T} M \rightarrow M$ is well defined by:

$$
\pi(i, x, \xi)=(i, x) .
$$

The tangent space of $M$ at $x$ is $\mathrm{T}_{x} M=\pi^{-1}(x)$. It is a vector space.

Remark 2.7 Tangent map. 
The space of smooth functions $M \rightarrow \mathbb{R}$ is denoted $\mathcal{F}(M)$.

A vectorfield on $M$ is a smooth map $X: M \rightarrow \mathrm{T} M$ such that $\pi X=$ $\operatorname{id}_{M}$. In other words it is an object which to any $x \in M$ associates an element $X_{x} \in \mathrm{T}_{x} M$. The space of vectorfields on $M$ is denoted $\mathcal{V}(M)$.

A differential form of degree $k$ on a manifold $M$ is an object which to every point $x$ associates an element $u_{x} \in \mathrm{L}_{a}^{k}\left(\mathrm{~T}_{x} M\right)$. Such an object is also called a $k$-form. The space of smooth $k$-forms is denoted $\Omega^{k}(M)$. If $u \in \Omega^{k}(M)$ and $v \in \Omega^{l}(M)$ then their wedge product is $u \wedge v \in \Omega^{k+l}(M)$ defined pointwise. Explicitly:

$(u \wedge v)_{x}\left(\xi_{1}, \cdots, \xi_{k+l}\right)=\frac{1}{k ! l !} \sum_{\sigma} \epsilon(\sigma) u_{x}\left(\xi_{\sigma_{1}}, \cdots, \xi_{\sigma_{k}}\right) v_{x}\left(\xi_{\sigma_{k+1}}, \cdots, \xi_{\sigma_{k+l}}\right)$,

where the sum extends over all permutations.

If $F: M \rightarrow N$ and $u \in \Omega^{k}(N)$, the pull-back of $u$ to $N$ is denoted $F^{\star} u$ and defined by:

$$
\left(F^{\star} u\right)_{x}\left(\xi_{1}, \cdots, \xi_{k}\right)=u_{F(x)}\left(\mathrm{D} F(x) \xi_{1}, \cdots, \mathrm{D} F(x) \xi_{k}\right) .
$$

In other words:

$$
\left(F^{\star} u\right)_{x}=\mathrm{D} F(x)^{\star} u_{F(x)} .
$$

If $F_{0}: M_{0} \rightarrow M_{1}$ and $F_{1}: M_{1} \rightarrow M_{2}$ then $\left(F_{1} F_{0}\right)^{\star}=F_{0}^{\star} F_{1}^{\star}$.

Let $U$ be open in a vector space $V$. An element $u \in \Omega^{k}(U)$ can be considered as a map $U \rightarrow \mathrm{L}_{a}^{k}(V)$. Its total (or Fréchet) derivative is a map:

$$
\mathrm{D} u: U \rightarrow \mathrm{L}\left(V, \mathrm{~L}_{a}^{k}(V)\right) \subseteq \mathrm{L}^{k+1}(V) .
$$

The exterior derivative $\mathrm{d}: \Omega^{k}(U) \rightarrow \Omega^{k+1}(U)$ is defined by:

$$
(\mathrm{d} u)_{x}\left(\xi_{0}, \cdots, \xi_{k}\right)=\sum_{i=0}^{k}(-1)^{i}(\mathrm{D} u)_{x}\left(\xi_{i}, \xi_{0}, \cdots, \hat{\xi}_{i}, \cdots, \xi_{k}\right) .
$$

In other words:

$$
\mathrm{d} u=(k+1) \alpha_{k+1}(\mathrm{D} u),
$$

where $\alpha_{k}$ is the canonical antisymmetrizing projector on $k$-linear forms.

One checks that:

Lemma 2.4 If $F: U_{0} \rightarrow U_{1}$ is a map:

$$
\mathrm{d} F^{\star} u=F^{\star} \mathrm{d} u .
$$

- Proof: We have:

$$
\left(F^{\star} u\right)_{x}\left(\xi_{1}, \cdots, \xi_{k}\right)=u_{F(x)}\left(\mathrm{D} F(x) \xi_{1}, \cdots, \mathrm{D} F(x) \xi_{k}\right) .
$$

Hence:

$$
\begin{aligned}
& \mathrm{D}\left(F^{\star} u\right)_{x}\left(\xi_{0}, \xi_{1}, \cdots, \xi_{k}\right) \\
= & (\mathrm{D} u)_{F(x)}\left(\mathrm{D} F(x) \xi_{0}, \mathrm{D} F(x) \xi_{1}, \cdots, \mathrm{D} F(x) \xi_{k}\right)+ \\
& \sum_{i=1}^{k} u_{F(x)}\left(\mathrm{D} F(x) \xi_{1}, \cdots, \mathrm{D}^{2} F(x)\left(\xi_{0}, \xi_{i}\right), \cdots, \mathrm{D} F(x) \xi_{k}\right)
\end{aligned}
$$

The sum disappears upon anti-symmetrization, and the first term corresponds to the pull-back of $F^{\star} \mathrm{d} u$.

From this it follows that the exterior derivative can be defined on manifolds, yielding a map $\mathrm{d}: \Omega^{k}(M) \rightarrow \Omega^{k+1}(M)$. It has the following properties: 
Proposition 2.6 For any map $F: M_{0} \rightarrow M_{1}$, any $u \in \Omega^{k}\left(M_{1}\right)$ we have:

$$
\mathrm{d} F^{\star} u=F^{\star} \mathrm{d} u \text {. }
$$

For any $u \in \Omega^{k}(M)$ and $v \in \Omega^{l}(M)$ (Leibniz formula):

$$
\mathrm{d}(u \wedge v)=(\mathrm{d} u) \wedge v+(-1)^{k} u \wedge(\mathrm{d} v) .
$$

Moreover $\mathrm{d}^{2}=0$. In other words we have a complex:

$$
\cdots \rightarrow \Omega^{k}(M) \rightarrow \Omega^{k+1}(M) \rightarrow \cdots
$$

- Proof: The first property follows from Lemma 2.4. We likewise prove the two others in a chart over $U \subseteq V$.

We have:

$$
\begin{aligned}
\mathrm{d}(u \wedge v) & =(k+l+1) \alpha_{k+l+1} \mathrm{D}(u \wedge v), \\
& =\frac{(k+l+1) !}{k ! l !} \alpha_{k+l+1} \mathrm{D}(u \otimes v), \\
& =\frac{(k+l+1) !}{k ! l !} \alpha_{k+l+1}((\mathrm{D} u) \otimes v+\tau(u \otimes \mathrm{D} v)),
\end{aligned}
$$

where $\tau$ is the cyclic permutation of the variables represented by:

$$
(0, \cdots, k+l) \rightarrow(1, \cdots, k, 0, k+1, \cdots, k+l) .
$$

The sign of this permutation is $(-1)^{k}$. This gives the second identity.

We have:

$$
\begin{aligned}
\operatorname{dd} u & =\alpha_{k+2} \mathrm{D}\left(\alpha_{k+1} \mathrm{D} u\right) \\
& =\alpha_{k+2} \mathrm{D}^{2} u
\end{aligned}
$$

Since $\mathrm{D}^{2} u$ is symmetric in its first two arguments this gives the third identity.

Remark 2.8 The exterior derivative is uniquely determined by properties (34) and (35) together with its action on $\Omega^{0}(M)$. Indeed elements of the form $u_{0} \mathrm{~d} u_{1} \wedge \cdots \wedge \mathrm{d} u_{k}$ with $u_{i} \in \Omega^{0}(M)$ generate $\Omega^{k}(M)$.

If $u$ is a differential $k$-form and $X$ a vectorfield, the contraction of $u$ by $X$ is $i_{X} u$ defined by:

$$
\left(i_{X} u\right)_{x}\left(\xi_{2}, \cdots, \xi_{k}\right)=u_{x}\left(X(x), \xi_{2}, \cdots \xi_{k}\right) .
$$

We shall define integration of $n$-forms on $n$-dimensional oriented manifolds with boundary. A non-degenerate smooth $n$-form is called a volume form. The local model of such a manifold is:

$$
\mathbb{R}_{+}^{n}=\left\{\left(x_{1}, \cdots, x_{n}\right) \in \mathbb{R}^{h}: x_{1} \leq 0\right\}
$$

Let $\omega$ denote the canonical volume form on $\mathbb{R}^{n}$. Let $U_{0}$ and $U_{1}$ be two open subsets of $\mathbb{R}_{+}^{n}$ and $F: U_{0} \rightarrow U_{1}$ an orientation preserving diffeomorphism. The determinant of $\mathrm{D} F(x)$ in orthonormal oriented bases (the Jacobian) is denoted Jac $F(x)$. For $i=0,1$ let $u_{i}=f_{i} \omega$ be a differential $n$-form on $U_{i}$, with $f_{i}$ a function on $U_{i}$. We suppose that $u_{0}=F^{\star} u_{1}$. Then $f_{0}=f_{1} \circ F$ Jac $F$ hence $f_{0}$ is integrable iff $f_{1}$ is integrable and in this case:

$$
\int_{U_{1}} f_{1}=\int_{U_{0}} f_{0} .
$$


An atlas is said to be orientation-preserving if all transition maps are orientation-preserving. A manifold is orientable if it has an orientationpreserving atlas. Two oriented atlases are orientation-equivalent if the union is oriented. If a manifold with $N$ connected components is orientable this equivalence relation on oriented atlases has $2^{N}$ equivalence classes. An orientation of the manifold is the choice of such an equivalence class, and an oriented atlas is an atlas in this equivalence class.

Let $M$ be a compact manifold of dimension $n$ which is oriented. Let $\left(F_{i}: U_{i} \rightarrow M\right)$ be a countable oriented atlas, and $\left(\phi_{i}\right)$ be a subordinated partition of unity. Let $u \in \Omega^{n}(M)$. Put $u_{i}=F_{i}^{\star} u$ and let $u_{i}=f_{i} \omega$. We say that $u$ is integrable if the $f_{i}$ are measurable and:

$$
\sum_{i} \int_{U_{i}}\left(\phi_{i} \circ F_{i}\right)\left|f_{i}\right|<+\infty
$$

If $u$ is integrable we put:

$$
\int u=\sum_{i} \int_{U_{i}}\left(\phi_{i} \circ F_{i}\right) f_{i} .
$$

One checks that the above notions of integrability and integral are independent of the choice of (countable oriented) atlas and partition of unity. We notice that for any chart $F: U \rightarrow M$ and any $u \in \Omega^{n}(M)$ if we define $f$ by $F^{\star} u=f \omega$, we have:

$$
\int_{F(U)} u=\int_{U} f
$$

The charts $F_{i}: U_{i} \rightarrow M$ are such that:

$$
\forall x \in U_{i} \quad F_{i}(x) \in \partial M \Longleftrightarrow x_{1}=0 .
$$

The maps $\left(\left.F_{i}\right|_{x_{1}=0}\right)$ constitute an orientation-preserving atlas of $\partial M$. It determines the induced orientation of $\partial M$.

Let $M$ be manifold with boundary $\partial M$ oriented outwardly by the orientation of $M$. If $\omega$ is a positively oriented volume form on $M$, and $X$ is a field on $\partial M$ of vectors in TM (a section of the injection $\partial M \rightarrow \mathrm{T} M$ ) pointing out of $M$, then $i_{X} \omega$ is a positively oriented volume form on $\partial M$.

The following result is known as Stokes' theorem.

Theorem 2.7 For any compact manifold $M$, any $u \in \Omega^{n-1}(M)$ which is continuous up to the boundary, and whose exterior derivative is integrable:

$$
\int_{M} \mathrm{~d} u=\int_{\partial M} u
$$

The following result concerning the cohomology of contractible domains is known as Poincaré's lemma. Recall that a domain is contractible is the identity map is homotopic to a constant map. Using Lie derivatives and Cartan's formula, one shows that a homotopy $t \mapsto f_{t}$ between maps $f_{0}$ and $f_{1}$ gives a homotopy operator (in the algebraic sense) between the pullbacks $f_{0}^{\star}$ and $f_{1}^{\star}$.

Lemma 2.5 If $M$ is a contractible domain then $\mathrm{H}^{k}(M)=0$ for $k \geq 1$ (and $\mathrm{H}^{0}(M)$ consists of the constant functions).

Suppose $M$ is a three dimensional manifold equipped with a Riemannian metric $g$. Given a vectorfield $u \in \mathcal{V}(M)$ one can associate with it the two forms $\Psi^{1} u=g(u, \cdot) \in \Omega^{1}(X)$ and $\Psi^{2} u=\omega(u, \cdots) \in \Omega^{2}(X)$. A 
function $u \in \mathcal{F}(M)$ can be associated with itself considered as an element $\Psi^{0} u=u \in \Omega^{0}(M)$ and with the form $\Psi^{3} u=u \omega \in \Omega^{3}(M)$. One then has the commuting diagram:

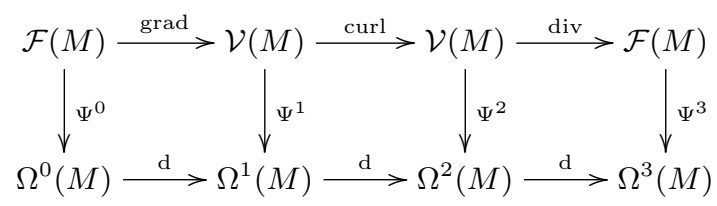

where the vertical arrows are isomorphisms. Through this correspondence problems expressed in terms of vector fields can be translated into problems expressed with differential forms.

Discrete geometry A $k$-simplex is a set with $k+1$ elements $(k \geq 0)$; its elements are called vertices. The standard simplex is $[k]=\{0, \cdots, k\}$. Its geometric realization is $\Delta_{k}$ defined by:

$$
\Delta_{k}=\left\{\left(x_{0}, \cdots, x_{k}\right) \in \mathbb{R}^{k+1}: x_{i} \geq 0 \text { and } x_{0}+\cdots+x_{k}=1\right\} .
$$

The vertices of $\Delta_{k}$ are the standard basis vectors $\left(e_{i}^{k}\right)_{i \in[k]}$ of $\mathbb{R}^{k+1}$. If $\sigma:\left[k^{\prime}\right] \rightarrow[k]$ is a map, there is a unique affine map $\Delta_{k^{\prime}} \rightarrow \Delta_{k}$ sending $e_{i}^{k^{\prime}}$ to $e_{\sigma(i)}^{k}$.

A simplicial complex $\mathcal{T}$ is a set of finite non-empty sets (simplices), such that for any $T$ in $\mathcal{T}$, any nonempty subset of $T$ is also in $\mathcal{T}$. Such a subset of $T$ is called a face. It is proper when it is different from $T$. A $k$-simplex is said to be $k$-dimensional. For each $k$ we let $\mathcal{T}^{k}$ denote the subset of $\mathcal{T}$ consisting of $k$-dimensional simplices.

A simplicial complex determines a topological space by gluing together spaces $\Delta_{k}$ using the connectivity of $\mathcal{T}$. More precisely $|\mathcal{T}|$ denotes the set of maps $x: \mathcal{T}^{0} \rightarrow[0,1]$ such that $x$ has support in a $T \in \mathcal{T}$ (i.e. there exists a $T \in \mathcal{T}$ such that $x$ is non-zero only on the vertices of $T$ ) and moreover:

$$
\sum_{\alpha} x(\alpha)=1
$$

For any simplex $T \in \mathcal{T}$ we denote by $|T|$ the set of $x \in|\mathcal{T}|$ with support in $T$. Notice that for any bijection $\alpha:[k] \rightarrow T$ we have a bijection $|\alpha|: \Delta_{k} \rightarrow|T|$. The topology of $|\mathcal{T}|$ is defined by the property that $F \subseteq|\mathcal{T}|$ is closed iff $F \cap|T|$ is closed for each $T$, when $|T|$ is equipped with the topology inherited from some $\Delta_{k}$.

For simplices, orientation can be defined combinatorially. For a $k$ simplex $T$, we consider the equivalence relation on bijections $\alpha:[k] \rightarrow T$ defined by $\alpha \sim \alpha^{\prime}$ iff $\alpha^{-1} \alpha^{\prime}$ is an even permutation of [k]. This equivalence relation has two equivalence classes when $k \geq 1$ and a combinatorial orientation of $T$ is a choice of one of the equivalence classes. The standard simplex $[k]$ has a canonical combinatorial orientation, namely the equivalence class of the identity map, the set of even permutations. We say that the elements of the combinatorial orientation of $T$ are the orientation preserving maps $[k] \rightarrow T$. A combinatorial orientation of $T$ determines an orientation of the manifold $|T|$ by requiring that the induced map $|\alpha|: \Delta_{k} \rightarrow|T|$ be orientation preserving for any orientation preserving map $\alpha:[k] \rightarrow T$. The chosen orientation of $\Delta_{k}$ is the one such that $\mathrm{d} x_{1} \wedge \cdots \wedge \mathrm{d} x_{k}$ is positive, or equivalently the one it obtains as the boundary of the simplex:

$$
\left\{\left(x_{0}, \cdots, x_{k}\right): x_{i} \geq 0 \text { and } \sum x_{i} \leq 1\right\} .
$$


equipped with the orientation of $\mathbb{R}^{k+1}$.

Let $T$ be a $(k+1)$-simplex and $T^{\prime}$ a $k$-face, equipped with combinatorial orientations. Choose an orientation preserving $\alpha:[k] \rightarrow T^{\prime}$. Then $\epsilon\left(T, T^{\prime}\right)=1$ iff the bijection $[k+1] \rightarrow T$ defined by:

$$
0 \mapsto T \backslash T^{\prime} \text { and } i+1 \mapsto \alpha_{i},
$$

is orientation preserving.

Example 2.4 Let $M$ be a topological space and $\left(U_{i}\right)_{i \in I}$ be an open cover. The nerve of this cover is the simplicial complex consisting of the following subsets of $I$ :

$$
\left\{\alpha_{0}, \cdots, \alpha_{k}\right\} \subseteq I: U_{\alpha_{0}} \cap \cdots \cap U_{\alpha_{k}} \neq \emptyset .
$$

In what follows we shall frequently identify $T$ with $|T|$.

Let $M$ denote a compact metric space.

To us, a $k$-dimensional cell in $M$ is a closed subset $T$ of $M$ which is homeomorphic by a bi-Lipschitz map to the closed unit ball of $\mathbb{R}^{k}$. If a cell $T$ is both $k$ and $l$ dimensional then $k=l$. We denote by $\partial T$ its boundary, the image of the unit sphere in $\mathbb{R}^{k}$ by the chosen homeomorphism. Different homeomorphisms to the ball give the same boundary. The interior of $T$ is by definition $T \backslash \partial T$ (it is open in $T$ but not necessarily in $M$ ). A cellular complex on $M$ is a finite set $\mathcal{T}$ of cells in $M$ such that the following conditions hold:

- The union of all cells in $\mathcal{T}$ is $M$.

- Distinct cells in $\mathcal{T}$ have disjoint interiors.

- The intersection of two cells in $\mathcal{T}$ is a union of cells in $\mathcal{T}$.

- The boundary of any cell in $\mathcal{T}$ is a union of cells in $\mathcal{T}$.

- The topology of $M$ is such that a subset $F$ of $M$ is closed iff $F \cap T$ is closed in $T$ for any cell $T$ in $\mathcal{T}$.

A simplicial complex is a cellular complex in which the intersection of any two cells is a cell (not just a union of cells) and such that the boundary of any cell is split into subcells in the same way as the boundary of a reference simplex is usually split into subsimplexes. The reference simplex of dimension $k$ is:

$$
\left\{\left(x_{0}, \cdots, x_{k}\right) \in \mathbb{R}^{k+1}: x_{i} \geq 0 \text { and } \sum_{i} x_{i}=1\right\},
$$

and its subsimplexes are defined by equations $x_{i}=0$ for all $i \in J$, where $J \subseteq\{0, \cdots, k\}$.

We put for each $k \in \mathbb{N}$ :

$$
\mathcal{T}^{k}=\{T \in \mathcal{T}: \operatorname{dim} T=k\} .
$$

The boundary of any cell $T$ of $\mathcal{T}$ can be naturally equipped with a cellular complex, namely:

$$
\left\{T^{\prime} \in \mathcal{T}: T^{\prime} \subseteq T, T^{\prime} \neq T\right\} .
$$

A refinement of a cellular complex $\mathcal{T}$ on $M$ is a cellular complex $\mathcal{T}^{\prime}$ on $M$ such that each element of $\mathcal{T}$ is the union of elements of $\mathcal{T}^{\prime}$. We will be particularly interested in simplicial refinements of a cellular complex.

A cellular subcomplex of a cellular complex $\mathcal{T}$ on $M$, is a cellular complex $\mathcal{T}^{\prime}$ on some closed part $M^{\prime}$ of $M$ such that $\mathcal{T}^{\prime} \subseteq \mathcal{T}$. For instance 
we have seen the the boundary of any cell $T$ in $\mathcal{T}$ can be equipped with a cellular complex which is a subcomplex of $\mathcal{T}$.

Fix a cellular complex $(M, \mathcal{T})$. In the following we suppose that for each $T \in \mathcal{T}$ of dimension $\geq 1$, the manifold $|T|$ has been oriented. Relative orientations, also called incidence numbers, are defined as follows:

For any edge $T$ its vertices are ordered by the orientation of $T$, call them $\alpha_{0}$ and $\alpha_{1}$ in increasing order and define $\epsilon\left(T,\left\{\alpha_{1}\right\}\right)=1$ and $\epsilon\left(T,\left\{\alpha_{0}\right\}\right)=$ -1 . This definition is extended to higher dimensional cells as follows. Fix $k \geq 1$. Given $T \in \mathcal{T}^{k+1}$ and $T^{\prime} \in \mathcal{T}^{k}$, if $T^{\prime} \subseteq T$ we define $\epsilon\left(T, T^{\prime}\right)=1$ if $T^{\prime}$ is outward oriented compared with $T$ and $\epsilon\left(T, T^{\prime}\right)=-1$ if it is inward oriented. For all $T, T^{\prime} \in \mathcal{T}$ not covered by this definition we put $\epsilon\left(T, T^{\prime}\right)=0$.

For each $k$ let $C^{k}(\mathcal{T})$ denote the set of maps $c: \mathcal{T}^{k} \rightarrow \mathbb{R}$. Such maps are called $k$-cochains. The coboundary operator $\delta: C^{k}(\mathcal{T}) \rightarrow C^{k+1}(\mathcal{T})$ is defined by:

$$
(\delta c)_{T}=\sum_{T^{\prime}} \epsilon\left(T, T^{\prime}\right) c_{T^{\prime}}
$$

In other words it's the operator whose canonical matrix is $\epsilon$. We remark that the coefficients in the sum can be non-zero only when $T^{\prime} \in \partial T \cap \mathcal{T}^{k}$.

Lemma 2.6 We have that $\delta \delta=0$ as a $\operatorname{map} C^{k}(\mathcal{T}) \rightarrow C^{k+2}(\mathcal{T})$.

- Proof: Pick $u \in C^{k}(\mathcal{T})$. Pick $T \in \mathcal{T}^{k+2}$. Let $T^{\prime \prime}$ be a $k$-face of $T$; it is shared by two $(k+1)$-faces of $T$ denoted $T_{0}^{\prime}$ and $T_{1}^{\prime}$.

Suppose $T_{0}^{\prime}$ and $T_{1}^{\prime}$ have the same orientation compared with $T$. Then $T^{\prime \prime}$ is oppositely oriented relative to $T_{0}^{\prime}$ and $T_{1}^{\prime}$. If $T_{0}^{\prime}$ and $T_{1}^{\prime}$ have opposite orientations compared with $T$ then $T^{\prime \prime}$ has the same orientation relative to $T_{0}^{\prime}$ and $T_{1}^{\prime}$.

In both cases the contribution of $u_{T^{\prime \prime}}$ to $(\delta \delta u)_{T}$ is 0 .

In other words the family $C^{\bullet}(\mathcal{T})$ is a complex, called the cochain complex and represented by:

$$
0 \rightarrow C^{0}(\mathcal{T}) \rightarrow C^{1}(\mathcal{T}) \rightarrow C^{2}(\mathcal{T}) \rightarrow \cdots
$$

Suppose $\mathcal{T}$ is a cellular complex equipped with an orientation (of the cells) and $\mathcal{T}^{\prime}$ is a cellular refinement also equipped with an orientation (for instance the same complex but with different orientations). For each cell $T \in \mathcal{T}^{k}$ and each $T^{\prime} \in \mathcal{T}^{\prime k}$ define $\iota\left(T, T^{\prime}\right)= \pm 1$ if $\left|T^{\prime}\right| \subseteq|T|$ and they have the same/different orientation, and $\iota\left(T, T^{\prime}\right)=0$ in all other cases.

Proposition 2.8 The map $\iota: C^{\bullet}\left(\mathcal{T}^{\prime}\right) \rightarrow C^{\bullet}(\mathcal{T})$ defined by:

$$
(\iota u)_{T}=\sum_{T^{\prime}} \iota\left(T, T^{\prime}\right) u_{T^{\prime}},
$$

is a morphism of complexes.

- Proof: We view $\iota^{k}$ as a matrix indexed by $\mathcal{T}^{k} \times \mathcal{T}^{\prime k}$. We also consider the compositions $\delta \iota$ and $\iota \delta^{\prime}$ as matrices. Pick now $T \in \mathcal{T}^{k+1}$ and $T^{\prime} \in \mathcal{T}^{\prime k}$.

Suppose $\left|T^{\prime}\right|$ is not a subset of $|T|$. Any $(k+1)$-cell of $\mathcal{T}^{\prime}$ containing $\left|T^{\prime}\right|$ is not included in $|T|$ so $\left(\iota \delta^{\prime}\right)_{T T^{\prime}}=0$. Any $k$-cell of $\mathcal{T}$ containing $\left|T^{\prime}\right|$ is not included in $|T|$ so $(\delta \iota)_{T T^{\prime}}=0$.

Suppose $\left|T^{\prime}\right|$ is a subset of $|T|$, but not of $|\partial T|$. Then $\left|T^{\prime}\right|$ is not included in any $k$-cell of $\mathcal{T}$ so $(\delta \iota)_{T T^{\prime}}=0$. On the other hand $\left|T^{\prime}\right|$ is included in exactly two $(k+1)$-cells of $\mathcal{T}^{\prime}$ inside $|T|$. If they have equal orientations compared with $|T|$, they induce opposite orientations on $\left|T^{\prime}\right|$, and vice-versa. Hence $\left(\iota \delta^{\prime}\right)_{T T^{\prime}}$ is the sum of two opposite terms. 
Suppose $\left|T^{\prime}\right|$ is a subset of $|\partial T|$ and let $T_{1}$ be the $k$-cell of $\mathcal{T}$ containing $\left|T^{\prime}\right|$ and $T_{1}^{\prime}$ the $(k+1)$-cell of $\mathcal{T}^{\prime}$ included in $|T|$ and containing $\left|T^{\prime}\right|$. We have:

$$
\left(\iota \delta^{\prime}\right)_{T T^{\prime}}=\iota\left(T, T_{1}^{\prime}\right) \epsilon^{\prime}\left(T_{1}^{\prime}, T^{\prime}\right),
$$

and:

$$
(\delta \iota)_{T T^{\prime}}=\epsilon\left(T, T_{1}\right) \iota\left(T_{1}, T^{\prime}\right) .
$$

These terms are both equal to the relative orientation of $\left|T^{\prime}\right|$ with respect to $|T|$. This completes the proof.

Relating continuous and discrete geometry Let $M$ be a manifold and $\mathcal{T}$ a cellular complex on $M$. The complex $C^{\bullet}(\mathcal{T})$ can be related to $\Omega^{\bullet}(M)$ as follows. For each $T \in \mathcal{T}^{k}$ let $\mu_{T}$ be the linear form on $\Omega^{k}$ defined by:

$$
\mu_{T}:\left.u \mapsto \int_{T} u\right|_{T}
$$

For each $k$ we denote by $\mu^{k}: \Omega^{k}(M) \rightarrow C^{k}(\mathcal{T})$ the map defined by:

$$
\mu^{k}: u \mapsto\left(\mu_{T} u\right)_{\left(T \in \mathcal{T}^{k}\right)} .
$$

In the setting of finite element computations, the linear forms $\mu_{T}$ are called degrees of freedom.

Proposition 2.9 For each $k$ the following diagram commutes:

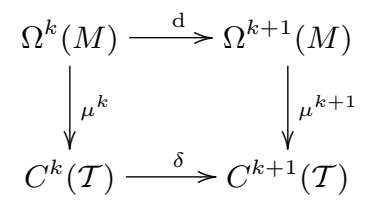

- Proof: This is an application of Stokes theorem.

It is easy to check that the maps $\mu^{k}: \Omega^{k}(M) \rightarrow C^{k}(\mathcal{T})$ are onto so that the above diagram uniquely determines $\delta: C^{k}(\mathcal{T}) \rightarrow C^{k+1}(\mathcal{T})$.

Suppose $\mathcal{T}$ is a simplicial complex, with oriented simplices. We suppose that $\left(\lambda_{\alpha}\right)_{\alpha \in \mathcal{T}^{0}}$ is a partition of unity such that the support of $\lambda_{\alpha}$ is in the closed star of $\alpha$ (the union of the simplices containing the vertex $\alpha)$. Thus if $\alpha \notin T$ we have $\left.\lambda_{\alpha}\right|_{T}=0$.

If $T \in \mathcal{T}^{k}$, and $\alpha:[k] \rightarrow T$ is orientation preserving, we define a $k$-form $\lambda_{T}$ attached to $T$ by:

$$
\lambda_{T}=k ! \sum_{i=0}^{k}(-1)^{i} \lambda_{\alpha_{i}} \mathrm{~d} \lambda_{\alpha_{0}} \wedge \cdots\left(\mathrm{d} \lambda_{\alpha_{i}}\right)^{\wedge} \cdots \mathrm{d} \lambda_{\alpha_{k}} .
$$

The following notation will be used. For any functions $u_{0}, \cdots, u_{k}$ we put:

$$
\begin{aligned}
{\left[u_{0}, \cdots, u_{k}\right] } & =\sum_{i=0}^{k}(-1)^{i} u_{i} \mathrm{~d} u_{0} \wedge \cdots\left(\mathrm{d} u_{i}\right)^{\wedge} \cdots \wedge \mathrm{d} u_{k} \\
& =\frac{1}{k !} \sum_{\sigma} \epsilon(\sigma) u_{\sigma_{0}} \mathrm{~d} u_{\sigma_{1}} \wedge \cdots \wedge \mathrm{d} u_{\sigma_{k}}
\end{aligned}
$$

where the sum extends over permutations of $[k]$. In particular the definition of $\lambda_{T}$ does not depend on the choice of $\alpha$ as long as it is orientation 
preserving. Remark also that if two of the $u_{i}$ are equal one obtains 0 . Suppose that $\sigma:[k] \rightarrow[k]$ is a map. If $\sigma$ is not bijective we have:

$$
\left[u_{\sigma_{0}}, \cdots, u_{\sigma_{k}}\right]=0 .
$$

If $\sigma$ is bijective then:

$$
\left[u_{\sigma_{0}}, \cdots, u_{\sigma_{k}}\right]=\epsilon(\sigma)\left[u_{0}, \cdots, u_{k}\right] .
$$

Remark furthermore that if $\alpha:[k] \rightarrow \mathcal{T}^{0}$ is a map whose range is not a simplex, then:

$$
\left[\lambda_{\alpha_{0}}, \cdots, \lambda_{\alpha_{k}}\right]=0
$$

Proposition 2.10 For any $T, T^{\prime} \in \mathcal{T}^{k}$ we have $\mu_{T^{\prime}} \lambda_{T}=\delta_{T T^{\prime}}$.

- Proof: If $T^{\prime} \neq T$, there is $\alpha \in T \backslash T^{\prime}$. Since $\left.\lambda_{\alpha}\right|_{T^{\prime}}=0$ we have $\left.\lambda_{T}\right|_{T^{\prime}}=0$, hence $\mu_{T^{\prime}} \lambda_{T}=0$.

To compute $\mu_{T} \lambda_{T}$ we choose an orientation preserving $\alpha:[k] \rightarrow T$ and notice that on $T$ :

$$
\begin{aligned}
\lambda_{T} & =k ! \mathrm{d} \lambda_{\alpha_{1}} \wedge \cdots \wedge \lambda_{\alpha_{k}}, \\
& =\epsilon\left(T, T^{\prime}\right) \mathrm{d} \lambda_{T^{\prime}},
\end{aligned}
$$

where $T^{\prime}=\left\{\alpha_{1}, \cdots, \alpha_{k}\right\}$ is the face of $T$ opposite to $\alpha_{0}$. The identity then follows by an induction argument using Stokes theorem.

We define:

$$
\Lambda^{k}(\mathcal{T})=\operatorname{span}\left\{\lambda_{T}: T \in \mathcal{T}^{k}\right\}
$$

By the preceding proposition, $\left(\lambda_{T}\right)_{T \in \mathcal{T}^{k}}$ is a basis of $\Lambda^{k}(\mathcal{T})$.

Proposition 2.11 The exterior derivative d maps $\Lambda^{k}(\mathcal{T})$ into $\Lambda^{k+1}(\mathcal{T})$ and the matrix of $\mathrm{d}: \Lambda^{k}(\mathcal{T}) \rightarrow \Lambda^{k+1}(\mathcal{T})$ in the bases $\left(\lambda_{T^{\prime}}\right)_{T^{\prime} \in \mathcal{T}^{k}} \rightarrow$ $\left(\lambda_{T}\right)_{T \in \mathcal{T}^{k+1}}$ has entry $\epsilon\left(T, T^{\prime}\right)$ at the indices $\left(T, T^{\prime}\right) \in \mathcal{T}^{k+1} \times \mathcal{T}^{k}$.

- Proof: Choose $T^{\prime} \in \mathcal{T}^{k}$. Let $\alpha:[k] \rightarrow T^{\prime}$ be orientation preserving. First we remark that:

$$
\begin{aligned}
& \mathrm{d} \lambda_{T^{\prime}} \\
= & (k+1) ! \mathrm{d} \lambda_{\alpha_{0}} \wedge \cdots \wedge \mathrm{d} \lambda_{\alpha_{k}}, \\
= & (k+1) !\left(\sum_{\alpha \notin T^{\prime}} \lambda_{\alpha} \mathrm{d} \lambda_{\alpha_{0}} \wedge \cdots \wedge \mathrm{d} \lambda_{\alpha_{k}}+\sum_{i \in[k]} \lambda_{\alpha_{i}} \mathrm{~d} \lambda_{\alpha_{0}} \wedge \cdots \wedge \mathrm{d} \lambda_{\alpha_{k}}\right) .
\end{aligned}
$$

The last sum can be replaced by:

$$
-\sum_{i \in[k]} \lambda_{\alpha_{i}} \mathrm{~d} \lambda_{\alpha_{0}} \wedge \cdots\left(\mathrm{d} \sum_{\alpha \notin T^{\prime}} \lambda_{\alpha}\right)_{\text {at } i} \cdots \wedge \mathrm{d} \lambda_{\alpha_{k}} .
$$

Moving $\mathrm{d} \lambda_{\alpha}$ to the front we get:

$$
\begin{aligned}
\mathrm{d} \lambda_{T^{\prime}} & =(k+1) ! \sum_{\alpha \notin T^{\prime}}\left(\lambda_{\alpha} \mathrm{d} \lambda_{\alpha_{0}} \wedge \cdots \wedge \mathrm{d} \lambda_{\alpha_{k}}-\mathrm{d} \lambda_{\alpha} \wedge\left[\lambda_{\alpha_{0}}, \cdots, \lambda_{\alpha_{k}}\right]\right), \\
& =(k+1) ! \sum_{\alpha \notin T^{\prime}}\left[\lambda_{\alpha}, \lambda_{\alpha_{0}}, \cdots, \lambda_{\alpha_{k}}\right] .
\end{aligned}
$$

In this sum an $\alpha$-term is nonzero only if $\left\{\alpha, \alpha_{0}, \cdots, \alpha_{k}\right\} \in \mathcal{T}^{k+1}$. By definition of the incidence numbers we have:

$$
\mathrm{d} \lambda_{T^{\prime}}=\sum_{T \in \mathcal{T}^{k+1}} \epsilon\left(T, T^{\prime}\right) \lambda_{T} .
$$


This completes the proof.

Thus $\Lambda^{\bullet}(\mathcal{T})$ is a subcomplex of $\Omega^{\bullet}(M)$ which is isomorphic (not just in cohomology) to the cochain complex of $\mathcal{T}$.

We can define projections:

$$
I^{k}: \Omega^{k}(M) \rightarrow \Lambda^{k}(\mathcal{T}),
$$

by requiring that $\mu^{k} I^{k} u=\mu^{k} u$. These projections commute with the exterior derivative.

Corollary 2.12 Let a compact manifold $M$ be equipped with a simplicial complex $\mathcal{T}$. Then the De Rham map induces surjections in cohomology.

- Proof: We apply Remark 2.2 to the present situation.

Corollary 2.13 Let $M$ be a starshaped domain, triangulated by a simplicial complex $\mathcal{T}$. Then the simplicial cohomology is trivial in dimension $k>0$ and has dimension 1 for $k=0$.

- Proof: We know that $\mathrm{H}^{k} \Omega^{\bullet}(M)$ is trivial for $k>0$ by Poincaré's lemma. Combined with Corollary 2.12 this gives the result.

A significant strengthening of Corollary 2.12 is the following theorem of De Rham:

Theorem 2.14 Let a compact manifold $M$ be equipped with a simplicial complex. The the De Rham map induces isomorphisms in cohomology.

- Proof: See [61], [54] or [13].

\subsection{Finite elements on cellular complexes}

The notion of finite element system we shall consider here was introduced in [23]. We develop it in particular in the direction of tensor products.

Finite element systems Let $\mathcal{T}$ be a cellular complex. For each $k$ and each $T \in \mathcal{T}$ we suppose we are given a space $A^{k}(T)$ of differential $k$-forms on $T$ such that the exterior derivative induces maps $\mathrm{d}: A^{k}(T) \rightarrow A^{k+1}(T)$ and if $T^{\prime} \subseteq T$ the inclusion map $i:\left|T^{\prime}\right| \rightarrow|T|$ induces a map $i^{\star}: A^{k}(T) \rightarrow$ $A^{k}\left(T^{\prime}\right)$. We call such a family of spaces a finite element system. For any subcomplex $\mathcal{T}^{\prime}$ of $\mathcal{T}$ we define $A^{k}\left(\mathcal{T}^{\prime}\right)$ to consist of families $\left(u_{T}\right)_{T \in \mathcal{T}^{\prime}}$ such that whenever $T^{\prime} \subseteq T$ and $i:\left|T^{\prime}\right| \rightarrow|T|$ is the inclusion map, we have $i^{\star} u_{T}=u_{T^{\prime}}$ (equivalently $\left.u_{T}\right|_{T^{\prime}}=u_{T^{\prime}}$ ).

We say that the finite element system $A$ is compatible when in addition the following conditions hold:

- Cohomology. We require that the following sequence be exact:

$$
0 \rightarrow \mathbb{R} \rightarrow A^{0}(T) \rightarrow A^{1}(T) \rightarrow \cdots \rightarrow A^{\operatorname{dim}(T)}(T) \rightarrow 0 .
$$

- Restrictions. If we denote by $\rho:|\partial T| \rightarrow|T|$ the inclusion map, we require that the pullback $\rho^{\star}: A^{k}(T) \rightarrow A^{k}(\partial T)$ (the restriction to the boundary) be surjective. In other words compatible forms on the boundary of a cell can be extended to the interior.

The following notation will be handy. For any cellular subcomplex $\mathcal{T}^{\prime}$ of $\mathcal{T}$ we set:

$$
A^{\bullet}\left(\mathcal{T}^{\prime}\right)=\bigoplus_{k} A^{k}\left(\mathcal{T}^{\prime}\right)
$$

and remark that it is indeed a complex when equipped with the exterior derivative. 
We denote by $A_{0}^{k}(T)$ the kernel of $\rho^{\star}: A^{k}(T) \rightarrow A^{k}(\partial T)$. The following result is about the dimension of $A^{k}(\mathcal{T})$ when the spaces $A^{k}(T)$ are finite dimensional.

Proposition 2.15 Suppose a finite element system has the extension property. Then:

$$
\operatorname{dim} A^{k}(\mathcal{T})=\sum_{T \in \mathcal{T}} \operatorname{dim} A_{0}^{k}(T) .
$$

- Proof: For any $l$, recall that $\mathcal{T}^{l}$ is the subset of $\mathcal{T}$ of $l$-dimensional cells. We let $\mathcal{T}^{(l)}$ denote the subset of cells of dimension at most $l$ - the so-called $l$-skeleton of $\mathcal{T}$.

For $l \geq 1$, let $\rho^{l}$ denote the restriction $A^{k}\left(\mathcal{T}^{(l)}\right) \rightarrow A^{k}\left(\mathcal{T}^{(l-1)}\right)$. Then $\rho^{l}$ is a surjection and its kernel can be identified with:

$$
\operatorname{ker} \rho^{l}=\bigoplus_{T \in \mathcal{T}^{l}} A_{0}^{k}(T) .
$$

Thus we get:

$$
\operatorname{dim} A^{k}\left(\mathcal{T}^{(l)}\right)=\operatorname{dim} A^{k}\left(\mathcal{T}^{(l-1)}\right)+\sum_{T \in \mathcal{T}^{l}} \operatorname{dim} A_{0}^{k}(T) .
$$

Repeating this identity for $l$ ranging from the maximal dimension of cells of $\mathcal{T}$ down to 1 we get the claimed identity.

Remark 2.9 We remark that if the maps $A^{k}(T) \rightarrow A^{k}(\partial T)$ had not been surjective (but nevertheless well defined) we would have gotten:

$$
\operatorname{dim} A^{k}(\mathcal{T}) \leq \sum_{T \in \mathcal{T}} \operatorname{dim} A_{0}^{k}(T) .
$$

Notice that we have not constructed any explicit isomorphism between the spaces $A^{k}(\mathcal{T})$ and $\bigoplus_{T \in \mathcal{T}} A_{0}^{k}(T)$, even though we proved that such an isomorphism exists.

Example 2.5 Given a cellular complex $\mathcal{T}$ one can fix a simplicial refinement $\mathcal{T}^{\prime}$ and let $A^{k}(T)$ denote the Whitney $k$-forms on $T$ with respect to $\mathcal{T}^{\prime}$. We proved that the spaces $A^{k}(T)$ have the right cohomology in Corollary 2.13. The restriction property is trivial. Thus we have a compatible finite element system. It is clear that $\Lambda^{k}\left(\mathcal{T}^{\prime}\right) \subseteq A^{k}(\mathcal{T})$. Comparing dimensions one finds that the spaces are equal.

Theorem 2.16 When the finite element system is compatible the De Rham map $\mu^{\bullet}: A^{\bullet}(\mathcal{T}) \rightarrow C^{\bullet}(\mathcal{T})$ induces isomorphisms in cohomology.

- Proof: We suppose that the theorem has been proved for cellular complexes of dimension up to $n$. We let $\mathcal{T}^{\prime}$ be a cellular complex of dimension at most $n+1$ for which the theorem is true, and adjoint an $(n+1)$ dimensional cell $T$ with boundary in $\mathcal{T}^{\prime}$. Let $\mathcal{T}$ denote the cellular complex $\mathcal{T}^{\prime} \cup\{T\}$.

We have short exact sequences:

$$
0 \rightarrow A^{k}(\mathcal{T}) \rightarrow A^{k}\left(\mathcal{T}^{\prime}\right) \times A^{k}(T) \rightarrow A^{k}(\partial T) \rightarrow 0,
$$

where the second arrow is:

$$
u \mapsto\left(\left.u\right|_{\mathcal{T}^{\prime}},\left.u\right|_{T}\right),
$$


and the third arrow is:

$$
\left.(u, v) \mapsto u\right|_{\partial T}-\left.v\right|_{\partial T}
$$

It provides a long exact sequence. We have similar short exact sequences for $C^{\bullet}$ and a corresponding long exact sequence. They are related by the De Rham map providing a commuting diagram:

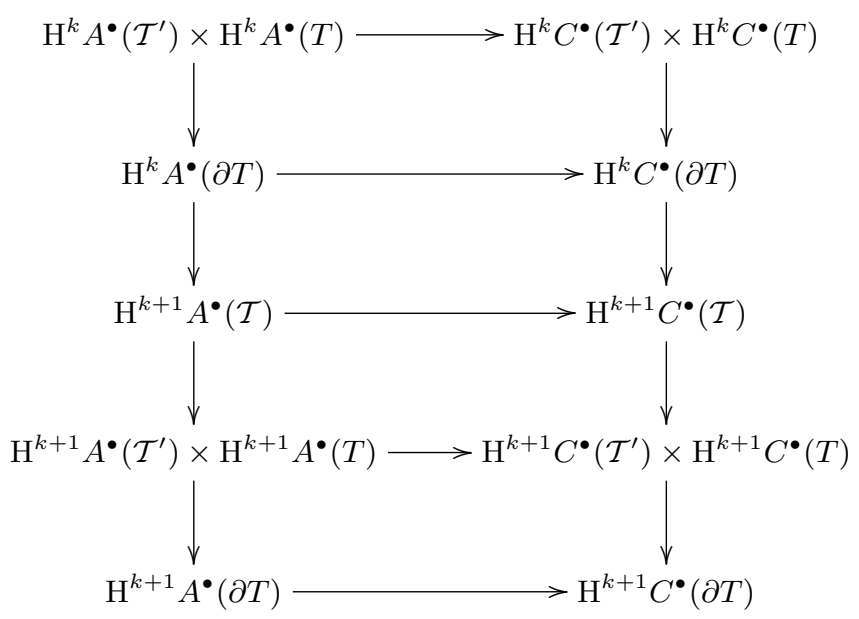

The first, second fourth, and fifth horizontal arrows are isomorphisms by the induction hypothesis (remark that $\partial T$ is $n$-dimensional), hence also the third.

Corollary 2.17 Let $\mathcal{T}$ be a cellular complex and $\mathcal{T}^{\prime}$ a simplicial refinement. Then the natural map $C^{\bullet}\left(\mathcal{T}^{\prime}\right) \rightarrow C^{\bullet}(\mathcal{T})$ induces isomorphisms in cohomology.

- Proof: We use the finite element system of Example 2.5. By Theorem 2.16 the natural morphism $\Lambda^{\bullet}\left(\mathcal{T}^{\prime}\right) \rightarrow C^{\bullet}(\mathcal{T})$ induces isomorphisms in cohomology. But $\Lambda^{\bullet}\left(\mathcal{T}^{\prime}\right)$ is naturally isomorphic to $C^{\bullet}\left(\mathcal{T}^{\prime}\right)$.

Corollary 2.18 Let $\mathcal{T}$ be a cellular complex and $\mathcal{T}^{\prime}$ a cellular refinement. Then the natural map $C^{\bullet}\left(\mathcal{T}^{\prime}\right) \rightarrow C^{\bullet}(\mathcal{T})$ induces isomorphisms in cohomology.

- Proof: Let $\mathcal{T}^{\prime \prime}$ be a simplicial refinement of $\mathcal{T}^{\prime}$. Proposition 2.8 provides a commuting diagram of complexes:

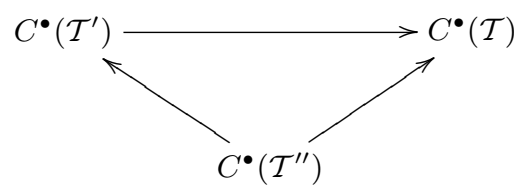

The two diagonal arrows induce isomorphisms in cohomology as noted in the previous corollary. Hence the horizontal arrow also induces isomorphisms.

Corollary 2.19 Let $M$ be manifold and $\mathcal{T}$ a cellular complex. The De Rham map $\Omega^{\bullet}(M) \rightarrow C^{\bullet}(\mathcal{T})$ induces isomorphisms in cohomology. 
- Proof: Let $\mathcal{T}^{\prime}$ be a simplicial refinement of $\mathcal{T}$. We have a commuting diagram of complexes:

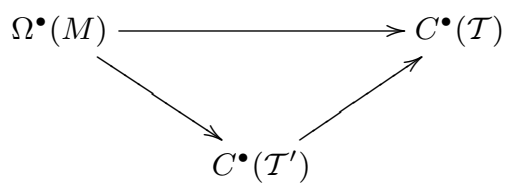

The two diagonal arrows induce isomorphisms in cohomology by Theorem 2.14 and Corollary 2.17. Hence the horizontal arrow also induces isomorphisms.

Harmonic extensions We now introduce a notion of harmonic extension. It has at least two applications. It yields an isomorphism $\bigoplus_{T \in \mathcal{T}} A_{0}^{k}(T) \rightarrow$ $A^{k}(\mathcal{T})$ and also a subcomplex of $A^{\bullet}(\mathcal{T})$ such that the restriction of the De Rham map to this subcomplex determines isomorphisms to $C^{\bullet}(\mathcal{T})$ (not just in cohomology). This subcomplex plays the role of lowest order Whitney forms on general cellular complexes.

Let $T$ be a cell of dimension $p$. Then $C_{0}^{k}(T)=0$ for $k<p$ and $C_{0}^{p}(T) \simeq \mathbb{R}$.

Proposition 2.20 The following sequence is exact:

$$
0 \rightarrow A_{0}^{0}(T) \rightarrow A_{0}^{1}(T) \rightarrow \cdots \rightarrow A_{0}^{\operatorname{dim}(T)}(T) \rightarrow \mathbb{R} \rightarrow 0 .
$$

- Proof: We have a diagram of short exact sequences:

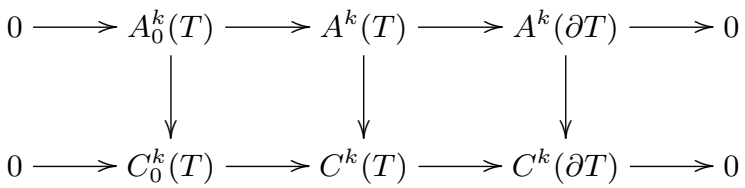

The two last vertical arrows induce isomorphisms in cohomology by Theorem 2.16, hence, by applying the five lemma to the long exact sequences, so does the first. But the cohomology of $C_{0}^{\bullet}(T)$ is trivial to compute.

From now on we suppose that a scalar product $a$ is given on each $A^{k}(T)$.

Lemma 2.7 For each $k, T \in \mathcal{T}^{k}$ and $\alpha \in \mathbb{R}$ there is a unique element $u$ of $A^{\operatorname{dim} T}(T)$ such that:

$$
\int_{T} u=\alpha \text { and } \forall v \in A_{0}^{\operatorname{dim} T-1}(T) \quad a(u, \mathrm{~d} v)=0 .
$$

- Proof: The orthogonal of im d : $A_{0}^{\operatorname{dim} T-1}(T) \rightarrow A_{0}^{\operatorname{dim} T}(T)$ with respect to $a$ is one-dimensional and contains an element with nonzero integral.

With the above notations, the element with integral 1 will be denoted $\omega_{T}$.

Lemma 2.8 Pick a $k$. For each $T \in \mathcal{T}$ such that $\operatorname{dim} T>k$ if $u \in$ $A^{k}(\partial T)$ there is a unique extension $u \in A^{k}(T)$ such that:

$$
\forall v \in A_{0}^{k}(T) \quad a(\mathrm{~d} u, \mathrm{~d} v)=0 \text { and } \forall v \in A_{0}^{k-1}(T) \quad a(u, \mathrm{~d} v)=0 .
$$


- Proof: Put $K=$ imd $: A_{0}^{k-1}(T) \rightarrow A_{0}^{k}(T)$. Then on $K, a$ is of course a scalar product but more importantly on its orthogonal $K^{\perp}$ in $A_{0}^{k}(T)$ with respect to $a, a(\mathrm{~d} \cdot, \mathrm{d} \cdot)$ is also a scalar product since $\operatorname{dim} T>k$ and therefore $K=\operatorname{kerd}: A_{0}^{k}(T) \rightarrow A_{0}^{k+1}(T)$. Pick now $u_{0} \in A^{k}(T)$, an arbitrary extension of $u$. If $u_{1}$ and $u_{2}$ are in $K^{\perp}$ and $K$ respectively then $u=u_{0}+u_{1}+u_{2}$ solves our problem if and only if:

$\forall v \in K^{\perp} \quad a\left(\mathrm{~d} u_{1}, \mathrm{~d} v\right)=-a\left(\mathrm{~d} u_{0}, \mathrm{~d} v\right)$ and $\forall v \in K \quad a\left(u_{2}, v\right)=-a\left(u_{0}, v\right)$.

This gives existence and uniqueness.

A differential form $u \in A^{k}(T)$ such that:

$$
\forall v \in A_{0}^{k}(T) \quad a(\mathrm{~d} u, \mathrm{~d} v)=0 \text { and } \forall v \in A_{0}^{k-1}(T) \quad a(u, \mathrm{~d} v)=0 .
$$

will be called harmonic. We say that a differential form $u \in A^{k}(\mathcal{T})$ is locally harmonic if for each $T \in \mathcal{T},\left.u\right|_{T}$ is harmonic.

The construction we propose is the following: Fix a $k$ and a $T \in \mathcal{T}^{k}$. We will construct a $\lambda_{T} \in \Omega^{k}$ attached to $T$.

- First (using Lemma 2.7) put $\left.\lambda_{T}\right|_{T}=\omega_{T}$ and for each $T^{\prime} \in \mathcal{T}^{k}$ such that $T^{\prime} \neq T$ we put $\left.\lambda_{T}\right|_{T^{\prime}}=0$. Of course $\lambda_{T}$ is set to zero also on cells of dimension $i<k$.

- Second (using Lemma 2.8), supposing $\lambda_{T}$ has been defined on all cells of dimension up to some $i \geq k$ we define $\left.\lambda_{T}\right|_{T^{\prime}}$ on a cell $T^{\prime} \in \mathcal{T}^{i+1}$ to be the unique element $u \in A^{k}\left(T^{\prime}\right)$ with $\left.u\right|_{\partial T^{\prime}}$ given by $\left.\lambda_{T}\right|_{\partial T^{\prime}}$ and such that $u$ is harmonic.

We now put:

$$
\Lambda^{k}(\mathcal{T})=\operatorname{span}\left\{\lambda_{T}: T \in \mathcal{T}^{k}\right\}
$$

We have:

Proposition 2.21 $\Lambda^{k}(\mathcal{T})$ is the set of locally harmonic elements of $A^{k}(\mathcal{T})$. The family $\left(\lambda_{T}\right)$ indexed by $T \in \mathcal{T}^{k}$ is a basis of $\Lambda^{k}(\mathcal{T})$, and the induced map $\mu^{k}: \Lambda^{k}(\mathcal{T}) \rightarrow C^{k}(\mathcal{T})$ is an isomorphism. The exterior derivative induces maps $\mathrm{d}: \Lambda^{k}(\mathcal{T}) \rightarrow \Lambda^{k+1}(\mathcal{T})$.

- Proof: Since, for any $T$ of dimension $k, \omega_{T}$ is harmonic (on $T$ ), any element of $\Lambda^{k}(\mathcal{T})$ is locally harmonic. Since, for any $T$ of dimension $k$ any element of $A^{k}(T)$ which is harmonic is proportional to $\omega_{T}$, locally harmonic forms are in $\Lambda^{k}(\mathcal{T})$.

We notice that for $T, T^{\prime} \in \mathcal{T}^{k}$ we have $\mu_{T^{\prime}} \lambda_{T}=\delta_{T T^{\prime}}$ where the last symbol is the Kronecker delta, hence $\left(\lambda_{T}\right)$ is linearly independent. It is therefore a basis of $\Lambda^{k}(\mathcal{T})$. The De Rham map sends this basis to the canonical basis of $C^{k}(\mathcal{T})$.

We now prove that $\mathrm{d} \Lambda^{k}(\mathcal{T}) \subseteq \Lambda^{k+1}$. But it is trivial to check that if $u$ is locally harmonic, so is $\mathrm{d} u$, so the result follows.

Remark 2.10 The maps $\mu^{k}$ can be used to define interpolation operators $I^{k}: \Omega^{k} \rightarrow \Lambda^{k}(\mathcal{T})$ by requiring, for any $u \in \Omega^{k}$ that $\mu^{k} I^{k} u=\mu^{k} u$. They commute with the exterior derivative.

Proposition 2.22 We have:

$$
\sum_{i \in \mathcal{T}^{0}} \lambda_{i}=1
$$


- Proof: Define $u \in \Lambda^{0}$ by:

$$
u=\sum_{i \in \mathcal{T}^{0}} \lambda_{i}
$$

We prove that $u=1$ by induction on the dimension of the cells. For a 0 -dimensional cell $T$ it is of course true that $\left.u\right|_{T}=1$. Suppose now it has been proved that $\left.u\right|_{T}=1$ for all cells $T$ of dimension $\leq k$ and consider a $(k+1)$-dimensional cell $T$. The constant function on $T$ equal to 1 is an element of $A^{0}(T)$ whose boundary values are 1 and whose exterior derivative is 0 . By the uniqueness proved Lemma 2.8, we therefore have $\left.u\right|_{T}=1$.

It is therefore tempting to believe that the family $\left(\lambda_{i}\right)_{i \in \mathcal{T}^{0}}$ is a partition of unity. However, in general, the functions $\lambda_{i}$ might take negative values. For instance one can use finite element functions on a refinement of $\mathcal{T}$ to construct the spaces $A^{0}(T)$ and use $\mathrm{L}^{2}$ products for $a$. Then the constructed functions are so-called discrete harmonic on each cell, but it is known that the (refined) mesh needs to satisfy additional requirements for discrete maximum principles to hold.

When $M$ is a domain in a vectorspace $V$ it makes sense to speak about constant forms on $M$. One might then ask that $\Lambda^{k}(\mathcal{T})$ contains all compatible differential forms which are constant on each top-dimensional cell. However in general this is too much to ask. Indeed the pullbacks to a $k$-dimensional cell $T$ of elements of $\Lambda^{k}(\mathcal{T})$ form a one-dimensional space (generated by $\omega_{T}$ ), whereas the pullbacks to $T$ of constant $k$-forms on $V$ might form a space of higher dimension. Notice that this is not a problem of choice of auxiliary spaces and scalar products but comes from our requirement that for $k$-forms we have one degree of freedom per $k$-dimensional cell, whereas more would be required to represent the pullbacks of constant $k$-forms.

We say that a $k$-dimensional cell is flat if it is included in a $k$-dimensional affine space. The next proposition shows that when the cells are all flat the previous problem does not occur.

Proposition 2.23 Suppose we have a scalar product on $V$ and that we use for scalar product a on a cell the $\mathrm{L}^{2}$ product on forms associated with the induced Riemannian metric. Suppose that each cell is flat and that for each $k$ and $T, A^{k}(T)$ contains the pullbacks of constant forms on $V$. Then $\Lambda^{k}(\mathcal{T})$ contains all compatible differential forms on $M$ which are constant on each top-dimensional cell.

- Proof: Indeed for any constant differential form on $V$, the exterior derivative of its pullback to any cell is 0 (by commutation of the exterior derivative with pullbacks). Moreover if $u$ is the pullback to a cell $T$ of a constant differential form $\mathrm{d}^{\star} u=0$ by the flatness of $T$.

Therefore compatible differential forms on $M$ which are constant on each top-dimensional cell are piecewise harmonic.

When the hypothesis of Proposition 2.23 is satisfied, the canonical interpolation operator defined by Remark 2.10 can be used to obtain the basic error estimates. The only problem is that the canonical interpolation operator is not defined on rough forms, say with mere $\mathrm{L}^{2}$ regularity, but this can be corrected for using the techniques of [29] or [56].

One application of this construction is the following. Suppose a simplicial mesh $\mathcal{T}$ is given for an $n$-dimensional oriented manifold. One equips it with the Whitney forms $\Lambda^{\bullet}(\mathcal{T})$ and wishes to construct finite element 
spaces of $k$-forms $\Gamma^{k}$ forming a complex such that the bilinear forms:

$$
\left\{\begin{aligned}
\Lambda^{k}(\mathcal{T}) \times \Gamma^{n-k} & \rightarrow \mathbb{R} \\
(u, v) & \mapsto \int u \wedge v
\end{aligned}\right.
$$

are non-degenerate. One can perform the barycentric refinement of $\mathcal{T}$. It yields a simplicial refinement $\mathcal{T}^{\prime}$ of $\mathcal{T}$ whose vertices are all the barycenters of all cells of $\mathcal{T}$. Then one can reassemble the elements of $\mathcal{T}^{\prime}$ into a new cellular complex $\mathcal{U}$ called the dual complex of $\mathcal{T}$, whose $k$-cells are transverse to the $(n-k)$-cells of $\mathcal{T}$. The locally harmonic forms on $\mathcal{U}$ constructed from Whitney forms on $\mathcal{T}^{\prime}$ provide a good candidate for $\Gamma^{\bullet}$. We proved that the bilinear forms (37) are non-degenerate in dimension $n=2$ in [18], but the case of general dimension remains open. Such dual Whitney forms are useful for preconditioning integral operators discretized on (primal) Whitney forms [2].

Remark that on simplicial complexes we now have two definitions of $\lambda_{T}$, as (lowest order) Whitney forms and as locally harmonic ones constructed from some finite element system. We proceed to show that Whitney forms are locally harmonic with respect to any piecewise constant metric, so that in fact there is hardly any ambiguity. Denote by $\lambda_{i}$ the barycentric coordinate map associated with any vertex $i \in \mathcal{T}^{0}$. Recall that for $k \geq 1$, if $T \in \mathcal{T}^{k}$ and we label (a bit abusively) its vertexes $0,1, \cdots, k$ in a manner consistent with the orientation of $T$ the Whitneyform associated with $T$ is:

$$
\lambda_{T}=\sum_{\sigma} \epsilon(\sigma) \lambda_{\sigma(0)} \mathrm{d} \lambda_{\sigma(1)} \wedge \cdots \wedge \mathrm{d} \lambda_{\sigma(k)},
$$

where the sum extends over all permutations of $\{0,1, \cdots, k\}$, and $\epsilon$ is the signature morphism (see [61]). All we have to check is that, on any cell $T^{\prime}, \mathrm{d}^{\star} \mathrm{d} \lambda_{T}=0$ and $\mathrm{d}^{\star} \lambda_{T}=0$. But we have:

$$
\mathrm{d} \lambda_{T}=(k+1) ! \mathrm{d} \lambda_{0} \wedge \mathrm{d} \lambda_{1} \wedge \cdots \wedge \mathrm{d} \lambda_{k},
$$

which is constant on any simplex, so the first condition is true. Concerning the second condition, we remark that for $k=0$ it is trivial. For $k \geq 1$ we use the Hodge star operator. When $g$ is a metric on some $n$-dimensional oriented space $E$ and $\omega$ is the corresponding volume form, the Hodge star maps $k$-forms to $(n-k)$-forms and is characterized by the property, for all $k$-forms $u$ and $v$ :

$$
u \wedge \star v=g(u, v) \omega .
$$

One then checks that $\mathrm{d}^{\star}= \pm \star \mathrm{d} \star$. We have, since $g$ is constant on $T^{\prime}$ :

$$
\mathrm{d} \star \lambda_{T}=\sum_{\sigma} \epsilon(\sigma) \mathrm{d} \lambda_{\sigma(0)} \wedge \star\left(\mathrm{d} \lambda_{\sigma(1)} \wedge \cdots \wedge \mathrm{d} \lambda_{\sigma(k)}\right),
$$

This is a certain constant $(n-k+1)$-form on $T^{\prime}$ and we wish to show that it is zero. This is the object of the following lemma.

Lemma 2.9 Let $V$ be an oriented Euclidean space, $k \geq 1$ an integer and $v_{0}, v_{1}, \cdots, v_{k}$ a family of linear forms on $V$. Then:

$$
\sum_{\sigma} \epsilon(\sigma) v_{\sigma(0)} \wedge \star\left(v_{\sigma(1)} \wedge \cdots \wedge v_{\sigma(k)}\right)=0
$$

- Proof: Let $(\cdot \mid \cdot)$ denote the scalar product and $\omega$ be the volume form. If $k=1$ we have:

$$
v_{0} \wedge \star v_{1}-v_{1} \wedge \star v_{0}=\left(v_{0} \mid v_{1}\right) \omega-\left(v_{1} \mid v_{0}\right) \omega=0,
$$


which is the identity we wanted to prove.

Suppose now $k \geq 2$. If $u \in \mathrm{L}_{a}^{k-1}(V)$ we have:

$u \wedge \sum_{\sigma} \epsilon(\sigma) v_{\sigma(0)} \wedge \star\left(v_{\sigma(1)} \wedge \cdots \wedge v_{\sigma(k)}\right)=\sum_{\sigma} \epsilon(\sigma)\left(u \wedge v_{\sigma(0)} \mid v_{\sigma(1)} \wedge \cdots \wedge v_{\sigma(k)}\right) \omega$.

Denote by $S$ the scalar coefficient in front of $\omega$. We have:

$$
S=\sum_{\sigma} \epsilon(\sigma)\left(u \wedge v_{\sigma(0)} \mid v_{\sigma(1)} \otimes \cdots \otimes v_{\sigma(k)}\right) .
$$

Now if $u$ is decomposable, i.e. of the form $u=u_{1} \wedge \cdots \wedge u_{k-1}$, with $u_{i} \in V^{\star}$, we have:

$$
\begin{gathered}
u \wedge v_{\sigma(0)}=(1 / k !) \sum_{i=1}^{k} \sum_{\tau}(-1)^{i} \epsilon(\tau) u_{\tau(1)} \otimes \cdots \otimes u_{\tau(i-1)} \otimes \\
v_{\sigma(0)} \otimes u_{\tau(i)} \otimes \cdots \otimes u_{\tau(k-1)},
\end{gathered}
$$

where the second sum is over all permutations $\tau$ of the set $\{1, \cdots, k-1\}$. Inserting this into the expression for $S$ we get:

$$
\begin{aligned}
S=(1 / k !) \sum_{i, \tau, \sigma}(-1)^{i} \epsilon(\tau) \epsilon(\sigma)\left(u_{\tau(1)} \otimes \cdots \otimes u_{\tau(i-1)} \otimes\right. \\
\left.v_{\sigma(0)} \otimes u_{\tau(i)} \otimes \cdots \otimes u_{\tau(k-1)} \mid v_{\sigma(1)} \otimes \cdots \otimes v_{\sigma(k)}\right)
\end{aligned}
$$

We now fix $i$ and $\tau$ and remark that an uneven permutation of $\{0, \cdots, k\}$ can be uniquely written $\sigma \pi$ where $\pi$ is the permutation exchanging 0 and $i$. Thus in the above sum we can sum over all even permutations $\tau$, two terms - one like above and one where $v_{\sigma(0)}$ and $v_{\sigma(i)}$ are switched places:

$$
\begin{aligned}
\left(u_{\tau(1)} \otimes \cdots \otimes u_{\tau(i-1)} \otimes v_{\sigma(0)} \otimes u_{\tau(i)} \otimes \cdots \otimes u_{\tau(k-1)} \mid v_{\sigma(1)} \otimes \cdots \otimes v_{\sigma(k)}\right) \\
-\left(u_{\tau(1)} \otimes \cdots \otimes u_{\tau(i-1)} \otimes v_{\sigma(i)} \otimes u_{\tau(i)} \otimes \cdots \otimes u_{\tau(k-1)} \mid\right. \\
\left.v_{\sigma(1)} \otimes \cdots \otimes v_{\sigma(i-1)} \otimes v_{\sigma(0)} \otimes v_{\sigma(i+1)} \otimes \cdots \otimes v_{\sigma(k)}\right)
\end{aligned}
$$

Here the sign reflects that $\sigma \pi$ is an uneven permutation. The above sum is 0 by symmetry of the scalar product. Since this holds for all decomposable $u$ it is true for all $u \in \mathrm{L}_{a}^{k-1}(V)$. This proves the lemma.

Finally notice that an element of $A_{0}^{k}(T)$ can be extended by 0 to an element of $A^{l}\left(\mathcal{T}^{(l)}\right)$ where $l=\operatorname{dim} T$ and $\mathcal{T}^{(l)}$ is the $l$-skeleton of $\mathcal{T}$. It can thereafter be extended recursively to cells of higher and higher dimension in a unique way requiring harmonicity at each stage. This yields linear maps $E: A_{0}^{k}(T) \rightarrow A^{k}(\mathcal{T})$ which can be thought of as a global map:

$$
E: \bigoplus_{T} A_{0}^{\bullet}(T) \rightarrow A^{\bullet}(\mathcal{T})
$$

respecting the degree of differential forms. Notice that for $k<\operatorname{dim} T$ if $u \in A_{0}^{k}(T)$ then $\mathrm{d} E u=E \mathrm{~d} u$. When $k=\operatorname{dim} T$, if $u \in A_{0}^{k}(T)$ then its harmonic extension will have non-zero exterior derivative if $u$ has non-zero integral.

Proposition 2.24 The map $E: \oplus_{T} A_{0}^{\bullet}(T) \rightarrow A^{\bullet}(\mathcal{T})$ is an isomorphism.

- Proof: The extension operator $E: A_{0}^{k}(T) \rightarrow A^{k}(\mathcal{T})$ has the property that for any $u \in A_{0}^{k}(T)$ and any $T^{\prime}$ such that $T \nsubseteq T^{\prime},\left.(E u)\right|_{T^{\prime}}=0$.

If now $u \in \bigoplus_{T} A_{0}^{\bullet}(T)$ is such that $E u=0$ one deduces that $\left.u\right|_{T}=0$ starting from cells $T$ of dimension $k$ and inductively increasing dimension. 
Injectivity yields bijectivity by equality of dimension.

Another extension operator defined for smooth differential forms was used in the preprint of [22] to prove that compatible piecewise smooth differential forms can be extended from the boundary of a simplex to its interior, showing that the spaces of "all" compatible piecewise smooth differential forms constitute a finite element system. An axiomatic treatment of extension operators is given in [6]. It covers two notable other cases: extension using the degrees of freedom of finite elements and extension using Bernstein polynomials.

Such extension operators reduce the problem of constructing a basis for the global space $A^{k}(\mathcal{T})$ to that of constructing bases for the local spaces $A_{0}^{k}(T)$. See for instance [57].

Notice furthermore that the construction of locally harmonic forms can be applied recursively. Given a (fine) cellular complex $\mathcal{T}_{0}$ for which one has a compatible finite element system (e.g. a simplicial complex), one can assemble the cells into a coarser complex $\mathcal{T}_{1}$, whose cells can again be assembled into a coarser complex $\mathcal{T}_{2}$ etc. At each stage one can construct the space of locally harmonic forms on $\mathcal{T}_{k+1}$ from the finite element system provided by $\mathcal{T}_{k}$. This provides nested spaces:

$$
\Lambda^{\bullet}\left(\mathcal{T}_{k+1}\right) \subseteq \Lambda^{\bullet}\left(\mathcal{T}_{k}\right) \subseteq \cdots \subseteq \Lambda^{\bullet}\left(\mathcal{T}_{0}\right)
$$

which can be used for instance for preconditioning. See [51].

Constructions with finite element systems We now show that the notion of finite element system we introduced behaves naturally with respect to tensor products, that degrees of freedom can be readily defined and that it can also be used to describe $h p$ - finite elements.

Suppose we have two manifolds $M$ and $N$, equipped with cellular complexes $\mathcal{T}$ and $\mathcal{U}$, and auxiliary spaces $A^{k}(T)$ for $T \in \mathcal{T}$ and $B^{k}(U)$ for $U \in \mathcal{U}$ subject to the above conditions on cohomology and restrictions.

Let $\mathcal{T} \times \mathcal{U}$ denote the product cellular complex, whose cells are of the form $T \times U$ for $T \in \mathcal{T}$ and $U \in \mathcal{U}$. We equip $\mathcal{T} \times \mathcal{U}$ with auxiliary spaces:

$$
Z^{\bullet}(T \times U)=A^{\bullet}(T) \otimes B^{\bullet}(U) .
$$

Explicitly we put:

$$
Z^{k}(T \times U)=\bigoplus_{l} A^{l}(T) \otimes B^{k-l}(U)
$$

where the tensorproduct is that of differential forms.

Proposition $2.25 Z^{\bullet}$ has the right cohomological properties on any product cell.

- Proof: This follows from Theorem 2.5.

We now prove in several steps that the restrictions to boundaries in $Z^{\bullet}$ is surjective, so that $Z^{\bullet}$ fulfils the requirements to be a finite element system.

Lemma 2.10 We have:

$$
Z_{0}^{\bullet}(T \times U)=A_{0}^{\bullet}(T) \otimes B_{0}^{\bullet}(U)
$$


- Proof: Let $\left(u_{i}\right)_{0<i<m}$ denote a basis of $A_{0}^{\bullet}(T)$ and extend it to a basis $\left(u_{i}\right)_{0 \leq i<m^{\prime}}$ of $A^{\bullet}(T)$. Similarly let $\left(v_{j}\right)_{0 \leq j<n}$ denote a basis of $B_{0}^{\bullet}(U)$ and extend it to a basis $\left(v_{j}\right)_{0 \leq j<n^{\prime}}$ of $B^{\bullet}(U)$.

Now pick $z \in Z^{\bullet}(T \times U)$ and write it in the form:

$$
z=\sum_{\substack{0 \leq i<m^{\prime} \\ 0 \leq j<n^{\prime}}} \lambda_{i j} u_{i} \otimes v_{j},
$$

and suppose that $z$ restricted to the boundary of $T \times U$ is 0 .

Restricting $z$ to $\partial T \times \partial U$ we get:

$$
\left.\left.\sum_{\substack{m \leq i<m^{\prime} \\ n \leq j<n^{\prime}}} \lambda_{i j} u_{i}\right|_{\partial T} \otimes v_{j}\right|_{\partial U}=0 .
$$

But we remark that $\left(\left.u_{i}\right|_{\partial T}\right)_{m \leq i<m^{\prime}}$ is linearly independent, as well as $\left(\left.v_{j}\right|_{\partial U}\right)_{n \leq j<n^{\prime}}$. It follows that $\lambda_{i j}=0$ for $m \leq i<m^{\prime}$ and $n \leq j<n^{\prime}$.

Next one restricts $z$ to $\partial T \times U$ and $T \times \partial U$ (both included in the boundary of $T \times U)$ and get by the same remark that $\lambda_{i j}=0$ for $m \leq i<$ $m^{\prime}$ and $0 \leq j<n$ as well as for $0 \leq i<m$ and $n \leq j<n^{\prime}$.

Thus we get:

$$
z=\sum_{\substack{0 \leq i<m \\ 0 \leq j<n}} \lambda_{i j} u_{i} \otimes v_{j}
$$

and we are done.

Proposition 2.26 We have:

$$
Z^{\bullet}(\mathcal{T} \times \mathcal{U})=A^{\bullet}(\mathcal{T}) \otimes B^{\bullet}(\mathcal{U})
$$

- Proof: It is clear that:

$$
A^{\bullet}(\mathcal{T}) \otimes B^{\bullet}(\mathcal{U}) \subseteq Z^{\bullet}(\mathcal{T} \times \mathcal{U})
$$

We prove that the spaces have the same dimension. By Remark 2.9 we have:

$$
\begin{aligned}
\operatorname{dim} Z^{\bullet}(\mathcal{T} \times \mathcal{U}) & \leq \sum_{T U} \operatorname{dim} Z_{0}^{\bullet}(T \times U), \\
\leq & \sum_{T U} \operatorname{dim}\left(A_{0}^{\bullet}(T) \otimes B_{0}^{\bullet}(U)\right), \\
\leq & \sum_{T U} \operatorname{dim} A_{0}^{\bullet}(T) \operatorname{dim} B_{0}^{\bullet}(U), \\
\leq & \sum_{T} \operatorname{dim} A_{0}^{\bullet}(T) \sum_{U} \operatorname{dim} B_{0}^{\bullet}(U), \\
\leq & \operatorname{dim} A^{\bullet}(\mathcal{T}) \operatorname{dim} B^{\bullet}(\mathcal{U}) .
\end{aligned}
$$

Thus we get:

$$
\operatorname{dim} Z^{\bullet}(\mathcal{T} \times \mathcal{U}) \leq \operatorname{dim}\left(A^{\bullet}(\mathcal{T}) \otimes B^{\bullet}(\mathcal{U})\right)
$$

This completes the proof.

Proposition 2.27 For any cell $T \in \mathcal{T}, U \in \mathcal{U}$, the restriction $Z^{\bullet}(T \times$ $U) \rightarrow Z^{\bullet}(\partial(T \times U))$ is onto.

- Proof: Pick $z \in Z^{\bullet}(\partial(T \times U))$.

We have:

$$
\left.z\right|_{\partial T \times \partial U} \in Z^{\bullet}(\partial T \times \partial U)=A^{\bullet}(\partial T) \otimes B^{\bullet}(\partial U) .
$$


Such a form may therefore be extended from $\partial T \times \partial U$ to $T \times U$ (by extending each component in the sum of tensorproducts it can be represented by on $\partial T \times \partial U)$.

We are therefore left with the problem of extending a $z \in Z^{\bullet}(\partial(T \times U))$ such that $\left.z\right|_{\partial T \times \partial U}=0$.

We remark that we have a short exact sequence:

$$
0 \rightarrow A_{0}^{\bullet}(T) \rightarrow A^{\bullet}(T) \rightarrow A^{\bullet}(\partial T) \rightarrow 0 .
$$

Taking the tensor products with $B^{\bullet}(\partial U)$ and using Proposition 2.26, we get a short exact sequence:

$$
0 \rightarrow A_{0}^{\bullet}(T) \otimes B^{\bullet}(\partial U) \rightarrow Z^{\bullet}(T \times \partial U) \rightarrow Z^{\bullet}(\partial T \times \partial U) \rightarrow 0 .
$$

Similarly we have a short exact sequence:

$$
0 \rightarrow A^{\bullet}(\partial T) \otimes B_{0}^{\bullet}(U) \rightarrow Z^{\bullet}(\partial T \times U) \rightarrow Z^{\bullet}(\partial T \times \partial U) \rightarrow 0 .
$$

Pick now $z \in Z^{\bullet}(\partial(T \times U))$ such that $\left.z\right|_{\partial T \times \partial U}=0$.

We have:

$$
\left.z\right|_{T \times \partial U} \in A_{0}^{\bullet}(T) \otimes B^{\bullet}(\partial U) .
$$

Thus we can find $z^{\prime} \in A_{0}^{\bullet}(T) \otimes B^{\bullet}(U)$ such that $\left.z^{\prime}\right|_{T \times \partial U}=\left.z\right|_{T \times \partial U}$.

Similarly we have:

$$
\left.z\right|_{\partial T \times U} \in A^{\bullet}(\partial T) \otimes B_{0}^{\bullet}(U) .
$$

Hence we can find $z^{\prime \prime} \in A^{\bullet}(T) \otimes B_{0}^{\bullet}(U)$ such that $\left.z^{\prime \prime}\right|_{\partial T \times U}=\left.z\right|_{\partial T \times U}$.

We then have:

$$
\left.\left(z^{\prime}+z^{\prime \prime}\right)\right|_{T \times \partial U}=\left.z^{\prime}\right|_{T \times \partial U}=\left.z\right|_{T \times \partial U},
$$

and similarly :

$$
\left.\left(z^{\prime}+z^{\prime \prime}\right)\right|_{\partial T \times U}=\left.z^{\prime \prime}\right|_{\partial T \times U}=\left.z\right|_{\partial T \times U},
$$

Since:

$$
(T \times \partial U) \cup(\partial T \times U)=\partial(T \times U),
$$

the form $z^{\prime}+z^{\prime \prime}$ is a suitable extension of $z$.

Thus when $A$ and $B$ are compatible finite element systems, $Z$ also. There are now two ways of constructing a complex of spaces isomorphic to $C^{\bullet}(\mathcal{T} \times \mathcal{U})$ : either construct first the spaces of locally harmonic forms on $M$ and $N$ from $A$ and $B$ and then take the tensor product, or construct the locally harmonic forms on $M \times N$ from $Z$. If the scalar product chosen for each product cell $T \times U$ is the product of the scalar product of $T$ and the scalar product of $U$, then these two constructions yield the same spaces. It suffices to check that the tensor product of two locally harmonic forms is locally harmonic.

So far the only example of a finite element system we have considered on a simplicial complex is the one provided by Whitney forms. One can also construct high order Whitney forms by taking products with polynomials [49] [38] [5]. Specifically, for a simplex $T$ and integer $p \geq 1$ let $A^{k, p}(T)$ denote the space spanned by $k$-forms of the form $u \lambda_{T^{\prime}}$ where $u$ is a polynomial of degree at most $p-1, T^{\prime}$ is a $k$-face of $T$ and $\lambda_{T^{\prime}}$ the associated Whitney form. As we noted in the preprint of [22] the wedge product provides a map $A^{k, p}(T) \times A^{k^{\prime}, p^{\prime}}(T) \rightarrow A^{k+k^{\prime}, p+p^{\prime}}(T)$ (one says that the wedge product respects the filtration) which is the reason for letting the lowest order Whitney forms be indexed by $p=1$ rather than 
$p=0$ which was usual before. We also denote by $B^{k, p}(T)$ the space of polynomial differential $k$-forms of degree at most $p$.

Suppose a simplicial complex is given as well as a polynomial degree $p$. Each simplex is supposed to be oriented. That the system $A^{\bullet, p}(\cdot)$ for given $p$ constitutes a compatible finite element system follows immediately from results proved for instance in [5]. Exactness is a theorem whereas the extension property follows from the existence of degrees of freedom. We will also use that the bilinear forms:

$$
\left\{\begin{aligned}
A_{0}^{k, p}(T) \times B^{n-k, p+k-n-1}(T) & \rightarrow \mathbb{R} \\
(u, v) & \mapsto \int u \wedge v
\end{aligned}\right.
$$

are non-degenerate.

Consider $p$ to be fixed and denote by $A^{k}(T)=A^{k, p}(T)$ and $B^{l}(T)=$ $B^{l, p-l-1}(T)$. Consider the map $\Phi^{k}$ which to a compatible differential form $u$ associates with each $T \in \mathcal{T}$ the linear form $\left.v \mapsto \int_{T} u\right|_{T} \wedge v$ on $B^{\operatorname{dim} T-k}(T)$.

Proposition 2.28 $\Phi^{k}$ induces an isomorphism $A^{k}(\mathcal{T}) \rightarrow \bigoplus_{T \in \mathcal{T}} B^{\operatorname{dim} T-k}(T)^{\star}$

- Proof: If $\Phi^{k} u=0$ then $u$ is zero on $k$-dimensional cells, therefore also on $(k+1)$-dimensional cells, etc. using the above non-degeneracy at each level. This gives injectivity. Moreover we have equality of dimension.

The map $\Phi^{k}$ is said to associate with $u$ its degrees of freedom. One also says that $\Phi^{k}$ is unisolvent on $A^{k}(\mathcal{T})$.

To any compatible $k$-form $u$ we can associate the unique $v \in A^{k}(\mathcal{T})$ such that $\Phi^{k} v=\Phi^{k} u$. We define an interpolation operator $I^{k}$ by $I^{k} u=v$.

Proposition 2.29 Interpolation thus defined commutes with the exterior derivative.

- Proof: $\quad$ Let $u$ be a compatible $k$-form. Let $T$ be an $n$-dimensional simplex and $v \in B^{n-k-1}(T)$ :

$$
\begin{aligned}
\int_{T} \mathrm{~d} I^{k} u \wedge v & =\int_{\partial T} I^{k} u \wedge v \pm \int_{T} I^{k} u \wedge \mathrm{d} v \\
& =\int_{\partial T} u \wedge v \pm \int_{T} u \wedge \mathrm{d} v \\
& =\int_{T} \mathrm{~d} u \wedge v \\
& =\int_{T} I^{k+1} \mathrm{~d} u \wedge v
\end{aligned}
$$

The important properties are that $\mathrm{d} B^{n-k-1}(T) \subseteq B^{n-k}(T)$ (notice that polynomial degree decreases), and that $B^{n-k-1}$ is stable upon taking traces.

We see that the construction of an interpolator carries over whenever we have a cellular complex equipped with a compatible finite element system $A^{k}(T)$ together with spaces $B^{l}(T)$ forming a finite element system such that:

- The form $\int \cdot \wedge$. is non degenerate on $A_{0}^{k}(T) \times B^{\operatorname{dim} T-k}(T)$.

Thus, in a mirror system, instead on focusing on cohomology and extension, we focus on duality. We call such a system of spaces a mirror system. We also say that $B^{\operatorname{dim} T-k}(T)$ is a mirror for $A_{0}^{k}(T)$.

Supposing we now have two manifolds $M$ and $M^{\prime}$ and corresponding cellular complexes $\mathcal{T}$ and $\mathcal{T}^{\prime}$, suppose that for both we have a finite 
element system $A$ and a mirror system $B$. Let $A$ denote also the tensor product finite element system and $B$ the tensorproduct of the mirror systems. That is for each product cell $T \times T^{\prime}$ :

$$
B^{\bullet}\left(T \times T^{\prime}\right)=B^{\bullet}(T) \otimes B^{\bullet}\left(T^{\prime}\right)
$$

Proposition 2.30 The tensor product of two mirror systems is a mirror system.

- Proof: $\quad$ Let $\operatorname{dim} T=n$ and $\operatorname{dim} T^{\prime}=n^{\prime}$. We have:

$$
A_{0}^{k}\left(T \times T^{\prime}\right)=\bigoplus_{l} A_{0}^{l}(T) \otimes A_{0}^{k-l}\left(T^{\prime}\right) .
$$

On the other hand:

$$
B^{n+n^{\prime}-k}\left(T \times T^{\prime}\right)=\bigoplus_{l} B^{n-l}(T) \otimes B^{n^{\prime}-k+l}\left(T^{\prime}\right) .
$$

Duality then follows from the formula:

$$
\left(u \otimes u^{\prime}\right) \wedge\left(v \otimes v^{\prime}\right)= \pm(u \wedge v) \otimes\left(u^{\prime} \wedge v^{\prime}\right),
$$

where $u$ and $v$ are forms on $T$ and $u^{\prime}$ and $v^{\prime}$ are forms on $T^{\prime}$, pulled back to $T \times T^{\prime}$

This provides degrees of freedom for high order Whitney forms on products such as prisms and cubes, of possibly different degree in each direction. Remark that the construction can be used for cellular complexes whose cells are each a product of simplexes; the product need not be global.

Finally we want to consider finite element systems corresponding to the $h p$ setting, where polynomial degree is allowed to vary throughout the domain. We will require the following result:

Proposition 2.31 Suppose we have a cellular complex equipped with a finite element system $A$ having the extension property and the following property on cohomology. For each $T$ the following sequence is exact:

$$
0 \rightarrow A_{0}^{0}(T) \rightarrow A_{0}^{1}(A) \rightarrow \cdots \rightarrow A_{0}^{\operatorname{dim} T}(T) \rightarrow \mathbb{R} \rightarrow 0 .
$$

The second last arrow is integration. Then the finite element system is compatible.

- Proof: Suppose the required cohomology property (that is, without homogeneous boundary conditions) holds for all cells of dimension up to $l$. Then, following the proof of Theorem 2.16, one checks that the cohomology of $A^{\bullet}\left(\mathcal{T}^{\prime}\right)$ for subcomplexes $\mathcal{T}^{\prime}$ of $\mathcal{T}$ of dimension at most $l$ is given by the corresponding cochain complex. Letting $T$ be an $(l+1)$ dimensional cell we remark that $\partial T$ is such a subcomplex and we just do a variant of the proof of Proposition 2.20.

Suppose $\mathcal{T}$ is a cellular complex and that for each integer $p$ in some range, a compatible finite element system $A^{k, p}(T)$ has been chosen. We suppose that $A^{k, p}(T) \subseteq A^{k, p+1}(T)$. Choose now some function $p: \mathcal{T} \rightarrow \mathbb{N}$ with the property that if $T^{\prime} \subseteq T$ then $p\left(T^{\prime}\right) \leq p(T)$. Define:

$$
A^{k}(T)=\left\{u \in A^{k, p(T)}(T):\left.\forall T^{\prime} \subseteq T \quad u\right|_{T^{\prime}} \in A^{k, p\left(T^{\prime}\right)}\left(T^{\prime}\right) .\right.
$$

Proposition 2.32 These spaces constitute a compatible finite element system. 
- Proof: The surjectivity of restriction follows from the monotonicity of $p$, whereas the cohomological requirement follows from the preceding proposition using $A_{0}^{k}(T)=A_{0}^{k, p(T)}(T)$.

Now even if we have mirror-systems for each $A^{\bullet, p}(\cdot)$ this does not give a mirror-system for the constructed $A^{\bullet}(\cdot)$. Choosing the mirror $B^{\operatorname{dim} T-k}(T)$ for each $A_{0}^{k}(T)$ given by $p(T)$ does not guarantee that the restrictions map $B^{k}(T)$ to $B^{k}\left(T^{\prime}\right)$ when $T^{\prime} \subseteq T$, so the corresponding interpolator does not commute with the exterior derivative. Instead we shall introduce a generalization to any compatible finite element system of the projection based interpolation described for instance in [34].

We suppose a scalar product $a$ has been chosen for each $\Omega^{k}(T)$. This is similar to the choice of scalar products on $A^{k}(T)$ we required for the notion of local harmonicity. Let $\Psi^{k}$ be the map which to any compatible differential $k$-form $u$ associates with each $T \in \mathcal{T}$ the two linear forms $a(u, \cdot)$ on $\mathrm{d} A_{0}^{k-1}(T)$ and $a(\mathrm{~d} u, \cdot)$ on $\mathrm{d} A_{0}^{k}(T)$ when $k<\operatorname{dim} T$, and when $k=\operatorname{dim} T$, the linear form $a(u, \cdot)$ on $\mathrm{d}_{0}^{k-1}(T)$ and the number $\int u$ (which can be considered as a linear form on $\mathbb{R}$ ). We let $B^{k}(T)$ be the space $\mathrm{d} A_{0}^{k-1}(T) \oplus \mathrm{d} A_{0}^{k}(T)$ in the first case and $\mathrm{d} A_{0}^{k-1}(T) \oplus \mathbb{R}$ in the second. For $k>\operatorname{dim} T$ put $B^{k}(T)=0$. Thus, with the help of the scalar products, in each case an element of $\Omega^{k}(T)$ gives a linear form on $B^{k}(T)$.

Proposition 2.33 $\Psi^{k}$ induces an isomorphism $A^{k}(\mathcal{T}) \rightarrow \bigoplus_{T \in \mathcal{T}} B^{k}(T)^{\star}$.

- Proof: Injectivity follows from the uniqueness of Lemmas 2.7 and 2.8 applied in a recursive manner from cells of dimension $k$ and upwards. Then remark that we have equality of dimension by Propositions 2.15 and 2.20 .

As before we can deduce an interpolation operator $I^{k}$ which to a $k$ form $u$ associates the unique $v \in A^{k}(\mathcal{T})$ such that $\Psi^{k} v=\Psi^{k} u$.

Proposition 2.34 Interpolation thus defined commutes with the exterior derivative.

- Proof: Remark that for any compatible $k$-form $u$, if $\Psi^{k} u=0$ then $\Psi^{k+1} \mathrm{~d} u=0$. This is nothing but the property already used that locally harmonic forms have locally harmonic exterior derivative, in addition to Stokes theorem. From this remark the proposition follows.

We remark furthermore that there is an obvious isomorphism $\oplus_{T \in \mathcal{T}} A_{0}^{k}(T) \rightarrow$ $\oplus_{T \in \mathcal{T}} B^{k}(T)^{\star}$ determined on each cell by the chosen scalar products. Composing with the inverse of $\Psi^{k}$ gives an isomorphism $\oplus_{T \in \mathcal{T}} A_{0}^{k}(T) \rightarrow A^{k}(\mathcal{T})$. It is the map $E$ of Proposition 2.24.

As scalar products one can use for instance the $\mathrm{L}^{2}$ product associated with some Riemannian structure. In [35] fractional order Sobolev spaces are also used. Working with arbitrary scalar products $a$ allows also for scalar products obtained by transport from reference cells.

\section{Conclusion}

We indicate some directions in which to pursue the present work.

We reviewed homological algebra and tensor products for real vector spaces rather than modules over an arbitrary ring, since this leads to significant simplifications. However module theory might play a role in future investigations on finite elements, letting the rings be those of multivariate polynomials. This could shed light for instance on linear dependence relations in the natural spanning sets for high order Whitney 
forms. This is of consequence to the choice of bases, for which there are several suggestions [60] [39] [1] [37] [52] [6].

Another interesting line of investigation is finite elements for elasticity. Symmetric tensor fields have algebraic properties not covered by the present study. The linear elasticity complex [4] is related to but different from the De Rham complex.

Finally some non-linear wave equations such as the Yang-Mills and Einstein equations also have a rich algebraic structure that should be reflected in discretizations [28] [25] [26] [27] [23].

\section{References}

[1] M. Ainsworth AND J. Coyle: Hierarchic finite element bases on unstructured tetrahedral meshes; Int. J. Numer. Meth. Engng. 58 (2003) 2103-2130.

[2] F.P. Andriulli, K. Cools, H. Bagci, F. Olyslager, A. Buffa, S.H. Christiansen, E. Michielssen, Eric: A multiplicative Calderon preconditioner for the electric field integral equation; IEEE Trans. Antennas and Propagation 56 (2008), no. 8, part 1, 2398-2412.

[3] D.N. Arnold, R.S. Falk, R. Winther: Differential complexes and stability of finite element methods. I. The De Rham complex; Compatible spatial discretizations, 24-46, IMA Vol. Math. Appl., 142, Springer, New York, 2006.

[4] D.N. Arnold, R.S. Falk, R. Winther: Differential complexes and stability of finite element methods. II. The elasticity complex; Compatible spatial discretizations, 47-67, IMA Vol. Math. Appl., 142, Springer, New York, 2006.

[5] D.N. Arnold, R.S. Falk, R. Winther: Finite element exterior calculus, homological techniques, and applications; Acta Numerica, Vol. 15 , p. 1-155, 2006.

[6] D.N. Arnold, R.S. Falk, R. Winther: Geometric decompositions and local bases for spaces of finite element differential forms; 2008.

[7] I. BABUSKA: Error bounds for the finite element method; Numer. Math., Vol. 16, p. 322-333, 1971.

[8] I. Babuska, J. Osborn: Eigenvalue problems; Handbook of numerical analysis, Vol. II, 641-787, Handb. Numer. Anal., II, NorthHolland, Amsterdam, 1991.

[9] D. Boffi, F. Brezzi, L. Gastaldi: On the problem of spurious eigenvalues in the approximation of linear elliptic problems in mixed form; Math. Comp. 69 (2000), no. 229, 121-140.

[10] D. Boffi, P. Fernandes, L. Gastaldi, I. Perugia: Computational models of electromagnetic resonators: analysis of edge element approximation; SIAM J. Numer. Anal., Vol. 36, No. 4, p. $1264-1290$, 1999.

[11] A. Bossavit: Mixed finite elements and the complex of Whitney forms; The mathematics of finite elements and applications, VI (Uxbridge, 1987), p. 137-144, Academic Press, London, 1988.

[12] A. Bossavit: Discretization of electromagnetic problems: the "generalized finite differences" approach; Handbook of numerical analysis. Vol. XIII, 105-197, Handb. Numer. Anal., XIII, North-Holland, Amsterdam, 2005. 
[13] R. Botт, L.W. Tu: Differential forms in algebraic topology; Graduate texts in mathematics No. 82, Springer-Verlag, 1982.

[14] F. BrezzI: On the existence, uniqueness and approximation of saddle-point problems arising from Lagrangian multipliers; RAIRO Anal. Numér., Vol. 8, No. R-2, p. 129-151, 1974.

[15] F. Brezzi, J. Douglas, R. Durán, M. Fortin: Mixed finite elements for second order elliptic problems in three variables; Numer. Math. 51 (1987), no. 2, 237-250.

[16] F. Brezzi, M. Fortin: Mixed and hybrid finite element methods; Springer-Verlag, 1991.

[17] A. Buffa, S. H. Christiansen: The Electric Field Integral Equation on Lipschitz screens : definitions and numerical approximation; Numer. Math., Vol. 94, No. 2, p. 229 - 267, 2003.

[18] A. Buffa, S. H. Christiansen: A dual finite element complex on the barycentric refinement; Math. Comp., Vol. 76, p. 1743 - 1769, 2007.

[19] S. Caorsi, P. Fernandes, M. Raffetto: Spurious-free approximations of electromagnetic eigenproblems by means of Nédélec-type elements; Math. Model. Numer. Anal., Vol. 35, No. 2, p. 331 - 354, 2001.

[20] S. H. Christiansen: Discrete Fredholm properties and convergence estimates for the Electric Field Integral Equation; Math. Comp., Vol. 73, No. 245, p. 143 - 167, 2004.

[21] S. H. Christiansen: A div-curl lemma for edge elements; SIAM J. Numer. Anal.,Vol. 43, No. 1, p. 116 - 126, 2005.

[22] S. H. Christiansen: Stability of Hodge decompositions in finite element spaces of differential forms in arbitrary dimension; Numer. Math. 107 (2007), no. 1, 87-106.

[23] S. H. Christiansen: A construction of spaces of compatible differential forms on cellular complexes; Math. Models Methods Appl. Sci. 18 (2008), no. 5, 739-757.

[24] S. H. Christiansen: On the linearization of Regge calculus; University of Oslo, Department of Mathematics, Preprint Pure Mathematics, ISSN 0806-2439, No. 13, May 2008.

[25] S. H. Christiansen: Constraint preserving schemes for some gauge invariant wave equations; SIAM J. Sci. Comp., to appear.

[26] S. H. Christiansen, T. G. Halvorsen: Solving the Maxwell-KleinGordon equation in the Lattice Gauge Theory formalism; University of Oslo, Department of Mathematics, Preprint Pure Mathematics, ISSN 0806-2439, No. 17, June 2008.

[27] S. H. Christiansen, T. G. Halvorsen: Convergence of lattice gauge theory for Maxwell's equations; University of Oslo, Department of Mathematics, Preprint Pure Mathematics, ISSN 0806-2439, No. 21, September 2008.

[28] S. H. Christiansen, R. Winther: On constraint preservation in numerical simulations of Yang-Mills equations; SIAM J. Sci. Comp., Vol. 28, No. 1, p. 75 - 101, 2006.

[29] S. H. Christiansen, R. Winther: Smoothed projections in finite element exterior calculus; Math. Comp. 77 (2008), no. 262, 813-829. 
[30] P. G. Ciarlet: Basic error estimates for elliptic problems; Handbook of numerical analysis, Vol. II, 17-351, Handb. Numer. Anal., II, North-Holland, Amsterdam, 1991.

[31] P. Ciarlet, J. Zou: Fully discrete finite element approaches for time-dependent Maxwell's equations; Numer. Math. 82 (1999), no. 2, 193-219.

[32] G. Cohen, P. Joly, J.E. Roberts, N. Torduman: Higher order triangular finite elements with mass lumping for the wave equation; SIAM J. Numer. Anal. 38 (2001), no. 6, 2047-2078.

[33] M. Costabel, A. McIntosh: On Bogovskii and regularized Poincaré integral operators for De Rham complexes on Lipschitz domains; Université de Rennes, Prépublication 08-40, 2008.

[34] L. Demkowicz, A. Buffa: $H^{1}, H$ (curl) and $H$ (div)-conforming projection-based interpolation in three dimensions. Quasi-optimal pinterpolation estimates; Comput. Methods Appl. Mech. Engrg. 194 (2005), no. 2-5, 267-296.

[35] L. Demkowicz, J. KuRTz: Projection-based interpolation and automatic hp-adaptivity for finite element discretizations of elliptic and Maxwell problems; ESAIM Proceedings. Vol. 21 (2007) [Journées d'Analyse Fonctionnelle et Numérique en l'honneur de Michel Crouzeix], 1-15, ESAIM Proc., 21, EDP Sci., Les Ulis, 2007.

[36] S. I. Gelfand, Y. I. MANin: Methods of homological algebra (second edition); Springer Monographs in Mathematics, Springer-Verlag, 2003.

[37] J. Gopalakrishnan, L.E. Garca-Castillo, L.F. Demkowicz: Nédélec spaces in affine coordinates; Comput. Math. Appl. 49 (2005), no. 7-8, 1285-1294.

[38] R. Hiptmair: Canonical construction of finite elements; Math. Comp., Vol. 68, No. 228, p. 1325-1346, 1999.

[39] R. Hiptmain: High order Whitney forms; Prog. Electr. Res. (PIER) 32 (2001) 271-299.

[40] R. Hiptmain: Finite elements in computational electromagnetism; Acta Numerica, p. 237 - 339, Cambridge University Press, 2002.

[41] R. Hiptmain: Discrete compactness for p-version of tetrahedral edge elements; SAM, ETH, Research Report 2008-31, 2008.

[42] P. Joly: Variational methods for time-dependent wave propagation problems; Topics in computational wave propagation, 201-264, Lect. Notes Comput. Sci. Eng., 31, Springer, Berlin, 2003.

[43] F. KIKUCHI: On a discrete compactness property for the Nédélec finite elements; J. Fac. Sci. Univ. Tokyo, Sect. 1A Math., Vol. 36, p. 479 - 490, 1989.

[44] S. LAnG: Differential and Riemannian manifolds, Third edition; Springer-Verlag, New York, 1995.

[45] S. LAnG: Algebra. Revised third edition; Graduate Texts in Mathematics, Vol. 211, Springer, 2002.

[46] P. Monk: Analysis of a finite element method for Maxwell's equations; SIAM J. Numer. Anal. 29 (1992), no. 3, 714-729.

[47] P. Monk: An analysis of Nédélec's method for the spatial discretization of Maxwell's equations; J. Comput. Appl. Math. 47 (1993), no. 1, 101-121. 
[48] P. Monk: Finite Element Methods for Maxwell's Equations; Oxford University Press, 2003.

[49] J.-C. NÉDÉLEC: Mixed finite elements in $\mathbb{R}^{3}$; Numer. Math. 35 (1980), no. 3, 315-341.

[50] J.-C. NÉDÉLEC: A new family of mixed finite elements in $\mathbb{R}^{3}$; Numer. Math. 50 (1986), no. 1, 57-81.

[51] J. PASCIAK, P.S. VASSILEVSKI: Exact de Rham sequences of spaces defined on macro-elements in two and three spatial dimensions; SIAM J. Sci. Comput. 30 (2008), no. 5, 2427-2446.

[52] F. RAPETti: High order edge elements on simplicial meshes.M2AN Math. Model. Numer. Anal. 41 (2007), no. 6, 1001-1020.

[53] P.A. Raviart, J.-M. Thomas: A mixed finite element method for 2nd order elliptic problems; p. 292-315 in I. Galligani, E. Magenes (ed): Mathematical aspects of the finite element method ; Lecture Notes in Math., Vol. 606, Springer-Verlag, Berlin and New York, 1977.

[54] G. DeRham: Variétés différentiables. Formes, courants, formes harmoniques (Third edition); Publications de l'Institut de Mathématique de l'Université de Nancago, III. Actualités Scientifiques et Industrielles, No. 1222b. Hermann, Paris, 1973.

[55] J.E. Roberts, J.-M. Thomas: Mixed and hybrid methods; p. 523640, in P.G. Ciarlet, J.-L. Lions (eds.), "Handbook of numerical analysis, Vol. II, Finite element methods (Part 1)", North-Holland, 1991.

[56] J. SchÖBERL: A posteriori error estimates for Maxwell equations; Math. Comp. 77 (2008), no. 262, 633-649.

[57] J. SchöBerL, S. Zaglmayr: High order Nédélec elements with local complete sequence properties; COMPEL, Vol. 24, No. 2, p. $374-384$, 2005.

[58] G. Strang, G. J. Fix: An analysis of the finite element method. Prentice-Hall Series in Automatic Computation; Prentice-Hall, Inc., Englewood Cliffs, N. J., 1973.

[59] E. TONTI: Finite formulation of the electromagnetic field; PIER, Vol 32 , p. $1-44,2001$.

[60] J.P. WeBB: Hierarchal vector basis functions of arbitrary order for triangular and tetrahedral finite elements; IEEE Trans. Ant. Prop., 47 (1999), no. 8, 1244-1253.

[61] H. Whitney: Geometric integration theory; Princeton University Press, Princeton, N. J., 1957.

[62] K.S. YEE: Numerical solution of initial boundary value problems involving Maxwell's equations in isotropic media; IEEE Trans. Ant. Prop., p. 302 - 307, 1966.

[63] J. ZHAO: Analysis of finite element approximation for timedependent Maxwell problems; Math. Comp. 73 (2004), no. 247, 10891105 . 